Universidade de São Paulo

Instituto de Física

\title{
O Acoplamento Spin-Órbita no Estudo de Fases Topológicas em uma Rede Hexagonal de Baricentros
}

\section{Carlos Augusto Mera Acosta}

Orientador: Prof. Dr. Adalberto Fazzio

Dissertação apresentada ao Instituto de Física da Universidade de São Paulo para obtenção do título de Mestre em Ciências

Banca Examinadora:

Prof. Dr. Adalberto Fazzio (IF-USP)

Prof $^{\mathrm{a}}$. Dr ${ }^{\mathrm{a}}$. Helena Maria Petrilli (IF-USP)

Prof. Dr. Roberto Bechara Muniz (IF-UFF)

São Paulo

-2013- 


\section{FICHA CATALOGRÁFICA}

\section{Preparada pelo Serviço de Biblioteca e Informação}

do Instituto de Física da Universidade de São Paulo

\section{Acosta, Carlos Augusto Mera}

O acoplamento Spin-Órbita no estudo de fases topológicas em uma rede hexagonal de baricentros. - São Paulo, 2013.

Dissertação (Mestrado) - Universidade de São Paulo. Instituto de Física - Depto. de Física dos Materiais e Mecânica

Orientador: Prof. Dr. Adalberto Fazzio

Área de Concentração: Grupo SAMPA

Unitermos: 1. Física da Matéria Condensada; 2. Física do estado Sólido; 3. Estrutura dos Sólidos. 


\section{Agradecimentos}

Aos meus pais, Cesar Mera e Martha Acosta por serem uma fonte de força e esperanza.

À Gina Polo, minha esposa, por tanto amor, compreensão, carinho e apoio nesta etapa de nossas vidas.

Aos meus irmãos e minha sobrinha Amarilla.

Aos meus amigos que sempre, sem importar a distância, me transmitiram a melhor das energias.

Ao Professor Adalberto Fazzio por me permitir trabalhar no grupo SAMPA e pelas densas discussões acadêmicas.

Ao Matheus Paes Lima pelo apoio acadêmico incondicional, amizade e exemplo.

Ao Professor José Roque Da Silva pela colaboração e pertinentes sugestões.

Aos membros do grupo SAMPA e amigos do instituto de física pela amizade e apoio.

Às agências CNPq e o INCT de carbono pelo financiamento. 


\section{RESUMO}

Neste trabalho, foram estudadas as fases topológicas não-triviais presentes em sistemas formados pela deposição de átomos no grafeno. Encontramos que quando um átomo hibridiza fortemente com o grafeno, apresenta um momento magnético e um forte spin-órbita é possível a formação de uma "rede hexagonal de baricentros" que efetivamente gera uma estrutura de bandas característica de um efeito hall quântico anômalo. Especificamente, determinamos que o Ru satisfaz estas características. Quando este metal é depositado em uma configuração triangular no grafeno ocorrem picos na densidade de estados localizados no centro geométrico (baricentro) dos triângulos formados pelos Ru. Estes picos estão distribuídos de forma hexagonal e efetivamente geram uma estrutura de bandas que nas proximidades do nível de Fermi apresenta uma configuração de spin caraterística do efeito Hall quântico anômalo. Adicionalmente, encontramos que o sistema composto pela adsorção de Ba ou Sr no grafeno favorece a formação do efeito Hall quântico de spin. Neste sistema, o acoplamento spin-órbita (SOC) gera um gap mais de 1000 vezes maior ao predito no grafeno pristino. Para o estudo destes sistemas, implementamos no código SIESTA a aproximação "on-site" do acoplamento spinórbita via o formalismo dos pseudopotenciais relativísticos de norma conservada. Nossa implementação foi testada a partir do estudo de fenômenos já conhecidos: i) o "strong spin-splitting" gerado no grafeno pela adsorção de Au, ii) o efeito hall quântico de spin no poço quântico de $\mathrm{HgTe} / \mathrm{CdTe}$ e, iii) a formação de estados topológicos na superfície do $\mathrm{Bi}_{2} \mathrm{Se}_{3}$ e as fases magnéticas deste material com átomos de Mn adsorvidos.

Palavras chave: Acoplamento spin-órbita, Efeito Hall quântico de spin, Efeito Hall quântico anômalo, Isolantes topológicos, Grafeno. 


\section{ABSTRACT}

In this work, were studied the non-trivial topological phases present in systems formed by deposition of atoms in graphene. We found that when an atom hybridizes strongly with graphene, has a magnetic moment and a strong spin-orbit it is possible the formation of a "hexagonal network of barycentres"that effectively generates a structure band characteristic of a quantum anomalous Hall effect. Specifically, we determined that Ru satisfies these characteristics. When this metal is deposited in a triangular configuration in graphene, peaks occur in the density of localized states in the geometric center (centroid) of the triangles formed by $\mathrm{Ru}$. These peaks are distributed in a hexagonal structure and effectively generates a band structure that near the Fermi level has a spin configuration characteristic of the spin quantum Hall effect anomalous. Additionally, we found that the system composed by the adsorption of $\mathrm{Ba}$ or $\mathrm{Sr}$ in graphene, promotes the formation of spin quantum Hall effect. In this system, the spin-orbit coupling (SOC) generates a gap more than 1000 times grater that predicted in pristine graphene. To study these systems, we implemented in the code SIESTA the "on-site" approach of the spin-orbit coupling through the formalism of norm conserved relativistic pseudopotentials. Our implementation was tested from the study of phenomena already known: i) the "strong" spin-splitting generated in graphene by adsorption of $\mathrm{Au}$, ii) the quantum spin Hall effect in quantum well of $\mathrm{HgTe} / \mathrm{CdTe}$ and, iii) the formation of topological states in the surface of $\mathrm{Bi}_{2} \mathrm{Se}_{3}$ and the magnetic of this material with Mn atoms adsorbed.

Key words: Spin-orbit coupling, Quantum Spin Hall effect, Anomalous Quantum Hall effect, topological insulators, Graphene. 


\section{Conteúdo}

1 Introdução 1

2 O acoplamento spin-órbita 5

2.1 Equação de Dirac . . . . . . . . . . . . . . . . . 7

2.2 O Acoplamento Spin-Órbita na Teoria de Dirac . . . . . . . . . . 13

2.3 Equação de Dirac para um potencial central . . . . . . . . . . . 18

3 Estrutura eletrônica 20

3.1 Aproximação de Born Oppenheimer . . . . . . . . . . . . . . . 21

3.2 Teoria do funcional da densidade . . . . . . . . . . . . 25

3.3 Sistemas Periódicos . . . . . . . . . . . . . . . 31

3.4 Pseudopotencial de Norma Conservada . . . . . . . . . . . . . . . 34

4 Implementação do Acoplamento Spin-Órbita no Código SIESTA 39

4.1 Formalismo do Pseudopotencial Relativístico de Norma Conservada . 41

4.1.1 LCAO e aproximação "on-site" da interação spin-órbita . . . . 45 
4.1.2 Matriz de Densidade e Energia Total . . . . . . . . . . 47

4.1 .3 Forças ........................... 48

4.2 Código Siesta . . . . . . . . . . . . . . . . . . . . 49

5 Aplicações $\quad 51$

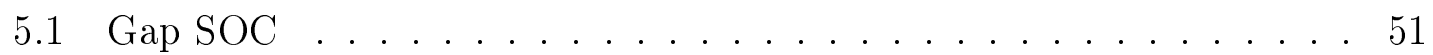

5.2 Efeito Hall Quântico de Spin . . . . . . . . . . . . . . . 55

5.2 .1 Fenomenologia do efeito QSH . . . . . . . . 58

5.2.2 Inversão de banda e estrutura eletrônica do HgTe . . . . . . . 63

5.2.3 Poços quântico em $\mathrm{HgTe} / \mathrm{CdTe}$... . . . . . . . . . . 64

5.3 Isolantes topológicos . . . . . . . . . . . . . 67

5.3.1 Estados topológicos em $\mathrm{Bi}_{2} \mathrm{Se}_{3} \ldots \ldots \ldots 71$

5.3.2 Ordem magnética no $\mathrm{Bi}_{2} \mathrm{Se}_{3}$ com átomos de $\mathrm{Mn}$ adsorvidos . . 73

5.4 "Strong Spin-Splitting" em Grafeno . . . . . . . . . . . . . 76

6 Fases topológicas em uma rede de "baricentros" com simetria $\begin{array}{ll}\text { hexagonal } & 80\end{array}$

6.1 Rede hexagonal de baricentros . . . . . . . . . . . . 83

6.1.1 Átomo de hidrogênio no baricentro ............ 98

$6.1 .2 \mathrm{STM} \ldots \ldots \ldots \ldots \ldots \ldots$

6.1.3 Rede triangular de $\mathrm{Ru} \ldots \ldots . \ldots 99$

6.1 .4 Rede retangular de $\mathrm{Ru} \ldots \ldots . . \ldots 101$

6.2 Efeito Hall quântico anômalo em baricentros . . . . . . . . . . . . . 102 
6.3 QSH em baricentros . . . . . . . . . . . . . 107

7 Conclusões

REFERÊNCIAS

114 


\section{Lista de Figuras}

2.1 Estrutura fina dos estados de menor energia no espectro do átomo de hidrogênio. . . . . . . . . . . . . . . . . 18

5.1 Estrutura de bandas do GaAs. . . . . . . . . . . . . 53

5.2 Esquema da mudança da estrutura de bandas no ponto $\Gamma$ em sistemas com simetria $T_{d} \ldots \ldots \ldots \ldots \ldots \ldots$

5.3 Variação do $\Delta_{0}$ em relação à força do SOC no GaAs. . . . . . . . 55

5.4 Comparação dos estados de borda em sistemas que apresentam o efeito QH e QSH. . . . . . . . . . . . . . 59

5.5 Formação do QSH a partir de duas copias do estado QH. . . . . . . . 61

5.6 Estrutura de bandas do HgTe e CdTe. . . . . . . . . . . . . . . . 64

5.7 Estados de interface no poço quântico de $\mathrm{HgTe} / \mathrm{CdTe} . . . . .66$

5.8 Comparação da superfície de Fermi para um isolante topológico forte e um isolante topológico fraco. . . . . . . . . . . . 68

5.9 Dispersão eletrônica entre dos pontos de superfície Kramers degene-

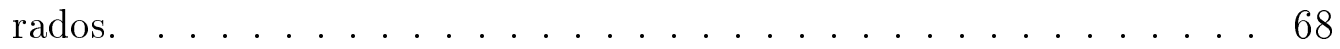


5.10 Inversão de banda no bulk de $\mathrm{Bi}_{2} \mathrm{Se}_{3} \ldots \ldots \ldots$. . . . . 70

5.11 Supercélula hexagonal de $\mathrm{Bi}_{2} \mathrm{Se}_{3} \ldots \ldots \ldots$. . . . . . . . . . 71

5.12 Estrutura de bandas para diferentes Ql's de $\mathrm{Bi}_{2} \mathrm{Se}_{3} \ldots \ldots . . . . .72$

5.13 Estrutura de bandas d3 4QL's $\mathrm{Bi}_{2} \mathrm{Se}_{3}$ com Mn adsorvido. . . . . . . 75

5.14 Figura esquemática dos sítios de adsorção no grafeno estudados. . . . 77

$5.15 \mathrm{Au}$ adsorvido na posição B em uma supercélula 4X4 de grafeno. . . . 78

5.16 Estrutura de bandas do Pt quando adsorvido na mono-vacância de uma supercélula 6 X6 de grafeno. . . . . . . . . . . . 79

6.1 Construção da rede de baricentros pela deposição de moléculas de

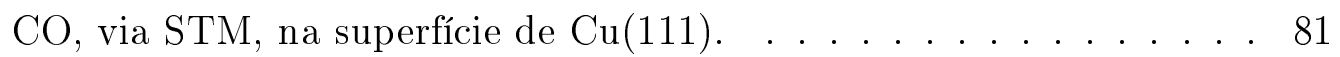

6.2 Estrutura de bandas do "bulk"de Rutênio. . . . . . . . . . . . . . . 84

6.3 Estrura de bandas do sistema formado pela adsorção do Ru em uma supercélula 5 X5 de grafeno. . . . . . . . . . . . . 85

6.4 Sequência da variação da estrutura de bandas. . . . . . . . . . . 86

6.5 Zona de Brillouin das supercéclulas 3X3, 4X4 e 5X5. . . . . . . . 89

6.6 Estrutura de bandas da supercélula 6X6 de grafeno pristino. . . . . . 90

6.7 LDOS por spin para cada cone da estrutura da debandas da

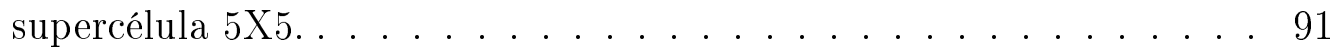

6.8 LDOS para os cones nas proximidades do nível de Fermi da estrutura de bandas da supercélula 4X4. . . . . . . . . . . . . . . . 92

6.9 Figura esquemática da molécula BMRu . . . . . . . . . . . . . . 93

6.10 Função de onda dos orbitais moleculares HOMO $\left(d_{x z}\right.$ e $\left.d_{y z}-\uparrow\right)$ e do LOMO $\left(d_{x z}\right.$ e $\left.d_{y z}-\downarrow\right)$ da molécula BMRu. . . . . . . . . . . . . 94 
6.11 Comparação do comportamento entre o HOMO(up dyz) e o LUMO(down dyz) em relação à variação da isosuperfície usada para "plotar" a função de

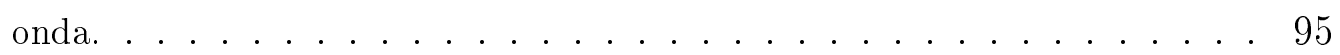

6.12 LDOS da famíla 4 X4 . . . . . . . . . . . . . . . 96

6.13 Átomo de hidrogênio no baricentro. . . . . . . . . . . . . . . 98

6.14 Imagen STM da rede hexagonal de baricentros para uma supercélula

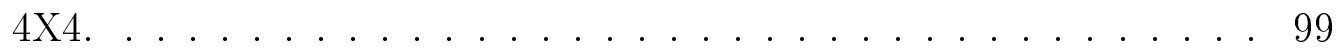

6.15 Estrutura de bandas do Ru depositados em configurações triangulares e hexagonais no grafeno. . . . . . . . . . . . . 100

6.16 Estrutura de bandas da rede retangular de Ru depositados em grafeno.102

6.17 (a)estrutura de banda do grafeno pristino com cones de Dirac centralizados nos pontos $K$ e $K^{\prime}$ no espaço recíproco. (b)Quando unicamente o potencial de troca é aplicado. (c) Quando só o SOC Rashba é aplicado, os estados de spin são misturados e a degenerescência de spin é levantada. (d) Um gap de "bulk" é aberto quando tanto o SOC Rashba e o campo de intercâmbio estão presentes. . . . . . . . . . . . . . . 103

6.18 Efeito HQA no Ru/Gr. . . . . . . . . . . . . . . . 104

6.19 Variação do Gap SOC em relação ao tamanho das supercélulas. . . . . . . 105

6.20 Variação da estrutura de bandas em relação à mudança da distância Ru-grafeno . . . . . . . . . . . . . . 106

6.21 Transferência do SOC do Ba ao grafeno. . . . . . . . . . . . . 109

6.22 Mudança da estrutura de bandas do Ba adsorvido no grafeno em relação à variação da intensidade do SOC do átomo de Ba. . . . . . . 110 


\section{Lista de Tabelas}

2.1 Soluções para os dois possíveis autovalores do operador $K \ldots \ldots$

2.2 Estados de menor momento angular total $j$ e número quântico SOC $\left(\kappa= \pm\left(j+\frac{1}{2}\right)\right) \ldots \ldots \ldots \ldots \ldots \ldots \ldots \ldots \ldots \ldots \ldots \ldots \ldots \ldots \ldots \ldots \ldots \ldots$

2.3 Estrutura fina: Estados de menor energia do átomo de hidrogênio. A energia é menor para menor $n$ e logo para menor $j \ldots \ldots 17$

5.1 Valores dos gap SOC em eV calculados usando o SIESTA-SOC comparados com os valores experimentais. . . . . . . . . . . 54

5.2 Valores dos gap SOC em eV calculados usando o SIESTA-SOC com diferentes tamanhos da base. . . . . . . . . . . . 55 


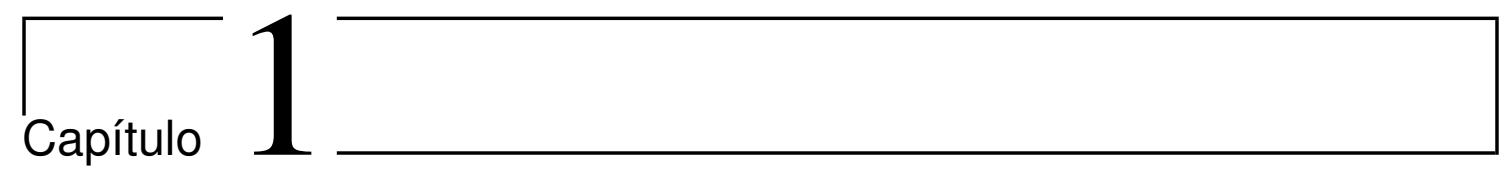

\section{Introdução}

O estado isolante, caracterizado por um gap de energia que separa as bandas eletrônicas totalmente preenchidas de um conjunto de bandas completamente vazias é um dos estados mais elementares da matéria. A física da matéria condensada do século XX revolucionou-se com a descoberta de um novo estado, caracterizado por "bulk" isolante com estados metálicos estáveis na borda robustos à desordem, denominado efeito Hall quântico (HQ). Este efeito é caraterizado por apresentar uma magneto-condutância transversal $\left(\sigma_{x y}\right)$ quantizada de uma maneira extremadamente precisa em número inteiro de $e^{2} / \hbar$, a despeito de termos um gap de energia. O efeito HQ presente em um gás de elétrons bidimensional a baixas temperaturas sob a ação de um forte campo magnético externo parecia violar a imagem na qual um sistema com gap de energia não apresenta transporte eletrônico. Este aparente paradoxo foi resolvido por Laughlin[1], observando que a função de onda tem um papel fundamental no transporte, ou seja, é uma propriedade da coerência de fase de funções de muitos corpos. A forma explícita de como esta fase está relacionada com $\sigma_{x y}$ foi posteriormente derivada por Thouless, Kohmoto, Nighting e Nijs (TKNN)[2], trazendo o conceito de topologia para a caraterização de estados isolantes na matéria. Enquanto os metais apresentam uma corrente "Ohmica", oriunda do desvio do equilíbrio na função de distribuição de Fermi-Dirac somente para estados próximos 
de nível de Fermi, a corrente Hall é uma corrente topológica que envolve todos os estados abaixo do nível de Fermi. A condutância Hall depende somente do número de estados de borda, os quais são unidirecionais devido à quebra de simetria de reversão-temporal que resulta da aplicação do campo magnético externo.

Na primeira década do século XXI a física da matéria condensada volta à exaltação pela descoberta de um análogo do QH denominado efeito Hall Quântico de Spin (QSH). Neste efeito, predito por Kane e Mele[3] o papel do campo magnético é fornecido pelo acoplamento spin-órbita, de forma que a simetria de reversão temporal é mantida. Esta singular caraterística resulta em um sistema isolante no "bulk" com um par de estados de bordas com spin propagando-se em direções opostas. Estes estados apresentam uma relação de dispersão linear - cone de Dirac, o qual mostra uma textura de spin com correntes de spin sem dissipação, suprimindo o retroespalhamento. Em consequência, os estados metálicos estáveis na borda são robustos à desordem sendo o mecanismo de "proteção" regido pela simetria de reversão temporal. O primeiro material que apresenta o QSH foi predito primeiramente por Zhang et al.[4], e observado experimentalmente por König et al.[5], no poço quântico (QW) de $\mathrm{HgTe} / \mathrm{CdTe}$. Entretanto, existem uma generalização 3D do QSH denominado isolante topológico (TI). O TI foi predito ocorrer na liga $\mathrm{Bi}_{1-x} \mathrm{Sb}_{x}$ para uma certa composição e, recentemente Hsieh et al.[6], através de Espectroscopia de Fotoemissão com Resolução Angular (ARPES) observaram os estados de superfície não-triviais nesse sistema. Esta classe de materiais, com propriedades exóticas, oferece um grande potencial para aplicações no campo da spintrônica. A descoberta de materiais que apresentam estados topológicos não triviais tornou-se um dos atuais focos de interesse dos físicos teóricos e experimentais da matéria condensada.

$\mathrm{Na}$ predição de novos materiais, seu entendimento e o estudo do comportamento em diferentes condições, as simulações computacionais possuem 
um papel fundamental dado que permitem o controle de condições, parâmetros e elementos. Contudo, para o estudo do efeito Hall quântico de spin via cálculos de primeiros princípios precisa-se do uso de um código computacional que leve em consideração o acoplamento spin-órbita. No contexto deste trabalho é importante salientar que do ponto de vista teórico, os métodos "ab initio" baseados na teoria do funcional da densidade (DFT) tem sido os mais utilizados para a caracterização dos estados topológicos, predição de outros materiais, efeitos de impurezas magnéticas, transporte eletrônico etc. Nosso grupo, dentro dos códigos computacionais de cálculos de primeiros princípios utiliza com frequência o código SIESTA, e tem feito implementações de forma a enriquecer o tratamento teórico. Como exemplo implementamos a $(i)$ correção de auto-interação (SIC) e o (ii) transporte eletrônico balístico via funções de Green fora do equilíbrio (Transampa-code), etc. Neste trabalho usando o formalismo de pseudopotenciais relativísticos de norma conservada implementamos no código SIESTA as correções relativísticas de primeira ordem, como o Acoplamento Spin-Órbita, fundamental para o entendimento das propriedades eletrônicas dos estados topológicos não-triviais. Esta implementação foi testada via o estudo dos seguintes fenômenos:

i. "Spin-splitting" no GaAs.

ii. QSH no poço quântico de HgTe/CdTe.

iii. A presença de estados metálicos protegidos pela simetria de reversão temporal na superfície do $\mathrm{TI} \mathrm{Bi}_{2} \mathrm{Se}_{3}$ e os efeitos da ordem magnética neste material com átomos de Mn adsorvidos na superfície.

iv. Efeitos do acoplamento spin-órbita na interação de metais de transição quando adsorvidos em grafeno.

Esta implementação foi usada para o estudo de fases topológicas presentes em sistemas formados pela deposição de átomos no grafeno. Motivados pela 
engenharia de superfícies no grafeno para a obtenção de estados associados com os efeitos: Hall quântico de spin e Hall quântico anômalo[64,75], procuramos átomos que permitam a existência destes efeitos quando adsorvido no grafeno. Entretanto este trabalho foi enfocado no estudo das fases topológicas de uma rede hexagonal de baricentros. Este sistema resulta da deposição de Ru em uma configuração triangular no grafeno, ou seja, esta singular configuração apresenta picos na densidade de estados localizados no centro geométrico dos triângulos formados pelos Ru. Estes centros geométricos (baricentros) formam uma rede com simetria hexagonal que de forma efetiva gera uma estrutura de bandas com quatro cones de Dirac. Nossa implementação permitiu inferir a classe topológica deste sistema.

A implementação da interação spin-órbita nos cálculos de estrutura eletrônica usando teoria do funcional da densidade exige o entendimento da origem deste fenômeno. No segundo capítulo deste trabalho é explicado como o termo associado ao acoplamento spin-órbita deriva naturalmente como uma consequência da teoria relativística da mecânica quântica de Dirac. No terceiro capítulo é apresentado o problema eletrônico e como a teoria do funcional da densidade permite resolvê-lo fazendo viável o estudo de sistemas compostos por muitos átomos com a inclusão dos pseudopotenciais de norma conservada. No capítulo quatro apresenta-se a forma de extrair o pseudopotencial de spin-órbita para cada valor do momento angular orbital, as aproximações feitas para a inclusão deste termo no formalismo da teoria do funcional da densidade. Na sequência, no capítulo cinco são desenvolvidos os estudos que verificam o bom funcionamento desta implementação. No capítulo seis, prediz-se a existência de uma rede hexagonal de baricentros gerada pela deposição de átomos no grafeno e estudam-se as possíveis fases topológicas deste sistema. Além do mais, mostra-se que existem metais (não reportados na literatura) que quando adsorvidos no grafeno estabilizam fases topológicas associadas com estados QSH. 


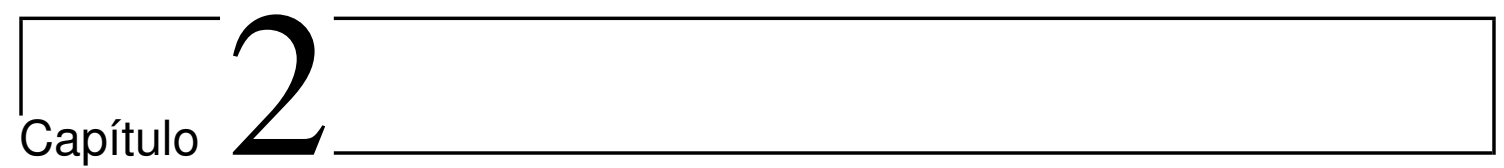

\section{O acoplamento spin-órbita}

Na teoria quântica de Schrödinger obtém-se uma descrição aproximada das propriedades dos sistemas atômicos. Embora esta descrição permita um entendimento profundo da matéria, não inclui os efeitos associados às correções relativísticas.

O espectro atômico é fortemente afetado pelo acoplamento spin-órbita (SOC)[8], o qual evidencia-se na estrutura fina do espectro de energia. O SOC é um efeito quântico relativístico sem análogo clássico associado à interação do momento magnético intrínseco do elétron com o momento angular orbital deste. Nos átomos, as correções relativísticas aumentam de relevância enquanto aumenta o número de orbitais atômicos ocupados ${ }^{1}$. Assim, alguns fenômenos que apresentam os sistemas que possuem átomos pesados, tal como o forte acoplamento spin-órbita, não podem ser entendidos na visão de Schrödinger da mecânica quântica.

Pode-se entender a origem do SOC mediante o estudo da teoria quântica

\footnotetext{
${ }^{1} \mathrm{O}$ tamanho do átomo de hidrogênio está dado pelo comprimento:
}

$$
a_{0}=\frac{\hbar}{\alpha m c} \Longrightarrow p \sim \alpha m c
$$

$\operatorname{com} \alpha=\frac{e^{2}}{4 \pi \epsilon_{0}} \frac{1}{\hbar c}$. As contribuições dos efeitos relativísticos na energia $E^{2}=p^{2} c^{2}+m^{2} c^{4}$ crescem com a massa, em quanto que o cociente $\frac{p c}{m c^{2}} \sim \alpha$ diminui. Assim, ao aumentar o tamanho do átomo as contribuições dos efeitos aumentam. 
relativística proposta por Paul Dirac, a qual em uma aproximação de ordem zero corresponde à descrição de Schrödinger da mecânica quântica. No entanto, introduz o SOC de forma natural como uma aproximação de primeira ordem. Consequentemente, na física atômica, a interação spin-órbita entra no hamiltoniano a partir de uma aproximação não-relativística da equação de Dirac. Esta aproximação dá origem ao termo conhecido como termo SO de Pauli:

$$
H_{S O}=-\frac{\hbar}{4 m_{0}^{2} c^{2}} \boldsymbol{\sigma} \cdot \hat{\boldsymbol{p}} \times\left(\nabla V_{0}\right)
$$

onde $\hbar$ é a constante de Planck, $m_{0}$ é a massa de um elétron livre, $c$ é a velocidade da luz, $\hat{\boldsymbol{p}}$ é o operador momento linear, $V_{0}$ é o potencial de Coulomb do núcleo atômico e $\boldsymbol{\sigma}=\left(\sigma_{x}, \sigma_{y}, \sigma_{z}\right)$ é o vetor das matrizes de Pauli. Desta forma, pode-se entender claramente como os graus de liberdade do spin afetam-se pelo SOC nos sistemas atômicos.

Em um sólido cristalino, o movimento dos elétrons está caracterizado pela energia de bandas $E_{n}(k)$ com índice de banda $n$ e vetor de onda $k$. Igualmente, neste caso o SOC apresenta um profundo efeito nas energias $E_{n}(k)$ associadas à estrutura de bandas. Por exemplo, a separação Zeeman de elétrons e buracos[9].

O SOC tem consequências importantes na estrutura de bandas dos semicondutores[8], por exemplo, no GaAs dá origem a uma quebra de degenerescência no topo da banda de valência cujos estados eletrônicos têm caráter $p$ (momento orbital angular $l=1$ ). Na presença do SOC os estados com momento angular total $j=3 / 2$ e $j=1 / 2$ estão separados em energia por um gap SOC $\Delta_{0}$. Embora, o exemplo anterior ilustra como o movimento orbital dos elétrons em um cristal é afetado pelo SOC, de forma geral não é óbvio com os efeitos do SOC evidenciam-se na estrutura eletrônica dos sólidos cristalinos.

Para entender o formalismo que permite levar em consideração o SOC 
no estudo da estrutura eletrônica de um sólido cristalino é necessário revisar os conceitos fundamentais associados à inclusão deste efeito na física atômica.

\subsection{Equação de Dirac}

O fato de que a equação de Schrödinger não descreva efeitos associados com correções relativísticas, ou seja, não seja invariante Lorentz, manifesta-se claramente na diferença da ordem das derivadas espaciais e temporais associadas às componentes do operador momento linear e à energia, respectivamente:

$$
\hat{H}_{0} \psi_{n}(\boldsymbol{r}, t)=\left[-\frac{\hbar}{2 m} \nabla^{2}+V(\boldsymbol{r})\right] \psi_{n}(\boldsymbol{r}, t)=i \hbar \frac{\partial}{\partial t} \psi_{n}(\boldsymbol{r}, t)
$$

sendo $\hat{H}_{0}$ o operador hamiltoniano de Schrödinger de um sistema na presença de um potencial $V(x)$ e $\psi_{n}(x, t)$ a função de onda.

Paul Dirac propôs uma equação na qual apareceram de forma equivalente as coordenadas espaço temporais e ao mesmo tempo apresentara o menor número de soluções possíveis para a relação energia-momento. Atualmente esta equação é conhecida como equação de Dirac. O operador hamiltoniano associado a uma partícula livre proposto por Dirac escreve-se como:

$$
\hat{H}=c \boldsymbol{\alpha} \cdot \hat{\boldsymbol{p}}+\beta m c^{2}=\sum_{i=1}^{3} c \alpha_{i} p^{i}+\beta m c^{2}
$$

onde $c$ é a velocidade da luz no vácuo, $m$ é a massa em repouso da partícula, $\hat{\boldsymbol{p}}$ o operador momento linear e $\alpha$ e $\beta$ são coeficientes.

Pode-se obter a forma dos coeficientes impondo que seja satisfeita a relação energia-momento relativística $E^{2}=p^{2} c^{2}+m^{2} c^{4}$. Assim, em termos do hamiltoniano 
de Dirac esta relação reescreve-se como:

$$
\hat{H}^{2}=c^{2} \sum_{i, k=1}^{3} \frac{1}{2}\left(\alpha_{i} \alpha_{k}+\alpha_{k} \alpha_{i}\right) p^{i} p^{k}+m c^{3} \sum_{i=1}^{3}\left(\alpha_{i} \beta+\beta \alpha_{i}\right) p^{i}+\beta^{2} m^{2} c^{4} .
$$

Levando em consideração que $i$ e $k$ são índices mudos e $p^{i}$ e $p^{k}$ comutam, as relações:

$$
\alpha_{i} \alpha_{k}+\alpha_{k} \alpha_{i}=2 \delta_{i k} ; \quad \alpha_{i} \beta+\beta \alpha_{i}=0 ; \quad \beta^{2}=1
$$

são condições suficientes e necessárias para que o hamiltoniano de Dirac esteja de acordo com a relação energia-momento relativística.

Para satisfazer estas condições os coeficientes $\alpha_{i}$ e $\beta$ devem ser matrizes. Como condição adicional as matrizem devem ser hermitianas para que o hamiltoniano também o seja.

As matrizes da mínima dimensão que satisfazem a relação $\alpha_{i} \alpha_{k}+\alpha_{k} \alpha_{i}=$ $2 \delta_{i k}$ são as matrizes de Pauli ${ }^{2}$, escritas como:

$$
\sigma_{x}=\left(\begin{array}{cc}
0 & 1 \\
1 & 0
\end{array}\right) ; \quad \sigma_{y}=\left(\begin{array}{cc}
0 & -i \\
i & 0
\end{array}\right) ; \quad \sigma_{z}=\left(\begin{array}{cc}
1 & 0 \\
0 & -1
\end{array}\right)
$$

No entanto, nesta dimensão $(2 \times 2)$ não existe nenhuma matriz que cumpra $\beta^{2}=1 \mathrm{e}$ $\beta \sigma_{i}+\sigma_{i} \beta=0$. Para satisfazer estas duas condições é necessário considerar matrizes de dimensão 4, sendo uma possível escolha:

$$
\alpha_{i}=\left(\begin{array}{cc}
0 & \sigma_{i} \\
\sigma_{i} & 0
\end{array}\right) ; \quad \beta=\left(\begin{array}{cc}
\mathcal{L} & 0 \\
0 & -\mathcal{L}
\end{array}\right)
$$

onde $\mathcal{L}$ é a matriz unitária $2 \times 2$. Na descrição de Dirac da mecânica quântica, a

\footnotetext{
${ }^{2}$ As matrizes de Pauli satisfazem:

$$
\sigma_{i} \sigma_{k}+\sigma_{k} \sigma_{i}=\left\{\sigma_{i}, \sigma_{k}\right\}=2 \delta_{i k} ; \quad\left[\sigma_{i}, \sigma_{j}\right]=2 i \epsilon_{i j k} \sigma_{k}
$$
}


equação de autovalores e autovetores para o hamiltoniano tem quatro componentes. Analogamente à equação de Schrödinger, pode-se escrever a equação de Dirac dependente do tempo da forma:

$$
\left(c \boldsymbol{\alpha} \cdot \hat{\boldsymbol{p}}+\beta m c^{2}\right) \psi(\boldsymbol{r}, t)=i \hbar \frac{\partial}{\partial t} \psi(\boldsymbol{r}, t),
$$

onde a função de onda tem quatro componentes:

$$
\psi(\boldsymbol{r}, t)=\left(\begin{array}{c}
\psi_{1}(\boldsymbol{r}, t) \\
\psi_{2}(\boldsymbol{r}, t) \\
\psi_{3}(\boldsymbol{r}, t) \\
\psi_{4}(\boldsymbol{r}, t)
\end{array}\right)
$$

Desta forma, a equação de Dirac proporciona uma descrição quântica que relaciona a função de onda com a energia de um sistema dentro da teoria relativística. Esta descrição denomina-se teoria quântica relativística.

Finalmente foram consideradas soluções de energia definida $\psi(\boldsymbol{r}, t)=$ $u(\boldsymbol{r}) e^{-i \frac{E t}{\hbar}}$ as quais permitem obter a equação de Dirac independente do tempo:

$$
\left(c \boldsymbol{\alpha} \cdot \hat{p}+\beta m c^{2}\right) u(\boldsymbol{r})=E u(\boldsymbol{r}) .
$$

\section{Forma Covariante da Equação de Dirac}

Para facilitar algumas demonstrações escreve-se a equação de Dirac numa notação covariante na qual definimos os quadrivetores associados à posição e ao 
momento ${ }^{3}$, como:

$$
x^{\mu}=(c t, \boldsymbol{r}) ; \quad p^{\mu}=\left(\frac{E}{c}, \boldsymbol{p}\right),
$$

de forma que o operador momento escreve-se nesta notação como:

$$
\hat{p}^{\mu}=i \hbar \nabla^{\mu}=i \hbar \frac{\partial}{\partial x_{\mu}}
$$

Usando estes elementos a equação de Dirac dependente do tempo expressa-se da forma:

$$
\left(\gamma_{\mu} \hat{p}^{\mu}-m c\right) \Psi(x, t)=0
$$

onde as componentes do operador $\gamma_{\mu}=\left(\gamma_{0}, \gamma_{1}, \gamma_{2}, \gamma_{3}, \gamma_{4}\right)$ são definidas em termos dos coeficientes $\alpha$ e $\beta$ :

$$
\gamma_{0}=\beta ; \quad \gamma_{i}=\beta \alpha_{i}
$$

\section{Correções Relativísticas}

Uma partícula na presença de um potencial $V(\boldsymbol{r})$ é observada em um estado caracterizado pela função de onda $\psi(\boldsymbol{r})=\left(\begin{array}{c}\phi \\ \chi\end{array}\right)$, onde $\phi$ e $\chi$ são vetores com duas componentes. Para este sistema a equação de Dirac independente do tempo pode ser escrita como:

$$
\left(\begin{array}{cc}
V(\boldsymbol{r})+m c^{2} & c \boldsymbol{\sigma} \cdot \hat{\boldsymbol{p}} \\
c \boldsymbol{\sigma} \cdot \hat{\boldsymbol{p}} & V(\boldsymbol{r})-m c^{2}
\end{array}\right)\left(\begin{array}{l}
\phi \\
\chi
\end{array}\right)=E\left(\begin{array}{l}
\phi \\
\chi
\end{array}\right) .
$$

\footnotetext{
${ }^{3} \mathrm{Na}$ forma contravariante escreve-se:
}

$$
x_{\mu}=(c t,-\boldsymbol{r}) ; \quad p_{\mu}=\left(\frac{E}{c},-\boldsymbol{p}\right)
$$


Fazendo $\epsilon=E-m c^{2}$ a equação de Dirac resulta em um sistema de equações:

$$
\begin{gathered}
(\boldsymbol{\sigma} \cdot \hat{\boldsymbol{p}}) \chi=\frac{1}{c}\{\epsilon-V(\boldsymbol{r})\} \phi, \\
(\boldsymbol{\sigma} \cdot \hat{\boldsymbol{p}}) \phi=\frac{1}{c}\left\{\epsilon-V(\boldsymbol{r})+2 m c^{2}\right\} \chi,
\end{gathered}
$$

Da equação (2.17) tem-se que:

$$
\chi=\frac{c}{\epsilon-V(\boldsymbol{r})+2 m c^{2}}(\boldsymbol{\sigma} \cdot \hat{\boldsymbol{p}}) \phi
$$

o que implica que se pode escrever a equação de Dirac em uma forma compacta:

$$
(\boldsymbol{\sigma} \cdot \hat{\boldsymbol{p}}) \frac{c}{\epsilon-V(\boldsymbol{r})+2 m c^{2}}(\boldsymbol{\sigma} \cdot \hat{\boldsymbol{p}}) \phi=\frac{1}{c}\left\{\epsilon-V(\boldsymbol{r})-m c^{2}\right\} \phi
$$

Considerando o limite não relativístico $\epsilon \ll m c^{2}$, pode-se escrever a expansão:

$$
\frac{1}{\epsilon-V(\boldsymbol{r})+2 m c^{2}}=\frac{1}{2 m c^{2}}\left\{\frac{1}{1+\frac{\epsilon-V(\boldsymbol{r})}{2 m c^{2}}}\right\} \sim \frac{1}{2 m c^{2}}\left\{1-\frac{\epsilon-V(\boldsymbol{r})}{2 m c^{2}}+\cdots\right\}
$$

De modo que em uma aproximação de ordem zero a equação de Dirac transforma-se na equação de Schrödinger independente do tempo $\hat{H}_{0} \phi=\left[\frac{\hat{p}^{2}}{2 m}+\right.$ $V(\boldsymbol{r})] \phi=\epsilon \phi$. O hamiltoniano do sistema em uma primeira ordem de aproximação reescreve-se em termos da expansão não relativística (2.20) como a soma do hamiltoniano de ordem zero $\hat{H}_{0}$ e as correções relativísticas de primeira ordem $\hat{H}_{1}$, da forma:

$$
\hat{H}=\hat{H}_{0}+\hat{H}_{1}
$$

onde as correções relativísticas satisfazem a relação:

$$
\hat{H}_{1} \phi=-(\boldsymbol{\sigma} \cdot \hat{\boldsymbol{p}}) \frac{\epsilon-V(\boldsymbol{r})}{4 m^{2} c^{2}}(\boldsymbol{\sigma} \cdot \hat{\boldsymbol{p}}) \phi
$$


'Porém, para potenciais centrais tem-se que:

$$
(\boldsymbol{\sigma} \cdot \hat{\boldsymbol{p}}) V(\boldsymbol{r})(\boldsymbol{\sigma} \cdot \hat{\boldsymbol{p}}) \phi=-\hbar^{2} V(\boldsymbol{r}) \boldsymbol{\nabla}^{2} \phi-\hbar^{2} \boldsymbol{\nabla} V(\boldsymbol{r}) \cdot \boldsymbol{\nabla} \phi-\frac{\hbar^{2}}{r} \frac{d V(\boldsymbol{r})}{d r} i \boldsymbol{\sigma} \cdot(\boldsymbol{r} \times \boldsymbol{\nabla}) \phi
$$

que pode ser reescrito da forma:

$$
(\boldsymbol{\sigma} \cdot \hat{\boldsymbol{p}}) V(\boldsymbol{r})(\boldsymbol{\sigma} \cdot \hat{\boldsymbol{p}}) \phi=\frac{1}{2}\left(\hat{\boldsymbol{p}}^{2} V(\boldsymbol{r})+V(\boldsymbol{r}) \hat{\boldsymbol{p}}^{2}\right) \phi+\frac{\hbar^{2}}{2} \nabla^{2} V(\boldsymbol{r}) \phi+\frac{2}{r} \frac{d V(\boldsymbol{r})}{d r} \boldsymbol{L} \cdot \boldsymbol{S} \phi
$$

Portanto, o termo de correção expressa-se como:

$$
\hat{H}_{1}=-\frac{\hat{\boldsymbol{p}}^{4}}{8 m^{3} c^{2}}+\frac{\hbar^{2}}{8 m^{2} c^{2}} \nabla^{2} V(\boldsymbol{r})+\frac{1}{2 m^{2} c^{2} r} \frac{d V(\boldsymbol{r})}{d r} \boldsymbol{L} \cdot \boldsymbol{S}
$$

por exemplo para o potencial Coulombiano gerado por um núcleo atômico as correções por efeitos relativísticos ficam como:

$$
\hat{H}_{1}=-\frac{\hat{\boldsymbol{p}}^{4}}{8 m^{3} c^{2}}+\frac{\pi \hbar^{2}}{2 m^{2} c^{2}}(Z \hbar c \alpha) \delta^{3}(\boldsymbol{r})+\frac{1}{2 m^{2} c^{2}} \frac{1}{r} \frac{d V(\boldsymbol{r})}{d r} \boldsymbol{L} \cdot \boldsymbol{S} .
$$

De acordo com esta expressão, classificam-se as correções relativísticas de primeira ordem como:

i. Correção à energia cinética

$$
\hat{T}_{r e l}=-\frac{p^{4}}{8 m^{3} c^{2}}
$$

ii. Acoplamento spin-órbita

$$
\hat{H}_{S O C}=\frac{1}{2 m^{2} c^{2}} \frac{1}{r} \frac{d V}{d r} \boldsymbol{L} \cdot \boldsymbol{S}
$$

iii. Termo de Darwin

$$
\hat{H}_{\text {Darwin }}=\frac{\pi \hbar^{2}}{2 m^{2} c^{2}}(Z \hbar c \alpha) \delta^{3}(r) .
$$




\subsection{O Acoplamento Spin-Órbita na Teoria de Dirac}

Na descrição de Dirac da mecânica quântica, ao calcular o comutador do hamiltoniano $\hat{H}=c \boldsymbol{\alpha} \cdot \hat{\boldsymbol{p}}+\beta m c^{2}$ e a projeção no eixo $z$ do momento angular orbital $\hat{\boldsymbol{L}}=\boldsymbol{r} \times \hat{\boldsymbol{p}}$, obtem-se:

$$
\left[\hat{H}, \hat{L}_{z}\right]=c \alpha_{i}\left[\hat{\boldsymbol{p}}_{i}, x\right] \hat{\boldsymbol{p}}_{y}-c \alpha_{i}\left[\hat{\boldsymbol{p}}_{i}, y\right] \hat{\boldsymbol{p}}_{x}=-i \hbar c\left(\alpha_{x} \hat{\boldsymbol{p}}_{y}-\alpha_{y} \hat{\boldsymbol{p}}_{x}\right)=-i \hbar c(\boldsymbol{\alpha} \times \hat{\boldsymbol{p}})_{z} .
$$

Na mecânica quântica relativística o operador momento angular de spin é definido em termos das matrizes de Pauli como um operador quadridimensional da forma:

$$
\sum_{i}=\left(\begin{array}{cc}
\sigma_{i} & 0 \\
0 & \sigma_{i}
\end{array}\right) ; \quad \hat{S}_{i}=\frac{\hbar}{2} \sum_{i}
$$

Consequentemente, satisfaz as regras de comutação de um operador de momento angular, ou seja, $\left[\hat{S}_{i}, \hat{S}_{j}\right]=i \hbar \epsilon_{i j k} \hat{S}_{k}$. Calculando o comutador da projeção deste operador no eixo z com o operador hamiltoniano de Dirac, obtem-se:

$$
\left[\hat{H}, \hat{S}_{z}\right]=\left[c \boldsymbol{\alpha} \cdot \hat{\boldsymbol{p}}+\beta m c^{2},-i \frac{\hbar}{4}\left(\alpha_{x} \alpha_{y}-\alpha_{y} \alpha_{x}\right)\right]=i \hbar c\left(\alpha_{x} \hat{\boldsymbol{p}}_{y}-\alpha_{y} \hat{\boldsymbol{p}}_{x}\right)=i \hbar c(\boldsymbol{\alpha} \times \hat{\boldsymbol{p}})_{z}
$$

Por conseguinte, tanto o operador momento angular orbital $\hat{\boldsymbol{L}}$ quanto o operador momento de spin $\hat{\boldsymbol{S}}$ não são bons números quânticos e, portanto, estes não são constantes de movimento. Porém, se definimos o operador $\hat{\boldsymbol{J}}=\hat{\boldsymbol{L}}+\hat{\boldsymbol{S}}$, o comutador com o hamiltoniano fica como:

$$
[\hat{H}, \hat{\boldsymbol{J}}]=0 \text {. }
$$


Assim, o momento angular que se conserva é o momento angular total:

$$
\hat{\boldsymbol{J}}=\boldsymbol{r} \times \hat{\boldsymbol{p}}+\frac{\hbar}{2} \sum
$$

A relação (2.33) implica em $\left[\hat{H}, \hat{J}^{2}\right]=0$ e $\left[\hat{H}, \hat{J}_{z}\right]=0$. No caso relativístico não se podem classificar os estados quânticos por meio de três operadores como no caso não relativístico, onde os estados quânticos ficam definidos pelos operadores $\hat{H}, \hat{J}^{2}$ e $\hat{J}_{z}$. Dado que o espaço é quadridimensional precisa-se de mais um operador que comute com os operadores $\hat{H}, \hat{J}^{2}$ e $\hat{J}_{z}$. Consequentemente, define-se o operador:

$$
\hat{K}=\beta \sum \cdot \hat{\boldsymbol{J}}-\frac{\hbar}{2} \beta=\beta\left(\sum \cdot \hat{\boldsymbol{L}}+\hbar \hat{\mathbf{1}}\right)
$$

que satisfaz as regras de comutação requeridas. O operador $K$ que é denominado "Operador do Acoplamento Spin-Órbita" e seus autovalores podem ser determinados a partir de:

$$
\hat{K}^{2}=\hat{L}^{2}+\hbar \sum \cdot \hat{\boldsymbol{L}}+\frac{3}{4} \hbar^{2}+\frac{1}{4} \hbar^{2}=\hat{J}^{2}+\frac{1}{4} \hbar^{2}
$$

que leva a estabelecer uma relação entre o quadrado do autovalor $\kappa$ do operador SOC e o autovalor $j$ do operador momento angular total:

$$
\hbar^{2} \kappa^{2}=\hbar^{2} j(j+1)+\frac{1}{4} \hbar^{2} \Longrightarrow \kappa^{2}=\left(j+\frac{1}{2}\right)^{2},
$$

Consequentemente, os autovalores do operador SOC ficam definidos pela expressão:

$$
\kappa= \pm\left(j+\frac{1}{2}\right)
$$

Tendo em vista que a descrição feita leva em consideração a presença do spin é necessário trabalhar na base acoplada de spin e momento angular. Delimitando a 
análise ao estudo de partículas de spin igual a $\frac{1}{2}$, a base conjunta expressa-se como:

$$
\chi_{j l}^{m_{j}}=\left\langle l, \frac{1}{2}, m_{j}-\frac{1}{2}, \frac{1}{2} \mid j, m_{j}\right\rangle Y_{l, m_{j}-\frac{1}{2}}(\boldsymbol{r})\left(\begin{array}{l}
1 \\
0
\end{array}\right)+\left\langle l, \frac{1}{2}, m_{j}+\frac{1}{2},-\frac{1}{2} \mid j, m_{j}\right\rangle Y_{l, m_{j}+\frac{1}{2}}(\boldsymbol{r})\left(\begin{array}{l}
0 \\
1
\end{array}\right)
$$

onde $\left\langle l, s, m_{l}, m_{s} \mid j, m_{j}\right\rangle$ denota os coeficientes de Clebch-Gordan associados ao acoplamento dos momentos angulares $\boldsymbol{L}$ e $\boldsymbol{S}$ com o momento angular total $\boldsymbol{J}^{4}$. Por conseguinte, a função de onda de quatro componentes que leva em consideração a parte radial pode ser escrita como:

$$
\Psi=\left(\begin{array}{cc}
g(\boldsymbol{r}) & \chi_{j l A}^{m} \\
i f(\boldsymbol{r}) & \chi_{j l B}^{m}
\end{array}\right) \Rightarrow \begin{gathered}
J^{2} \Psi=j(j+1) \hbar^{2} \Psi \\
J_{z} \Psi=m \hbar \Psi
\end{gathered}
$$

Impondo que esta função seja auto-estado do operador $\hat{K}=\beta(\Sigma \cdot \boldsymbol{L}+\hbar)$ e levando em consideração que:

$$
\boldsymbol{\sigma} \cdot \boldsymbol{L} \chi_{j l}^{m}=\hbar\left(j(j+1)-l(l+1)-\frac{3}{4}\right) \chi_{j l}^{m},
$$

os autovalores deste operador têm que satisfazer as relações:

$$
\begin{gathered}
-\kappa=\left(j(j+1)-l_{A}\left(l_{A}+1\right)+\frac{1}{4}\right) \\
\kappa=\left(j(j+1)-l_{B}\left(l_{B}+1\right)+\frac{1}{4}\right) .
\end{gathered}
$$

De modo que os dois possíveis valores do momento angular orbital $l_{A}$ e $l_{B}$ para os dois valores de $\kappa$ obedecem à tabela $2.1^{5}$. Deste modo, $l_{A}$ e $l_{B}$ podem ser considerados

${ }^{4} \mathrm{Na}$ base acoplada verifica-se:

$$
\hat{J}^{2} \chi_{j l}^{m_{j}}=j(j+1) \hbar^{2} \chi_{j l}^{m_{j}} ; \quad \hat{J}_{z} \chi_{j l}^{m_{j}}=m_{j} \hbar \chi_{j l}^{m_{j}} ; \quad \hat{L}^{2} \chi_{j l}^{m_{j}}=l(l+1) \hbar^{2} \chi_{j l}^{m_{j}}
$$

${ }^{5}$ Por exemplo para $l_{A}$ e $\kappa=j+\frac{1}{2}$, tem-se:

$$
-j-\frac{1}{2}=j^{2}+j-l_{A}\left(l_{A}+1\right)+\frac{1}{4}
$$


Tabela 2.1: Soluções para os dois possíveis autovalores do operador $K$

\begin{tabular}{ccc}
\hline \hline$\kappa$ & $l_{A}$ & $l_{B}$ \\
\hline \hline$j+\frac{1}{2}$ & $j+\frac{1}{2}$ & $j-\frac{1}{2}$ \\
$-\left(j+\frac{1}{2}\right)$ & $j-\frac{1}{2}$ & $j+\frac{1}{2}$ \\
\hline \hline
\end{tabular}

funções de $\kappa$ definidas pela tabela 2.1 , de forma que $\Psi$ seja autovetor do operador de acoplamento spin-órbita. Note-se que estas definições de $l_{A}$ e $l_{B}$ implicam em:

$$
\boldsymbol{\sigma} \cdot \boldsymbol{L} \chi_{j l_{A}}^{m}=-(1+\kappa) \hbar \chi_{j l_{A}}^{m} ; \quad \boldsymbol{\sigma} \cdot \boldsymbol{L} \chi_{j l_{B}}^{m}=-(1-\kappa) \hbar \chi_{j l_{B}}^{m}
$$

Pode-se expressar $\chi_{j l_{B}}^{m}$ em função de $\chi_{j l_{A}}^{m}$. Para isto é utilizado o operador $\frac{1}{r} \boldsymbol{\sigma} \cdot \boldsymbol{r}$, Tabela 2.2: Estados de menor momento angular total $j$ e número quântico SOC $\left(\kappa= \pm\left(j+\frac{1}{2}\right)\right)$.

\begin{tabular}{ccccc}
\hline$\kappa$ & $j$ & $l_{A}$ & $l_{B}$ & Estado \\
\hline-1 & $\frac{1}{2}$ & 0 & 1 & $\frac{s_{1}}{2}$ \\
1 & $\frac{1}{2}$ & 1 & 0 & $\frac{p_{1}}{2}$ \\
-2 & $\frac{3}{2}$ & 1 & 2 & $\frac{p_{3}}{2}$ \\
2 & $\frac{3}{2}$ & 2 & 1 & $\frac{d_{3}}{2}$ \\
-3 & $\frac{5}{2}$ & 2 & 3 & $\frac{d_{5}}{2}$ \\
3 & $\frac{5}{2}$ & 3 & 2 & $\frac{f_{5}}{2}$ \\
\hline
\end{tabular}

que deve cumprir:

$$
\frac{\boldsymbol{\sigma} \cdot \boldsymbol{r}}{r} \chi_{j l_{B}}^{m}=a \chi_{j l_{A}}^{m}+b \chi_{j l_{B}}^{m}
$$

Devido a que $\frac{\boldsymbol{\sigma} \cdot \boldsymbol{r}}{r}$ é um operador escalar, em princípio, deve conservar o momento angular total $j$. É possível mostrar que $a=-1$ e $b=0$, de forma que :

$$
\frac{\boldsymbol{\sigma} \cdot \boldsymbol{r}}{r} \chi_{j l_{A}}^{m}=-\chi_{j l_{B}}^{m}
$$

Assim, finalmente a função de onda na descrição de Dirac da mecânica quântica

$$
\begin{gathered}
\Rightarrow l_{A}\left(l_{A}+1\right)=j^{2}+2 j+\frac{3}{4}=\left(j+\frac{1}{2}\right)\left(j+\frac{3}{2}\right) \\
\Rightarrow l_{A}=j+\frac{1}{2}
\end{gathered}
$$


escreve-se como:

$$
\Psi=\left(\begin{array}{c}
g(\boldsymbol{r}) \chi_{j l A}^{m} \\
-f(\boldsymbol{r}) \frac{i}{r} \boldsymbol{\sigma} \cdot \boldsymbol{r} \chi_{j l A}^{m}
\end{array}\right)
$$

Esta função quando considerada na equação de Dirac com um potencial central associado à interação Coulombiana entre os elétron e núcleos proporciona os níveis de energia do espectro de estrutura fina. Estes são ilustrados na tabela 2.3 e esquematizados na figura 2.1 para o caso particular de um átomo de hidrogênio.

Tabela 2.3: Estrutura fina: Estados de menor energia do átomo de hidrogênio. A energia é menor para menor $n$ e logo para menor $j$.

\begin{tabular}{|c|c|c|c|c|c|}
\hline$n$ & $p=n-j-\frac{1}{2}$ & $j$ & $\kappa$ & $l_{A}$ & estado \\
\hline \hline 1 & 0 & $\frac{1}{2}$ & -1 & 0 & $1 \frac{s_{1}}{2}$ \\
\hline 2 & 1 & $\frac{1}{2}$ & -1 & 0 & $2 \frac{s_{1}}{2}$ \\
\hline 2 & 1 & $\frac{1}{2}$ & 1 & 1 & $2 \frac{p_{1}}{2}$ \\
\hline 2 & 0 & $\frac{3}{2}$ & -2 & 1 & $2 \frac{p_{3}}{2}$ \\
\hline 3 & 2 & $\frac{1}{2}$ & -1 & 0 & $3 \frac{s_{1}}{2}$ \\
\hline 3 & 2 & $\frac{1}{2}$ & 1 & 1 & $3 \frac{p_{1}}{2}$ \\
\hline 3 & 1 & $\frac{3}{2}$ & -2 & 1 & $3 \frac{p_{3}}{2}$ \\
\hline 3 & 1 & $\frac{3}{2}$ & 2 & 2 & $3 \frac{d_{3}}{2}$ \\
\hline 3 & 0 & $\frac{5}{2}$ & -3 & 2 & $3 \frac{d_{5}}{2}$ \\
\hline
\end{tabular}

Note-se que em um desenho pictórico do espectro de energia do átomo de hidrogênio, observa-se que o SOC induz uma correção na energia do estado com menor número quântico principal $n$. Embora neste átomo os efeitos do SOC consideram-se "fracos", há muito tempo é possível a observação experimental destes efeitos. Portanto, em condições realísticas, na estrutura eletrônica de átomos, moléculas e sólidos com forte SOC observam-se profundas mudanças. Para obter uma descrição teórica de acordo com a observação experimental é necessária a inclusão deste efeito relativístico. 


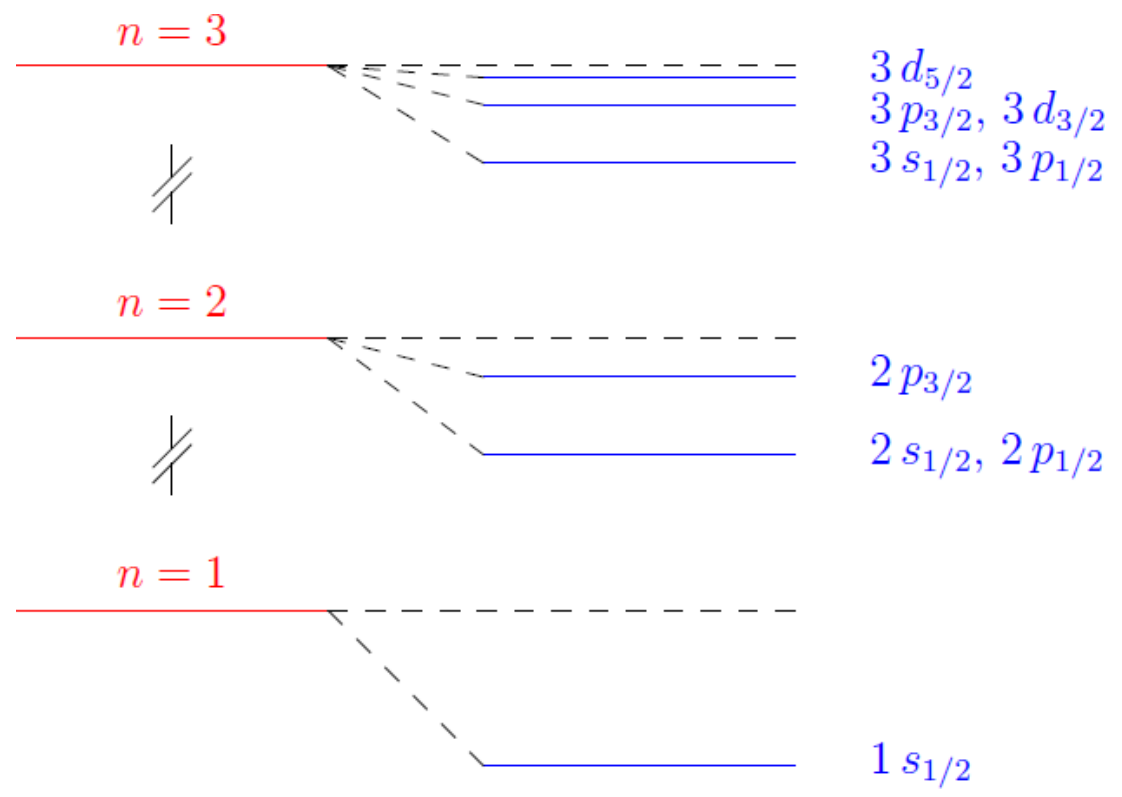

Figura 2.1: Estrutura fina dos estados de menor energia no espectro do átomo de hidrogênio. A separação de energia dos níveis associados ao numero quântico principal $n$ é maior que a quebra de degenerescência pela presença do SOC, referenciada na estrutura de bandas dos sólidos como gap SO.

\subsection{Equação de Dirac para um potencial central}

A equação de Dirac independente do tempo (2.10 ) para uma partícula sob a ação de um potencial central é escrita como:

$$
\left[c \boldsymbol{\alpha} \cdot \boldsymbol{p}+\beta m c^{2}+V(\boldsymbol{r})\right] \Psi=E \Psi
$$

de modo que, a comutação do hamiltoniano com o momento angular total $\boldsymbol{J}$ e operador SOC $K$ é mantida. Assim, o estado construído na seção anterior é adequado para descrever o sistema. Consequentemente, a equação de Dirac é reescrita em forma matricial da forma:

$$
\left(\begin{array}{cc}
V(\boldsymbol{r})+m c^{2}-E & c \boldsymbol{\alpha} \cdot \boldsymbol{p} \\
c \boldsymbol{\alpha} \cdot \boldsymbol{p} & V(\boldsymbol{r})-m c^{2}-E
\end{array}\right)\left(\begin{array}{c}
g(\boldsymbol{r}) \chi_{j l A}^{m} \\
-f(\boldsymbol{r}) \frac{i}{r} \boldsymbol{\sigma} \cdot \boldsymbol{r} \chi_{j l A}^{m}
\end{array}\right)=0 .
$$


A dependência entre os autovalores do operador SOC e o momento angular orbital permite reduzir este problema à resolução de um sistema de equações diferenciais:

$$
\begin{aligned}
& -\hbar c \frac{d f}{d r}+\frac{\hbar c(\kappa-1)}{r} f(\boldsymbol{r})=\left(E-m c^{2}-V(\boldsymbol{r})\right) g(\boldsymbol{r}) \\
& -\hbar c \frac{d g}{d r}+\frac{\hbar c(\kappa+1)}{r} g(\boldsymbol{r})=\left(E+m c^{2}-V(\boldsymbol{r})\right) f(\boldsymbol{r}),
\end{aligned}
$$

onde foi eliminado o fator global $\chi_{j l_{A}}^{m}$ operando pela esquerda com $\chi_{j l_{A}}^{m} \frac{\boldsymbol{\sigma} \cdot \boldsymbol{r}}{r}$. Podese escrever este sistema de equações de forma compacta definindo os operadores $F(\boldsymbol{r})=r f(\boldsymbol{r})$ e $G(\boldsymbol{r})=r g(\boldsymbol{r})$ de forma que,

$$
\frac{d f}{d r}=\frac{d}{d r} \frac{F(\boldsymbol{r})}{r}=\frac{1}{r} \frac{d F}{d r}-\frac{1}{r^{2}} F(\boldsymbol{r})
$$

onde $F(r)$ e $G(r)$ são denominadas solução maior e menor da equação radial de Dirac. Finalmente a equação radial de Dirac para uma partícula na presença de um potencial central em unidades atômicas expressa-se como:

$$
\begin{gathered}
\frac{d F}{d r}-\frac{\kappa}{r} F(r)+(\epsilon-V(r)) \lambda G(r)=0 \\
\frac{d G}{d r}+\frac{\kappa}{r} G(r)-\left(\frac{2}{\lambda^{2}}+\epsilon-V(r)\right) \lambda F(r)=0
\end{gathered}
$$

sendo $\epsilon=E-\lambda^{-2}, \operatorname{com} \lambda=\frac{1}{m c^{2}}$. 


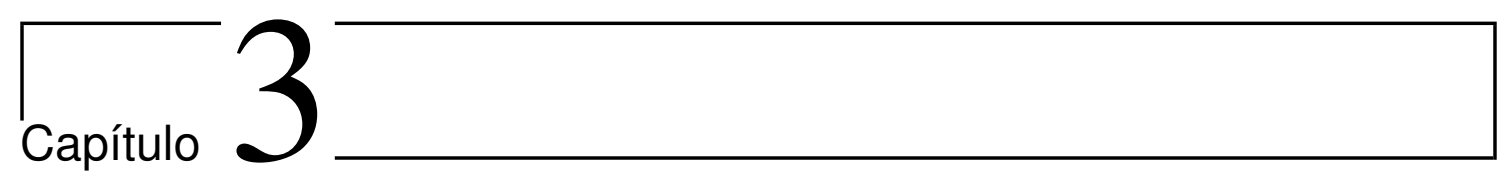

\section{Estrutura eletrônica}

As diferentes fases da matéria descobertas na natureza, tais como gasosa (moléculas) e condensada ("bulk"sólidos, superfícies, fios), podem ser descritas sem ambiguidade na mecânica quântica como um conjunto de $M$ núcleos atômicos e $N$ elétrons que interagem via forças eletrostáticas. Portanto, de acordo aos fundamentos da teoria quântica do estado sólido muitas das propriedades da matéria podem ser derivadas pela resolução da equação de Schrödinger independente do tempo:

$$
\hat{\mathcal{H}} \Psi_{n}(\boldsymbol{R}, \boldsymbol{r})=\varepsilon_{n} \Psi_{n}(\boldsymbol{R}, \boldsymbol{r}) .
$$

onde $\boldsymbol{R}=\left\{\boldsymbol{R}_{A}, \quad A=1,2, \ldots, P\right\}$ é o conjunto das $P$ coordenadas nucleares, $\boldsymbol{r}=\left\{\boldsymbol{r}_{\alpha}, \quad A=1,2, \ldots, P\right\}$ o conjunto das $N$ coordenadas eletrônicas e $\Psi_{n}(\boldsymbol{R}, \boldsymbol{r})$ a função de onda a qual depende das coordenadas atômicas e nucleares.

Nesta visão, o hamiltoniano $\hat{\mathcal{H}}$ leva em consideração as contribuições à energia do sistema $\varepsilon_{n}$ associadas aos operadores referentes à energia cinética dos núcleos $\hat{T}_{N}$, energia cinética dos elétrons $\hat{T}_{e}$, interação Coulombiana elétron-núcleo $\hat{V}_{N e}$, repulsão Coulombiana elétron-elétron $\hat{V}_{e}$ e interação eletrostática núcleo-núcleo $\hat{V}_{N}$. Nessa ordem, o hamiltoniano não relativístico associado ao sistema é escrito 
como:

$$
\hat{\mathcal{H}}=\hat{T}_{N}+\hat{T}_{e}+\hat{V}_{N e}+\hat{V}_{e}+\hat{V}_{N}
$$

Explicitando a forma dos operadores reescreve-se o hamiltoniano como:

$$
\begin{aligned}
\hat{\mathcal{H}}= & -\sum_{A=1}^{P} \frac{\hbar^{2}}{2 M_{A}} \nabla_{A}^{2}-\sum_{\alpha=1}^{N} \frac{\hbar^{2}}{2 m} \nabla_{\alpha}^{2}-\frac{e^{2}}{2} \sum_{A=1}^{P} \sum_{\alpha}^{N} \frac{Z_{A}}{\left|\boldsymbol{r}_{\alpha}-\boldsymbol{R}_{A}\right|} \\
& +\frac{e^{2}}{2} \sum_{\alpha=1}^{N} \sum_{\beta \neq \alpha}^{N} \frac{1}{\left|\boldsymbol{r}_{\alpha}-\boldsymbol{r}_{\beta}\right|}+\frac{e^{2}}{2} \sum_{A=1}^{P} \sum_{B \neq A}^{P} \frac{Z_{A} Z_{B}}{\left|\boldsymbol{R}_{A}-\boldsymbol{R}_{B}\right|},
\end{aligned}
$$

onde $Z_{A}$ e $M_{A}$ são a carga e a massa nucleares, respectivamente. A interação Coulombiana entre partículas de diferente tipo $\hat{V}_{N e}$ impede escrever a função de onda do sistema como o produto entre a função de onda eletrônica e a função de onda nuclear. Portanto, a obtenção das energias $\varepsilon_{n}$ é um problema que na prática é muito difícil de tratar dentro da mecânica quântica. De fato via tratamentos numéricos só tem solução exata para poucos sistemas. Consequentemente, precisa-se um enfoque que leve em consideração aproximações que permitam a resolução deste problema de muitos corpos interagentes sem perda de informação das propriedades eletrônicas, foco de interesse deste trabalho.

Neste capítulo apresenta-se um enfoque teórico e as aproximações envolvidas que permitem determinar as energias e funções de onda do hamiltoniano (3.1) mediante a solução da equação de Schrödinger.

\subsection{Aproximação de Born-Oppenheimer}

Tem sido demonstrado experimentalmente que o espectro molecular está separado nas regiões micro-ondas, infravermelho e ultravioleta que associam-se aos espectros rotacionais, vibracionais e eletrônico, respectivamente [11]. A visão teórica que consegue explicar satisfatoriamente este fato experimental é a chamada 
"Aproximação de Born-Oppenheimer".

Baseados na diferença das massas entre os elétrons e núcleos, Born e Oppenheimer propuseram o desacoplamento do movimento atômico e nuclear. Em uma visão clássica, o módulo da velocidade dos elétrons é muito maior que o módulo da velocidade dos núcleos, de forma que é possível considerar os núcleos como fixos em relação aos elétrons. Portanto, o termo de energia cinética nuclear $\hat{T}_{N}$ pode ser desprezado quando é comparado com os outros termos do hamiltoniano. Desta forma, o problema de muitos corpos interagentes reduz-se à resolução da equação de Schrödinger para um conjunto de elétrons interagentes na presença de um potencial externo devido aos núcleos.

No enfoque original, Born e Oppenheimer tratam a influência do movimento dos núcleos na função de onda eletrônica considerando um novo conjunto de variáveis nucleares $\boldsymbol{R}=\boldsymbol{R}_{0}+\boldsymbol{u}$, onde $\boldsymbol{u}$ representa o deslocamento dos núcleos em relação à posição de equilibro $\boldsymbol{R}_{0}$. Desta forma, obtêm-se o hamiltoniano como uma expansão em termos de $k=\left(m_{e} / M_{N}\right)^{\frac{1}{4}}$. Mantendo termos da expansão de quarta ordem em diante, não há mistura dos diferentes estados eletrônicos estacionários devido à interação com os núcleos. Portanto, em condições apropriadas ${ }^{1}$, os elétrons não sofrem transições entre estados estacionários.

Pode-se imaginar que os elétrons seguem instantaneamente o movimento dos núcleos ajustando sua função de onda à função de onda destes. Assim, para uma configuração particular nuclear $\boldsymbol{R}_{i}$, o potencial $V_{N e}$ representa uma interação eletrostática. De modo que, o sistema eletrônico sente a presença dos núcleos como um potencial externo $V_{\text {ext }}$. No entanto, neste podem ser levadas em consideração tanto a contribuição constante do operador interação Coulombiana núcleo-núcleo

\footnotetext{
${ }^{1}$ Além de ter que existir uma diferença de energia entre os possíveis estados estacionários do hamiltoniano eletrônico, esta diferença tem que ser maior às associadas a transições entre estados vibracionais do sistema. Desta forma, o efeito do movimento dos núcleos pode ser desprezado.

Em princípio a aproximação adiabática para metais não seria apropriada, dado que eles apresentam um gap nulo. No entanto, as excitações são confinadas a uma pequena região entorno da superfície de Fermi.
} 
quanto qualquer potencial externo de uma partícula. Isto permite escrever a equação de Schrödinger para o hamiltoniano eletrônico como:

$$
\hat{\mathcal{H}}_{e} \psi_{n}\left(\boldsymbol{R}_{i}, \boldsymbol{r}\right)=E_{n} \psi_{n}\left(\boldsymbol{R}_{i}, \boldsymbol{r}\right)
$$

com $\hat{\mathcal{H}}_{e}=\hat{T}_{e}+\hat{V}_{N e}+\hat{V}_{e}$. Desta forma, os elétrons permanecem sempre no mesmo estado estacionário do hamiltoniano eletrônico, o qual permite escrever uma expressão para a função de onda do sistema:

$$
\Psi(\boldsymbol{R}, \boldsymbol{r})=\sum_{m} \varphi_{m}(\boldsymbol{R}) \psi_{m}(\boldsymbol{R}, \boldsymbol{r})
$$

onde $\psi_{m}(\boldsymbol{R}, \boldsymbol{r})$ é a função de onda eletrônica para uma configuração nuclear particular e $\varphi_{m}(\boldsymbol{R})$ descreve a evolução do subsistema nuclear. Substituindo (3.5) em (3.1), obtém-se:

$$
\begin{gathered}
{\left[i \hbar \frac{\partial}{\partial t}+\sum_{A=1}^{P} \frac{\hbar^{2}}{2 M_{A}} \nabla_{A}^{2}-V_{N}-E_{n}(\boldsymbol{R})\right] \varphi_{n}(\boldsymbol{R}, t)=-\sum_{m} \sum_{A=1}^{P} \frac{\hbar^{2}}{2 M_{A}}\left\langle\psi_{n}\left|\nabla_{A}^{2}\right| \psi_{m}\right\rangle \varphi_{m}(\boldsymbol{R}, t)} \\
-2 \sum_{m} \sum_{A=1}^{P} \frac{\hbar^{2}}{2 M_{A}} \nabla_{A} \varphi_{m}(\boldsymbol{R}, t)\left\langle\psi_{n}\left|\nabla_{\boldsymbol{A}}\right| \psi_{m}\right\rangle .
\end{gathered}
$$

É clara a presença de termos não diagonais que acoplam os diferentes estados eletrônicos. Quando estes termos são desprezados não há acoplamento entre os estados estacionários associados ao hamiltoniano eletrônico, e a função de onda do sistema pode ser escrita como:

$$
\Psi(\boldsymbol{R}, \boldsymbol{r})=\varphi_{m}(\boldsymbol{R}) \psi_{m}(\boldsymbol{R}, \boldsymbol{r})
$$

o que implica que pode ser escrita uma equação para a contribuição eletrônica e outra para a evolução do subsistema composto pelos núcleos, sendo o hamiltoniano 
da contribuição eletrônica que obedece (3.4) dado por:

$$
\hat{\mathcal{H}}_{e}=-\sum_{\alpha=1}^{N} \frac{\hbar^{2}}{2 m} \nabla_{\alpha}^{2}-\frac{e^{2}}{2} \sum_{A=1}^{P} \sum_{\alpha}^{N} \frac{Z_{A}}{\left|\boldsymbol{r}_{\alpha}-\boldsymbol{R}_{A}\right|}+\frac{e^{2}}{2} \sum_{\alpha=1}^{N} \sum_{\beta \neq \alpha}^{N} \frac{1}{\left|\boldsymbol{r}_{\alpha}-\boldsymbol{r}_{\beta}\right|}
$$

e a equação que da conta da evolução nuclear escrita como:

$$
i \hbar \frac{\partial \varphi_{n}(\boldsymbol{R}, t)}{\partial t}=\left(-\sum_{A=1}^{P} \nabla_{A}^{2}+\widetilde{E}_{m}(\boldsymbol{R}, t)\right) \varphi_{n}(\boldsymbol{R}, t),
$$

onde:

$$
\widetilde{E}_{m}=E_{m}(\boldsymbol{R})+\sum_{A=1}^{P}\left\langle\varphi_{m}\left|\nabla_{A}^{2}\right| \varphi_{m}\right\rangle
$$

ou seja, o potencial efetivo sentido pelos núcleos está associado à energia total dos elétrons que se obtém da solução da equação (3.4).

Formalmente, a aproximação de Born Oppenheimer considera os termos de acoplamento diagonais como sendo nulos:

$$
\sum_{A=1}^{P}\left\langle\varphi_{m}\left|\nabla_{A}^{2}\right| \varphi_{m}\right\rangle=0
$$

de forma que a equação que descreve a evolução temporal dos núcleos reescreve-se como:

$$
i \hbar \frac{\partial \varphi_{n}(\boldsymbol{R}, t)}{\partial t}=\left(-\sum_{A=1}^{P} \nabla_{A}^{2}+E_{m}(\boldsymbol{R}, t)\right) \varphi_{n}(\boldsymbol{R}, t) .
$$

Desta forma, o movimento dos elétrons desacopla-se do movimento dos núcleos.

Inclusive na aproximação de Born-Oppenheimer o estudo das propriedades eletrônicas dos materiais é muito complicada já que é necessário determinar simultaneamente as funções de onda $\psi_{n}(\boldsymbol{R}, \boldsymbol{r})$ que dependem das coordenadas dos N elétrons interagentes para uma dada configuração nuclear. Entretanto, existem métodos apropriados dentro desta aproximação que permitem lidar com este problema, sendo a teoria do funcional da densidade a mais usada atualmente. Esta 
tem-se mostrado como a abordagem mais eficiente para o estudo das propriedades eletrônicas e estruturais do estado fundamental de sólidos cristalinos e moléculas.

\subsection{Teoria do Funcional da Densidade}

A procura de um formalismo que permita o estudo de sistemas descritos pelo hamiltoniano (3.8), iniciou com a proposta de Tomas e Fermi[12] de usar a densidade total como variável fundamental no cálculo da energia de um sistema atômico. Embora a abordagem original apresentava dificuldades para ser aplicada a sistemas ligados, esta estabeleceu as bases para desenvolvimentos posteriores que deram origem a o que hoje é conhecido como Teoria do Funcional da Densidade (DFT).

Formalmente a DFT permite escrever a energia de N elétrons interagentes na presença de um potencial externo como um funcional da densidade eletrônica total. Em outras palavras, tendo em vista que a densidade é um campo escalar no espaço tridimensional, a DFT compacta o problema eletrônico numa equação de somente três variáveis associadas à densidade eletrônica, ao invés de $3 \mathrm{~N}$ variáveis da função de onda. Assim, a energia total expressa-se como:

$$
E[\rho]=T[\rho]+U[\rho]+\int d r^{3} r \rho(\boldsymbol{r}) V_{e x t}(\boldsymbol{r})
$$

onde o primeiro termo está associado com a contribuição da energia cinética, o segundo corresponde à interação Coulombiana elétron-elétron e o último é a contribuição à energia associada ao potencial externo o qual leva em conta a interação elétron-núcleo além de outros potenciais de uma partícula. 
A interação Coulombiana

$$
U[\rho]=\sum_{i \neq j} \int d^{3} r_{1} \ldots \int d^{3} r_{N} \frac{\Psi_{0}^{*}\left(\boldsymbol{r}_{1}, \ldots \boldsymbol{r}_{N}\right) \Psi_{0}\left(\boldsymbol{r}_{1}, \ldots \boldsymbol{r}_{N}\right)}{2\left|\boldsymbol{r}_{i}-\boldsymbol{r}_{j}\right|}
$$

representa uma dificuldade na determinação dos estados e energias do hamiltoniano eletrônico. Entretanto, este termo pode ser separado em duas contribuições: a parte clássica da interação Coulombiana e os efeitos puramente quânticos desta:

$$
U[\rho]=\frac{1}{2}\left[\int d^{3} r_{1} \int d^{3} r_{2} \frac{\rho\left(\boldsymbol{r}_{1}\right) \rho\left(\boldsymbol{r}_{2}\right)}{\left|\boldsymbol{r}_{1}-\boldsymbol{r}_{2}\right|}+\int d^{3} r_{1} \int d^{3} r_{2} \frac{f_{2}\left(\boldsymbol{r}_{1} \boldsymbol{r}_{2}\right)}{\left|\boldsymbol{r}_{1}-\boldsymbol{r}_{2}\right|}\right]
$$

onde:

$$
f_{2}\left(\boldsymbol{r}_{1}, \boldsymbol{r}_{2}\right)=\gamma\left(\boldsymbol{r}_{1}, \boldsymbol{r}_{2}\right)-\rho\left(\boldsymbol{r}_{1}\right) \rho\left(\boldsymbol{r}_{2}\right)
$$

sendo $\gamma\left(\boldsymbol{r}_{1}, \boldsymbol{r}_{2}\right)$ a matriz de densidade reduzida ${ }^{2}$. Desta forma é possível identificar na expressão (3.15) a interação Coulombiana puramente clássica, também chamada termo de Hartree:

$$
U_{\text {Hartree }}[\rho]=\frac{1}{2} \int d^{3} r_{1} \int d^{3} r_{2} \frac{\rho\left(\boldsymbol{r}_{1}\right) \rho\left(\boldsymbol{r}_{2}\right)}{\left|\boldsymbol{r}_{1}-\boldsymbol{r}_{2}\right|} .
$$

A contribuição que provem dos efeitos quânticos é chamada energia de troca e sua forma exata não é conhecida. No entanto, podemos expressar a energia de troca como a diferença entre o funcional associado à interação Coulombiana $U[\rho]$ e à parte clássica desta $U_{\text {Hartree }}[\rho]$.

Por outra parte, os efeitos associados com a correlação eletrônica podem ser incluídos fazendo a diferença entre o funcional da energia cinética do sistema $T[\rho]$ e o funcional de energia cinética de um sistema de $N$ elétrons não interagentes

\footnotetext{
${ }^{2} \mathrm{~A}$ matriz de densidade reduzida expressa-se como:

$$
\gamma\left(\boldsymbol{r}_{1} \boldsymbol{r}_{2}\right)=N(N-1) \int \ldots \int d^{3} r_{3} \ldots d^{3} r_{N} \Psi_{0}^{*}\left(\boldsymbol{r}_{1}, \ldots \boldsymbol{r}_{N}\right) \Psi_{0}\left(\boldsymbol{r}_{1}, \ldots \boldsymbol{r}_{N}\right)
$$
}


$T_{s}[\rho]$.

Comumente os efeitos puramente quânticos da interação Coulombiana elétron-elétron e da correlação eletrônica evidenciados na energia cinética são escritos no funcional:

$$
E_{x c}[\rho]=U[\rho]-U_{\text {Hartree }}[\rho]+T[\rho]-T_{s}[\rho],
$$

o qual conhece-se como funcional de troca e correlação (exchange e correlation), na prática este será o único termo aproximado. Assim, o funcional energia eletrônica total reescreve-se da forma:

$$
E[\rho]=T_{s}[\rho]+E_{\text {ext }}[\rho]+E_{\text {Hartree }}[\rho]+E_{x c}[\rho],
$$

onde $T_{s}[\rho]$ é o funcional energia cinética do sistema eletrônico sem levar em conta correlação, $E_{\text {ext }}[\rho]$ a contribuição à energia associada a um potencial externo de uma partícula no qual se consideram as contribuições associadas à interação Coulombiana elétron-núcleo, $E_{\text {Hartree }}$ é o funcional associado à parte clássica da interação Coulombiana elétron-elétron e $E_{x c}[\rho]$ é o funcional que leva em conta as contribuições da correlação eletrônica da energia cinética (energia de correlação) e os efeitos quânticos da repulsão Coulombiana elétron-elétron (energia de troca), conhecido como funcional de troca e correlação.

Embora a descrição anterior especifica a forma na qual escreve-se o funcional de energia eletrônica, não evidencia a forma na qual a DFT compacta o problema eletrônico. A solução exata para o estado fundamental de um sistema de muitos corpos usando como objeto principal a densidade eletrônica total $\rho(\boldsymbol{r})$ foi dada por Hohenberg e Kohn[13]. Os teoremas que levam seus nomes constituem o nascimento da DFT moderna. 


\section{Formulação de Hohenberg e Kohn da DFT}

A formulação da teoria do funcional da densidade proposta por Hohenberg-Kohn[13], afirma que o valor esperado $\langle A\rangle$ de qualquer operador $\hat{A}$ é um funcional único da densidade eletrônica do estado fundamental $\rho(\boldsymbol{r})$, o qual significa que o valor de $\langle A\rangle$ depende do valor da densidade em todos os pontos. Como será mostrado, isto implica que, em princípio, todas as propriedades físicas do sistema podem ser determinadas com somente o conhecimento da densidade eletrônica do estado fundamental. Está ideia é condensada em dois teoremas:

\section{A densidade eletrônica total como variável básica}

A resolução da equação de Schrödinger para o hamiltoniano $\hat{\mathcal{H}}_{e}$ permite determinar todos os autovetores de muitos corpos $\psi_{n}\left(\boldsymbol{R}_{i}, \boldsymbol{r}\right)$. Desta forma, pode-se calcular o valor esperado de qualquer operador. Portanto, o hamiltoniano determina o valor esperado de qualquer observável, em particular, da densidade eletrônica do estado fundamental.

Tendo em vista que o operador energia cinética e a interação Coulombiana são universais ${ }^{3}$, o hamiltoniano é caraterizado unicamente pelo potencial externo $V_{\text {ext }}[\rho]$. Em consequência, o potencial externo define univocamente o valor esperado da densidade eletrônica do estado fundamental. Assim o primeiro teorema expressase como:

"A densidade eletrônica total do estado fundamental $\rho(\boldsymbol{r})$ de um sistema ligado de elétrons interagentes em algum potencial externo $V(\boldsymbol{r})$ determina univocamente este potencial, a menos de uma constante aditiva."

\footnotetext{
${ }^{3} \mathrm{O}$ termo "universal" faz referência a que os operadores são os mesmos para todos os sistemas de $\mathrm{N}$ elétrons interagentes não relativísticos.
} 


\section{Princípio variacional}

"A energia do estado fundamental é minima para a densidade $\rho(\boldsymbol{r})$ exata".

Hohenberg e Kohn mostraram que para o estudo das propriedades de um sistema de $\mathrm{N}$ elétrons interagentes no estado fundamental pode ser usada somente a densidade eletrônica total como a variável fundamental. Assim mediante a minimização da energia em relação à densidade pode ser determinada a energia e a densidade do estado fundamental. No entanto, não explicitaram a forma na qual aplica-se este princípio.

\section{Equações de Khon-Sham}

Um ano após a publicação dos trabalhos de Hohenberg e Kohn que deram nascimento à DFT, Kohn e Sham[14] construíram um formalismo para aplicação prática desta. O esquema para minimizar a energia do estado fundamental proposta por Kohn-Sham está baseado em considerar um sistema fictício de N elétrons não interagentes, de forma que o potencial efetivo que atua sob este sistema eletrônico gere uma densidade eletrônica exatamente igual à densidade eletrônica do sistema real. A energia do estado fundamental do sistema de $N$ elétrons não interagentes escreve-se como:

$$
E_{s}[\rho]=T_{s}[\rho]+\int d^{3} r \rho(\boldsymbol{r}) V_{e f f}(\boldsymbol{r})
$$

Mantendo o número de elétrons $N=\int d^{3} r \rho(\boldsymbol{r})$ constante, pode-se minimizar a energia deste sistema fazendo uso do princípio variacional:

$$
\frac{\delta}{\delta \rho}\left[E_{s}[\rho]-\mu\left(\int d^{3} r \rho(\boldsymbol{r})-N\right)\right]
$$


que leva à seguinte equação:

$$
\frac{\delta T_{s}[\rho]}{\delta \rho}+V_{e f f}(\boldsymbol{r}-\mu)=0
$$

A energia do sistema real $E[\rho]$ é minimizada com o vínculo $N=\int d^{3} r \rho(\boldsymbol{r})$ via o princípio variacional. Portanto, obtem-se uma equação análoga à equação (3.19):

$$
\frac{\delta}{\delta \rho}\left[E[\rho]-\mu\left(\int d^{3} r \rho(\boldsymbol{r})-N\right)\right]
$$

que implica em:

$$
\frac{\delta T_{s}[\rho]}{\delta \rho}+V_{\text {Hartree }}(\boldsymbol{r})+V_{\text {ext }}(\boldsymbol{r})+\frac{E_{x c}[\rho]}{\delta \rho}-\mu=0
$$

No esquema do Kohn-Sham[14], a densidade eletrônica do sistema não interagente deve ser igual à densidade do sistema de elétrons interagentes indicando uma equivalência entre as equações (3.20) e (3.22) o qual implica numa expressão para o potencial efetivo do sistema não interagente:

$$
V_{\text {eff }}(\boldsymbol{r})=V_{\text {Hartree }}(\boldsymbol{r})+V_{\text {ext }}(\boldsymbol{r})+\frac{\delta E_{x c}[\rho]}{\delta \rho}
$$

A determinação da densidade eletrônica do sistema de $N$ elétrons interagentes reduzse à resolução da equação de um sistema de $N$ elétrons não interagentes sob ação de um potencial efetivo $V_{\text {eff }}$. Esta equação é conhecida como equação de Kohn-Sham e comumente expressa-se da forma:

$$
\left[-\frac{1}{2} \nabla^{2}-V_{e f f}(\boldsymbol{r})\right] \psi_{\alpha}^{K S}(\boldsymbol{r})=\epsilon_{\alpha}^{K S} \psi_{\alpha}^{K S}(\boldsymbol{r})
$$

onde se introduz a contribuição associada ao funcional da energia cinética do sistema não interagente $T_{s}[\rho]$ em termos das denominadas funções de onda de Kohn-Sham 
$\psi_{\alpha}^{K S}(\boldsymbol{r})$, como:

$$
T_{s}[\rho]=\sum_{\alpha=1}^{N} \psi_{\alpha}^{K S *}(\boldsymbol{r})\left[-\frac{1}{2} \nabla^{2}\right] \psi_{\alpha}^{K S}(\boldsymbol{r})
$$

Nesta visão do problema, a densidade eletrônica é dada por:

$$
\rho(\boldsymbol{r})=\sum_{\alpha=1}^{N} \psi_{\alpha}^{K S *}(\boldsymbol{r}) \psi_{\alpha}^{K S}(\boldsymbol{r})
$$

a qual junto com as energia de Kohn-Sham $\epsilon_{\alpha}^{K S}$ são obtidas pela resolução autoconsistente da equação de KS. Note-se que as energias de Khon-Sham são apenas os autovalores desta equação auxiliar de uma partícula construída de forma que forneça a densidade eletrônica exata do estado fundamental.

A densidade do estado fundamental deve satisfazer a simetria do sistema estudado. Consequentemente, a equação de KS deve ser resolvida impondo a simetria do sistema. Entretanto, a simetria translacional dos sólidos cristalinos permite escrever a função de onda de KS como uma expansão de um conjunto completo de funções que satisfazem as condições periódicas do cristal.

\subsection{Sistemas Periódicos}

Um dos conceitos fundamentais na descrição de um sólido cristalino é a rede de Bravais, que especifica como as unidades básicas que o compõem (átomos, grupos de átomos ou moléculas) repetem-se periodicamente ao longo do cristal como um conjunto de pontos no espaço tal que exista simetria translacional. Os vetores de translação $\left\{\boldsymbol{R}_{n}\right\}$ da rede de Bravais definem-se de maneira que quando uma operação de translação $t\left\{\boldsymbol{R}_{n}\right\}$ aplica-se, o sistema resultante é indistinguível do sistema original. Consequentemente, o potencial gerado pelos átomos que conformam um determinado cristal deve obedecer às condições periódicas impostas pela simetria translacional. De modo que $V\left(\boldsymbol{r}+\boldsymbol{R}_{n}\right)=V(\boldsymbol{r})$. 
Espera-se que a densidade eletrônica do estado fundamental, resultante da solução da equação de KS, também seja periódica. Portanto, nas equações de KS o potencial efetivo também será periódico.

Neste ponto, o teorema de Bloch poderia ser adicionado, agregando um número quântico $\boldsymbol{k}$, e desta forma as funções de onda de KS obedecem a relação:

$$
\varphi_{\alpha k}^{K S}\left(\boldsymbol{r}+\boldsymbol{R}_{n}\right)=e^{i \boldsymbol{k} \cdot \boldsymbol{R}_{n}} \varphi_{\alpha k}^{K S}(\boldsymbol{r})
$$

e as equações de KS são dadas por:

$$
\left[-\frac{1}{2} \nabla^{2}+V_{e f f}(\boldsymbol{r})\right] \varphi_{\alpha k}^{K S}(\boldsymbol{r})=\epsilon_{\alpha \boldsymbol{k}}^{K S} \varphi_{\alpha \boldsymbol{k}}^{K S}(\boldsymbol{r})
$$

Devido a que o hamiltoniano é local, no sistema fictício de KS todos os elétrons estão sujeitos ao mesmo potencial, o que facilita a solução das equações de KS para um sólido cristalino.

\section{O Método de Ondas Planas}

Considerando os vetores de rede recíproca $\boldsymbol{G}_{n}$, que obedecem à relação $e^{i \boldsymbol{G}_{n} \cdot \boldsymbol{R}_{n}}=1$, no método de ondas planas o potencial efetivo é expandido em uma série de Fourier no espaço recíproco:

$$
V_{e f f}(\boldsymbol{r})=\sum_{\boldsymbol{G}_{n}} V_{e f f}^{\boldsymbol{G}_{n}} e^{i \boldsymbol{G}_{n} \cdot \boldsymbol{r}}
$$

Expandem-se também os orbitais de KS em ondas planas

$$
\varphi_{\alpha \boldsymbol{k}}^{K S}(\boldsymbol{r})=\sum_{\boldsymbol{G}_{n}} C_{\boldsymbol{k}+\boldsymbol{G}_{n}}^{\alpha} e^{i\left(\boldsymbol{k}+\boldsymbol{G}_{n}\right) \cdot \boldsymbol{r}}
$$


Das equações (3.29) e (3.30) na expressão (3.28), é possível mostrar que os coeficientes $C_{\boldsymbol{k}+\boldsymbol{G}_{n}}$ e os autovalores de KS são encontrados resolvendo:

$$
\sum_{\boldsymbol{G}_{n}}\left[\frac{1}{2}\left(\boldsymbol{k}+\boldsymbol{G}_{n}\right)^{2} \delta_{\boldsymbol{G}_{n}, \boldsymbol{G}_{n}{ }_{n}}+V_{\boldsymbol{G}_{n}, \boldsymbol{G}_{n}{ }_{n}}\right] C_{\boldsymbol{k}+\boldsymbol{G}_{n}}^{\alpha}=\epsilon_{\alpha \boldsymbol{k}}^{K S} C_{\boldsymbol{k}+\boldsymbol{G}_{n}}^{\alpha}
$$

onde,

$$
V_{\boldsymbol{G}_{n}, \boldsymbol{G}^{\prime}{ }_{n}} \int_{\text {cell.unit }} d^{3} r V_{\text {eff }}(\boldsymbol{r}) e^{i\left(\boldsymbol{G}_{n}, \boldsymbol{G}^{\prime}{ }_{n}\right) \cdot \boldsymbol{r}}
$$

A densidade do estado fundamental é determinada via o cálculo dos coeficientes $C_{\boldsymbol{k}+\boldsymbol{G}_{n}}^{\alpha}$ e as autoenergias $\in_{\alpha \boldsymbol{k}}^{K S}$.

\section{O Método das Bases Localizadas}

Este método baseia-se na suposição de que as funções de onda são periódicas em $\boldsymbol{k}$. Expandindo a função de onda de Kohn-Sham em bases localizadas:

$$
\varphi_{\alpha \boldsymbol{k}}^{K S}(\boldsymbol{r})=\sum_{\boldsymbol{R}_{n}} w^{\alpha k}\left(\boldsymbol{r}-\boldsymbol{R}_{n}\right) e^{i \boldsymbol{R}_{n} \cdot \boldsymbol{k}}
$$

sendo $\boldsymbol{R}_{n}$ o vetor de translação. Esta expansão considera funções $w^{\alpha k}\left(\boldsymbol{r}-\boldsymbol{R}_{n}\right)$ centradas em cada célula unitária indexada por $\boldsymbol{R}_{n}$.

Usando um conjunto de bases localizadas $\phi_{\mu}\left(\boldsymbol{r}-\boldsymbol{P}_{\mu}\right)$, centrada em átomos localizados em $\boldsymbol{P}_{\mu}$ expande-se $w^{\alpha k}\left(\boldsymbol{r}-\boldsymbol{R}_{n}\right)$ :

$$
w^{\alpha k}\left(\boldsymbol{r}-\boldsymbol{R}_{n}\right)=\sum_{\mu} C_{\mu}^{\alpha k} \phi_{\mu}\left(\boldsymbol{r}-\boldsymbol{P}_{\mu}-\boldsymbol{R}_{n}\right)
$$

Substituindo as expansões (3.33) e (3.34) na equação de KS, obtém-se:

$$
\sum_{\mu}\left[\sum_{\boldsymbol{R}_{n}} e^{i \boldsymbol{R}_{n} \cdot \boldsymbol{k}}\left(-\frac{1}{2} \nabla^{2}+V_{e f f}(\boldsymbol{r})\right) \phi_{\mu}\left(\boldsymbol{r}-\boldsymbol{P}_{\mu}-\boldsymbol{R}_{n}\right)\right] C_{\mu}^{\alpha k}
$$




$$
=\epsilon_{\alpha \boldsymbol{k}}^{K S} \sum_{\mu} \sum_{\boldsymbol{R}_{n}}\left[e^{i \boldsymbol{R}_{n} \cdot \boldsymbol{k}} \phi_{\mu}\left(\boldsymbol{r}-\boldsymbol{P}_{\mu}-\boldsymbol{R}_{n}\right)\right]
$$

Multiplicando a equação anterior por uma função base $\phi_{\nu}^{*}\left(\boldsymbol{r}-\boldsymbol{P}_{\nu}\right)$ e integrando no espaço real, a equação de KS é reduzida a:

$$
\sum_{\mu} H_{\nu \mu}(\boldsymbol{k}) C_{\mu}^{\alpha k}=\in_{\alpha \boldsymbol{k}}^{K S} \sum_{\mu} S_{\nu \mu}(\boldsymbol{k}) C_{\mu}^{\alpha k}
$$

onde,

$$
H_{\nu \mu}(\boldsymbol{k})=\sum_{\boldsymbol{R}_{\boldsymbol{n}}} e^{i \boldsymbol{R}_{n} \cdot \boldsymbol{k}}\left[\int d^{3} r \phi_{\nu}^{*}\left(\boldsymbol{r}-\boldsymbol{P}_{\nu}\right)\left(-\frac{1}{2} \nabla^{2}+V_{e f f}(\boldsymbol{r})\right) \phi_{\mu}\left(\boldsymbol{r}-\boldsymbol{P}_{\mu}-\boldsymbol{R}_{n}\right)\right]
$$

e,

$$
S \nu \mu(\boldsymbol{k})=\sum_{\boldsymbol{R}_{n}} e^{i \boldsymbol{R}_{n} \cdot \boldsymbol{k}}\left[\int d^{3} r \phi_{\nu}^{*}\left(\boldsymbol{r}-\boldsymbol{P}_{\nu}\right) \phi_{\mu}\left(\boldsymbol{r}-\boldsymbol{P}_{\mu}-\boldsymbol{R}_{n}\right)\right]
$$

Normalmente, as funções base $\phi_{\nu}\left(\boldsymbol{r}-\boldsymbol{P}_{\nu}\right)$ possuem a forma de orbitais atômicos, de forma que estas funções constituem uma base de orbitais atômicos localizados (LCAO).

O método de ondas planas, de forma geral, é mais robusto já que as ondas planas carregam a informação de periodicidade do sistema em relação ao método de bases localizadas que além de ser mais leve computacionalmente, possui uma flexibilidade na escolha das bases permitindo a simulação de sistemas maiores com um menor custo computacional.

\subsection{Pseudopotencial de Norma Conservada}

O método dos pseudopotenciais permite descrever o efeito dos átomos que não participam das ligações químicas, os quais denominam-se elétrons de caroço, como um potencial efetivo de uma partícula que atua sob os elétrons de valência. Desta forma evita-se desenvolver cálculos mais completos os quais incluem todos 
os elétrons na descrição de um material. Geralmente, este cálculos exigem o uso de uma malha muito fina que defina o espaço e permita descrever as fortes variações das funções de onda nas proximidades dos núcleos e portanto um alto custo computacional. Entretanto, os pseudopotenciais quando usados no formalismo da DFT com orbitais atômicos localizados fornecem simulações com alta precisão com custo computacional relativamente baixo.

Os pseudopotenciais foram obtidos pela primeira vez por Philips e Kleimann[15] a partir de uma metodologia baseada no método OPW[16] para cálculos de banda de energia. Esta metodologia foi a primeira em gerar um pseudopotencial através de um processo $a b$ - initio do qual foram obtidos vários pseudopotenciais empregados em cálculos de estrutura eletrônica de moléculas dentro da aproximação Hattre-Fock[17,18,19,20]. Basicamente o trabalho de Philips e Kleimann[15] baseia-se na combinação de pseudo-funções de onda de valência $\left|\phi_{v}\right\rangle$ com funções de onda de caroço $\left|\psi_{c}\right\rangle$ :

$$
\left|\psi_{n}\right\rangle=\left|\phi_{v}\right\rangle+\sum_{c} a_{c}\left|\psi_{c}\right\rangle
$$

sujeita à condição que cada função de base $\left|\psi_{n}\right\rangle$ seja ortogonal ao estado de caroço $\left|\psi_{c}\right\rangle$, ou seja, $\left\langle\psi_{c} \mid \psi_{n}\right\rangle=0$

A partir da proposição inicial de Philips e Kleinman[15] vários autores fizeram modificações[21] que culminaram com as propostas "norm conserving" ou pseudopotenciais de norma conservada. Estes fazem parte dos enfoques mais utilizados nos cálculos com a DFT, como por exemplo, as formulações de Bachelet, Hamann e Schluter (BHS), e de Troullier-Martins (T-M). A construção desses pseudopotenciais foi proposta por Zunger e Cohen e consiste em usar a equação inversa tipo Schrödinger para o problema de íon livre $(V(r)=0)$ com a inclusão de pseudopotencial. Considerando a parte radial da equação do íon livre (unidades 
atômicas):

$$
\left\{-\frac{1}{2} \frac{d^{2}}{d r^{2}}+\frac{l(l+1)}{2 r^{2}}+V_{p s}^{l}(r)\right\} P_{p s, l}(r)=\varepsilon_{l} P_{p s, l}(r)
$$

Invertendo tem-se que:

$$
V_{p s}^{l}(r)=\varepsilon_{l}-\frac{l(l+1)}{2 r^{2}}+\frac{1}{2} \frac{P_{p s, l}^{\prime \prime}(r)}{P_{p s, l}(r)},
$$

onde $V_{p s}^{l}$ é a componente do pseudopotencial blindada com um dado valor $l, \varepsilon_{l}$ é o autovalor da energia de valência como o número quântico orbital $l, P_{p s, l}$ é a pseudofunção radial e $P_{p s, l}^{\prime \prime}$ é sua segunda derivada.

A formulação geral consiste em achar a pseudofunção apropriada tal que seja idêntica à função efetiva para $r$ maior que uma distância determinada do núcleo denominada raio de corte $r_{c}$,

$$
P_{p s, l}=\left\{\begin{array}{cc}
r^{l+t} f(r), & r<r_{c} \\
P_{l}(r), & r>r_{c},
\end{array}\right.
$$

onde $P_{l}(r)=r R_{l}(r) \operatorname{com} R_{l}(r)$ a parte radial da função de onda obtida com todos os elétrons. A função $R_{l}(r)$ é chamada de função all alectron, e pode ser obtida via solução das equações de Kohn-Sham usando um potencial de exchange-correlação. A função $f(r)$ é escolhida com a forma:

$$
f(r)=e^{\sum_{i=0}^{n} a_{i} r^{i}}
$$

O parâmetro $t$ pode ter valores $t=1$ ou $t=2$. No caso $t=2$, o potencial terá um comportamento assintótico do tipo $B e / r^{2}$, com $B e>0$, quando $r \rightarrow 0$ e no caso $t=1$ o comportamento será do tipo $1 / r$. Agora, colocando $\alpha_{1}=0$, é possível obter potenciais sem singularidades. O coeficiente $\alpha_{i}$ é determinado a partir da coincidência da verdadeira função e da pseudofunção no ponto $r_{c}$ podendo incluir a exigência da coincidência da primeira e da segunda derivadas. Pela outra condição, 
a conservação da norma, a pseudofunção integrada de zero à $r=r_{c}$ deve ser igual ao valor desta integral para a densidade de carga verdadeira.

Em síntese, os pseudopotenciais de norma conservada apresentam as seguintes propriedades:

i. Os autovalores $\varepsilon_{i}$ obtidos para os estados de valência atômicos devem ser por construção idênticos aos autovalores $\varepsilon_{i}^{p s}$ obtidos com o pseudopotencial;

ii. as autofunções relativas à solução exata e à solução obtida com o pseudopotencial devem ser iguais para $r>r_{c}$;

iii. as integrais de 0 a $r, r>r_{c}$ das densidades de carga da solução exata devem ser iguais às das soluções obtidas com o pseudopotencial;

iv. a derivada logarítmica da pseudofunção deve convergir para a da função de onda exata para $r>r_{c}$.

Deve-se observar que quando se fala de função de onda exata se faz referência àquela obtida com cálculos incluindo todos os elétrons, inclusive do caroço. A propriedade (iii) garante, através do teorema de Gauss, que o potencial eletrostático produzido em $r>r_{c}$, é o mesmo produzido pela densidade exata ou pela pseudodensidade. A propriedade (iv) assegura que medidas de espalhamento são reproduzidas com erro mínimo. Um ponto importante é que as condições (iii) e (iv) garantem, de certa forma, a capacidade destes potenciais serem transferíveis, ou seja, são as condições de transferibilidade. Estas podem ser expressadas por uma única identidade:

$$
-2 \pi\left[(r R)^{2} \frac{d}{d \varepsilon} \frac{d}{d r} \ln R\right]_{r c}=4 \pi \int_{0}^{r_{c}}|R|^{2} r^{2} d r
$$

onde $R$ é a solução da equação radial de Schrödinger para energia E. Como exigimos que as funções de onda sejam normalizadas, dentro de uma esfera de raio $r>r_{c}$, 
o lado direito da equação acima é igual tanto para a pseudofunção quanto para a função exata. 
$\Gamma_{\text {Capítulo }}$

\section{Implementação do Acoplamento}

\section{Spin-Órbita no Código SIESTA}

O desenvolvimento teórico que fez das simulações computacionais uma poderosa ferramenta para o estudo e predição do comportamento de sistemas físicos, no caso particular do estudo das propriedades eletrônicas de sistemas cristalinos, associa-se à introdução do formalismo dos pseudopotenciais nos métodos geralmente usados. Os pseudopotenciais permitem modelar a matéria como um sistema formado pelos elétrons de valência sob a ação de um potencial efetivo que leva em consideração a contribuição dos elétrons de caroço, desta forma, elimina-se a necessidade de fazer uma descrição all electron que leve em consideração a contribuição tanto dos orbitais de valência quanto os de caroço de forma explícita. Consequentemente, os estudos all electron restringem-se a sistemas de poucos átomos, enquanto que os métodos como o Hartree-Fock ou a DFT usados junto com os pseudopotenciais permitem estudar estruturas compostas por muitos átomos.

O formalismo dos pseudopotenciais de norma conservada proposto por Zunger e Cohen em (1978)[22] usado dentro da DFT, permite obter uma descrição apropriada das propriedades eletrônicas dos sólidos cristalinos e moléculas compostos 
por muitos átomos com um custo computacional relativamente baixo. Contudo, o enfoque original foi desenvolvido a partir dos princípios da mecânica quântica não relativística usando a equação inversa do tipo Schrödinger. Portanto, este formalismo não permite descrever efeitos presentes em sistemas de átomos pesados tal como o SOC.

Por outra parte, Kleinman[23] desenvolveu uma generalização para a obtenção dos pseudopotenciais de norma conservada no contexto da teoria relativística da mecânica quântica. Este formalismo parte da equação de Dirac para um potencial central permitindo a inclusão de interações relativísticas tais como a correção da massa relativística e o SOC.

Com o formalismo da equação de Dirac explicado no capítulo 1, o entendimento do problema eletrônico nos sólidos cristalinos e as ferramentas para resolvê-lo apresentadas no capítulo 2, tem-se a estrutura teórica necessária para incluir o formalismo proposto por Kleinman dentro da teoria do funcional da densidade. Nesta ordem, apresenta-se a forma na qual, a partir da equação radial de Dirac, obtém-se o pseudopotencial relativístico de norma conservada.

Neste trabalho, foi desenvolvida a implementação do SOC no código SIESTA a partir do método proposto por Férnandez-Seivane et al.[24], para a inclusão do pseudopotencial relativístico na equação de Kohn-Sham com LCAO.

Neste capítulo, serão apresentados os aspectos matemáticos do método usado, generalidades do código SIESTA e os rigorosos testes que verificam o bom funcionamento da implementação. 


\subsection{Formalismo do Pseudopotencial Relativístico de Norma Conservada}

O formalismo do pseudopotencial de norma conservada apresentado no capítulo 3 permite obter uma descrição adequada das propriedades eletrônicas dos sólidos cristalinos levando em conta a contribuição do caroço como um potencial efetivo que atua sob os elétrons de valência. Contudo, para a descrição dos efeitos relativísticos de primeira ordem precisa-se de um pseudopotencial de norma conservada dentro da aproximação (2.20). Para construir este pseudopotencial, denominado pseudopotencial relativístico de norma conservada, pode-se fazer uma descrição para os elétrons de valência a partir da equação radial de Dirac a qual foi escrita como um sistema de equações (2.53) e (2.54).

Ao considerar $\epsilon$ como a energia dos elétrons de valência e $V(\boldsymbol{r})$ como o potencial efetivo sentido pelos elétrons fora da região de caroço, obtém-se uma relação entre as soluções $G(\boldsymbol{r})$ e $F(\boldsymbol{r})$ da equação de Dirac:

$$
F(\boldsymbol{r})=\frac{1}{2} \lambda\left[\frac{d G(\boldsymbol{r})}{d r}+(\kappa / r) G(\boldsymbol{r})\right]
$$

Deste modo, obtém-se uma equação para a componente $G(\boldsymbol{r})$ que associa-se com a função de onda de energia negativa $g(\boldsymbol{r})=r^{-1} G(\boldsymbol{r})$ da equação de Dirac:

$$
\frac{1}{2}\left(\frac{d^{2} G}{d r^{2}}+\frac{\kappa(\kappa+1)}{r^{2}} G\right)+[V(r)-\epsilon] G=0
$$

Para átomos pesados a componente radial da função de onda de energia negativa $f(\boldsymbol{r})=r^{-1} F(\boldsymbol{r})$ mistura-se fortemente com a componente radial da função de onda de energia positiva. A equação (4.2) corresponde a um análogo relativístico da equação radial de Kohn-Sham. No entanto, de acordo com a tabela 2.1 o operador 
SOC possui dois autovalores:

$$
\kappa=\left\{\begin{array}{cc}
j+\frac{1}{2}=l, & \kappa>0 \\
-\left(j+\frac{1}{2}\right)=-(l+1), & \kappa<0
\end{array}\right.
$$

de forma que, para cada valor de $\kappa$ existe uma função de onda radial que se denota em termos do valor do momento angular como: $G_{l+\frac{1}{2}}(\boldsymbol{r})$ e $G_{l-\frac{1}{2}}(\boldsymbol{r})$.

De forma análoga aos desenvolvimentos de T-M e BHS para a obtenção do pseudopotencial não relativístico pode-se construir uma generalização para o caso relativístico que satisfaz as condições de um pseudopotenical de norma conservada. Será ilustrado este procedimento a partir do esquema de Bachelet-Hamann-Schluter (HBS)[25]. O pseudopotencial de norma conservada proposto por HSB foi obtido a partir de cálculos all electron que se iniciam com a solução da equação de KohnSham. Portanto, a descrição relativística inicia com a solução all electron da equação de Dirac (4.2), onde a função de onda e energia obtidas deste cálculo denominam-se exatas. O potencial $V(\boldsymbol{r})$ modifica-se de acordo com as condições que têm que ser satisfeitas por um pseudopotencial de norma conservada. Consequentemente, cortase a singularidade do potencial em $r=0$ com uma função analítica de corte dada pela expressão:

$$
f\left(r / r_{c_{l}}\right)=\exp \left(-\frac{r}{r_{c_{l}}}\right)^{\lambda},
$$

onde $r_{c_{l}}$ é o raio de corte tipicamente escolhido de 0,5 a 1,0 vezes o raio do pico mais externo da função de onda exata[25]. Os pseudopotenciais usados comumente no código SIESTA são não relativísticos. Porém, as correções do SOC implicam que não necessariamente os $r_{c_{l}}$ usados para a construção destes são os adequados para gerar o pseudopotencial que leva em consideração o SOC, principalmente quando os átomos são pesados. Dado que $r_{c_{l}}$ define a região de caroço e a qualidade do pseudopotencial, os cálculos de estrutura eletrônica feitos neste trabalho exigiram a mudança e posterior otimização dos raios de corte para a construção dos pseudopotenciais que 
levassem em conta o SOC para cada espécie atômica usada.

O valor da constante $\lambda$ foi obtido pela primeira vez por Hamann, Schluter e Chiang (HSC)[26] e a partir da otimização dos resultados para diversos átomos HSC obtiveram um valor igual a 4. No entanto, HSB usando métodos empíricos em uma grande variedade de átomos corrigiram o valor para 3,5[27].

Note-se que $\lim _{r \rightarrow \infty} f\left(r / r_{c_{l}}\right)=0$ e $\lim _{r \rightarrow 0} f\left(r / r_{c_{l}}\right)=1$, e caem rapidamente a partir de $r \cong r_{c_{l}}$. Desta forma o potencial original $V(\boldsymbol{r})$ reescreve-se como:

$$
V_{p s}^{1\left(l \pm \frac{1}{2}\right)}(\boldsymbol{r})=\left[1-f\left(r / r_{c}\right)\right] V(\boldsymbol{r})+C_{l \pm \frac{1}{2}} f\left(r / r_{c}\right)
$$

A constante $C_{l \pm \frac{1}{2}}$ é ajustada para obter a menor solução sem nós da equação radial de Dirac contendo o pseudopotencial modificado $V_{p s}^{1\left(l \pm \frac{1}{2}\right)}(\boldsymbol{r})$ :

$$
\left[\frac{1}{2} \frac{d^{2}}{d r^{2}}+V_{p s}^{1\left(l \pm \frac{1}{2}\right)}(\boldsymbol{r})+\frac{\kappa(\kappa+1)}{2 r^{2}}\right] G_{p s}^{1\left(l \pm \frac{1}{2}\right)}(\boldsymbol{r})=\epsilon_{1\left(l \pm \frac{1}{2}\right)} G_{p s}^{1\left(l \pm \frac{1}{2}\right)}(\boldsymbol{r})
$$

de forma que a energia $\epsilon_{1\left(l \pm \frac{1}{2}\right)}$ é igual à energia exata e a propriedade $(i)$ dos pseudopotenciais de norma conservada; descrita na seção 3.4, fica satisfeita. Consequentemente, $G_{p s}^{1\left(l \pm \frac{1}{2}\right)}(\boldsymbol{r})$ satisfaz a mesma equação e as mesmas condições de contorno que $G_{l \pm \frac{1}{2}}(\boldsymbol{r})$, portanto para $r>r_{c}$ a pseudofunção e a função exata só diferem de uma constante multiplicativa:

$$
\gamma_{1\left(l \pm \frac{1}{2}\right)} G_{p s}^{1\left(l \pm \frac{1}{2}\right)} \rightarrow G_{l \pm \frac{1}{2}}(\boldsymbol{r})
$$

No entanto, a pseudofunção não satisfaz a propriedade $(i i)$. Portanto, modifica-se a pseudofunção através da expressão:

$$
G_{p s}^{(2)\left(l \pm \frac{1}{2}\right)}(r)=\gamma_{\left(l \pm \frac{1}{2}\right)}\left[G_{p s}^{1\left(l \pm \frac{1}{2}\right)}(r)+\delta g_{l \pm \frac{1}{2}}\left(r / r_{c}\right)\right]
$$

onde a constante garante as condições de normalização e a função $g_{l \pm \frac{1}{2}}\left(\frac{r}{r_{c}}\right)$ é definida 
como:

$$
g_{l \pm \frac{1}{2}}\left(\frac{r}{r_{c}}\right)=\left(\frac{r}{r_{c}}\right)^{\left(l \pm \frac{1}{2}\right)+1} \exp \left(-\left(\frac{r}{r_{c}}\right)^{l \pm \frac{1}{2}}\right) .
$$

Finalmente o pseudopotencial para cada valor do momento angular total é obtido pela inversão da equação radial de Dirac:

$$
V_{p s}^{(2)\left(l \pm \frac{1}{2}\right)}(r)=\epsilon_{1\left(l \pm \frac{1}{2}\right)}-\frac{\kappa(\kappa+1)}{2 r^{2}}+\frac{1}{2} \frac{G_{p s}^{\prime \prime(2)\left(l \pm \frac{1}{2}\right)}(r)}{G_{p s}^{(2)\left(l \pm \frac{1}{2}\right)}(r)}
$$

Para obter o pseudopotencial "desblindado", os potenciais de Coulomb e de troca e correlação são subtraídos do potencial $V_{p s}^{2 l}(r)$. Portanto, o pseudopotencial que além de levar em conta os efeitos relativísticos de primeira ordem, substitui o caroço do átomo para cada valor do momento angular total é dado por:

$$
V_{p s}^{i o n\left(l \pm \frac{1}{2}\right)}(r)=V_{p s}^{(2)\left(l \pm \frac{1}{2}\right)}(r)-\frac{4 \pi}{r} \int_{0}^{r} \rho\left(r^{\prime}\right) r^{\prime 2} d r^{\prime}-4 \pi \int_{r}^{\infty} \rho\left(r^{\prime}\right) r^{\prime} d r^{\prime}-\frac{\delta E_{x c}[\rho]}{\delta \rho(r)}
$$

Assim, mostra-se que a partir da solução auto-consistente da equação de Dirac all electron para um átomo, o procedimento para gerar o pseudopotencial não relativístico pode ser estendido para levar em conta os efeitos relativísticos de primeira ordem, permitindo desta forma o estudo da estrutura fina através de cálculos de estrutura eletrônica. No entanto, a forma como este pseudopotencial é incluído no formalismo LCAO não é evidente e, portanto, é necessária sua especificação. Usando operadores de projeção em termos da função de onda angular (2.39) o pseudopotencial relativístico pode ser escrito como:

$$
\hat{V}_{p s}^{i o n}(r)=\sum_{l, m}\left[\left|\chi_{M}^{J}\right\rangle V_{p s}^{i o n\left(l+\frac{1}{2}\right)}(r)\left\langle\chi_{M}^{J}|+| \chi_{M^{\prime}}^{J^{\prime}}\right\rangle V_{p s}^{i o n\left(l-\frac{1}{2}\right)}(r)\left\langle\chi_{M^{\prime}}^{J^{\prime}}\right|\right]
$$

onde $J=l+\frac{1}{2}, M=m+\frac{1}{2}, J^{\prime}=l-\frac{1}{2}$ e $M^{\prime}=m-\frac{1}{2}$. Substituindo as funções de 
onda angulares reescreve-se o pseudopotencial:

$$
\hat{V}_{p s}^{i o n}=\hat{V}_{s c}+\hat{V}_{S O}=\sum_{l, m}\left[V_{s c}^{l}(\boldsymbol{r}) \boldsymbol{I}_{\sigma}+V_{S O}^{l}(\boldsymbol{r}) \boldsymbol{L} \cdot \boldsymbol{S}\right]|l, m\rangle\langle l, m|,
$$

sendo $\boldsymbol{I}_{\sigma}$ o operador identidade no espaço de spin,

$$
V_{S O}^{l}(\boldsymbol{r})=\frac{2}{2 l+1}\left[V_{p s}^{(2)\left(l+\frac{1}{2}\right)}(r)-V_{p s}^{(2)\left(l-\frac{1}{2}\right)}(r)\right]
$$

e

$$
V_{s c}^{l}(\boldsymbol{r})=\frac{1}{2 l+1}\left[(l+1) V_{p s}^{(2)\left(l+\frac{1}{2}\right)}(r)+l V_{p s}^{(2)\left(l-\frac{1}{2}\right)}(r)\right] .
$$

Note-se que a parte escalar do pseudopotencial $\hat{V}^{s c}$, além de conter o convencional pseudopotencial não relativístico leva em consideração as correções escalares relativísticas de primeira ordem. Finalmente, o hamiltoniano eletrônico pode ser expresso como:

$$
\hat{\boldsymbol{H}}=\hat{\boldsymbol{T}}+\hat{\boldsymbol{V}}^{s c}+\hat{\boldsymbol{V}}^{S O}+\hat{\boldsymbol{V}}^{H}+\hat{\boldsymbol{V}}^{x c}
$$

onde $\hat{T}$ representa a energia cinética, $\hat{V}_{s c}$ o escalar relativístico, $\hat{V}_{S O}$ o termo de interação spin-órbita, $\hat{V}_{H}$ o termo de Hartree e $\hat{V}_{e x}$ o potencial de troca e correlação. Portanto, este hamiltoniano é uma matriz 2X2 no espaço spin, dado por:

$$
\hat{H}=\left(\begin{array}{cc}
\hat{H}^{\uparrow \uparrow} & \hat{H}^{\uparrow \downarrow} \\
\hat{H}^{\downarrow \uparrow} & \hat{H}^{\downarrow \downarrow}
\end{array}\right)
$$

Os blocos não diagonais surgem do potencial de troca e correlação (quando o sistema considerado tem spin não colinear) e do potencial spin-órbita.

\subsubsection{LCAO e aproximação "on-site" da interação spin-órbita}

O formalismo desenvolvido no capitulo anterior para o uso de orbitais atômicos localizados pode ser generalizado com a finalidade de considerar o spin. 
Neste caso, a expansão da função de onda em termos de orbitais atômicos localizados pode ser escrita como:

$$
\left|\psi_{n}\right\rangle=\sum_{i}\left(\begin{array}{c}
c_{n, i}^{\uparrow} \\
c_{n, i}^{\downarrow}
\end{array}\right)\left|\phi_{i}\right\rangle
$$

Portanto, a equação de KS toma a forma:

$$
\left[\begin{array}{cc}
H_{i j}^{\uparrow \uparrow}-E_{n} S_{i j} & H_{i j}^{\uparrow \downarrow} \\
\hat{H}^{\downarrow \uparrow} & H_{i j}^{\downarrow \downarrow}-E_{n} S_{i j}
\end{array}\right]\left[\begin{array}{c}
c_{n, i}^{\uparrow} \\
c_{n, i}^{\downarrow}
\end{array}\right]=0 .
$$

Finalmente, o termo do pseudopotencial associado à contribuição spin-órbita é dado por:

$$
V_{i j}^{S O}=\left\langle\phi_{i}\left|\hat{\boldsymbol{V}}^{S O}\right| \phi_{j}\right\rangle=\sum_{k, l_{k}, M_{k}}\left\langle\phi_{i}\left|\hat{\boldsymbol{V}}_{l_{k}}^{S O} L \cdot S\right| l_{k}, M_{k}\right\rangle\left\langle l_{k}, M_{k} \mid \phi_{j}\right\rangle
$$

ou:

$$
V_{i j}^{S O}=\frac{1}{2} \sum_{k, l_{k}>0, M_{k}}\left\langle R_{n_{i}, l_{i}}\left|\hat{\boldsymbol{V}}_{l_{k}}^{S O}\right| R_{n_{j}, l_{j}}\right\rangle\left\langle l_{i}, M_{i}|L \cdot S| l_{k}, M_{k}\right\rangle\left\langle l_{k}, M_{k} \mid l_{j}, M_{j}\right\rangle
$$

Note-se que a implementação do pseudopotencial SOC da forma (4.21) exige a resolução de integrais de três centros. Consequentemente, Férnandez-Seivane et al.[24], propuseram a denominada aproximação "on-site", na qual são considerados unicamente termos centrados no mesmo sítio, nesta visão obtem-se:

$$
V_{i j}^{S O}=\frac{1}{2}\left\langle R_{n_{i}, l_{i}}\left|\hat{\boldsymbol{V}}_{l_{i}}^{S O}\right| R_{n_{j}, l_{j}}\right\rangle\left\langle l_{i}, M_{i}|L \cdot S| l_{j}, M_{j}\right\rangle \delta_{l_{i}, l_{j}}
$$

Desta forma foi implementado o SOC no código computacional SIESTA. Será mostrado que embora exista um termo de SOC associado como o "hopping" dos segundos vizinhos, o código no formato implementado fornece resultados que concordam tanto com outros códigos quanto com dados experimentais. É importante mencionar que no código SIESTA usam-se os harmônicos esféricos reais. Entretanto, 
neste trabalho foram usadas transformações unitárias diferentes às propostas por Férnandez-Seivane et al.[24].

Algumas simetrias úteis dos elementos de matriz do hamiltoniano e de sua parte SO podem ser usadas. Como o hamiltoniano é hermitiano, seus elementos de matriz satisfazem a relação:

$$
H_{i j}^{\sigma \sigma^{\prime}}=\left(H_{i j}^{\sigma^{\prime} \sigma}\right)^{*}
$$

Entretanto, a contribuição do SOC é anti-hermitiana na caixa de spin, ou seja:

$$
H_{i j}^{\sigma \sigma^{\prime}}=-\left(H_{i j}^{\sigma^{\prime} \sigma}\right)^{*}
$$

Esta propriedade tem consequências importantes para o cálculo da energia total.

\subsubsection{Matriz de Densidade e Energia Total}

A densidade de carga também pode ser escrita em termos da base LCAO como:

$$
n(\boldsymbol{r})=\sum_{n} f n \psi_{n}(\vec{r}) \psi_{n}(\boldsymbol{r})^{\dagger}=\sum_{i j} \phi_{i}\left(\boldsymbol{r}-\boldsymbol{d}_{i}\right) \phi_{j}^{*}\left(\boldsymbol{r}-\boldsymbol{d}_{j}\right) p_{i j}
$$

onde $f_{n}$ representa a ocupação do estado de Kohn-Sham $\psi_{n}(\boldsymbol{r})$ e $\rho_{i j}$ é uma matriz $2 \times 2$ que contêm os produtos dos coeficientes da função de onda:

$$
\rho_{i j}^{\sigma \sigma^{\prime}}=\sum_{n} f_{n} c_{n, i}^{\sigma} c_{n, j}^{\sigma^{\prime} *}
$$

A contribuição eletrônica à energia total pode ser expressa como a somatória das contribuições na estrutura de bandas dos elementos matriciais na caixa de spin do hamiltoniano eletrônico. Esta contribuição escrita em termos da base LCAO toma 
a forma:

$$
E_{e}=\sum_{n} f_{n}\left\langle\psi_{n}|\hat{H}| \psi_{n}\right\rangle=\sum_{i, j, \sigma, \sigma^{\prime}} H_{i j}^{\sigma \sigma^{\prime}} \rho_{i j}^{\sigma^{\prime} \sigma}
$$

que também pode ser expressa como:

$$
\sum_{i j}\left\{H_{i j}^{\uparrow \uparrow} \rho_{j i}^{\uparrow \uparrow}+H_{i j}^{\downarrow \downarrow} \rho_{j i}^{\downarrow \downarrow}+2 R e\left[\left(V_{i j}^{x c \uparrow \downarrow}-V_{i j}^{S O \uparrow \downarrow}\right)\left(\rho_{j i}^{\uparrow \downarrow}\right)^{*}\right]\right\}
$$

onde isolamos a contribuição não diagonal no espaço de spin. Isto surge só do SOC e do potencial de troca e correlação, sempre que as contribuições não colineares do spin estão presentes. A contribuição do SOC à energia total é dado por:

$$
E^{S O}=\operatorname{Tr} \sum_{i, j} V_{i j}^{S O} \rho_{i j}
$$

já que não há termos de dupla contagem.

\subsubsection{Forças}

Uma importante consequência da aproximação on-site é que o SOC não da origem a uma contribuição explícita às forças, embora esteja sempre presente uma contribuição implícita devido à modificação da função de onda auto-consistente. De acordo com o teorema de Hellmann-Feynman a contribuição do SOC às forças é obtida simplesmente diferenciando a energia em relação às coordenadas atômicas:

$$
-\boldsymbol{F}_{k}^{S O}=\nabla_{k} E^{S O}=T_{r} \sum_{i, j}\left\{\left[\nabla_{k} \hat{V}_{i j}^{S O}\right] \rho_{j i}+V_{i j}^{S O}\left[\boldsymbol{\nabla}_{k} \hat{\rho}_{j i}\right]\right\}
$$

onde ambos orbitais $i$ e $j$ estão centrados na mesma posição atômica $\boldsymbol{d}_{k}$ e $\boldsymbol{\nabla}_{k}=\boldsymbol{\nabla}_{\boldsymbol{d}_{k}}$. Contudo, ambas contribuições às forças do SOC na anterior equação desaparecem. A primeira integral é idêntica a zero enquanto as integrais centrais não dependem 
da posição atômica:

$$
\nabla_{k}\left\langle R_{n i, l i}\left|\bar{V}_{l k}^{S O}\right| R_{n j, l j}\right\rangle \equiv 0
$$

O segundo termo pode ser reescrito como:

$$
-\sum_{i j}\left(\varepsilon_{i j}^{S O, \uparrow \uparrow}+\varepsilon_{i j}^{S O, \downarrow \downarrow}\right) \nabla_{k} S_{j i}
$$

onde $\varepsilon_{i j}^{S O, \sigma \sigma^{\prime}}$ são componentes da contribuição do SOC à matriz densidade de energia:

$$
\varepsilon_{i j}^{S O}=\frac{1}{2} \sum_{l, m}\left(S_{i l}^{-1} V_{l m}^{S O} \rho_{m j}+\rho_{i l} V_{l m}^{S O} S_{m j}^{-1}\right) .
$$

Entretanto, $\varepsilon^{S O}$ é assimétrico com respeito aos índices dos orbitais em contraste com a matriz de sobreposição que é simétrica. O segundo termo também desaparece. Em uma forma similar podemos demonstrar que o SOC na aproximação on-site não introduz nenhuma contribuição ao estresse.

\subsection{Código Siesta}

Soler et al.[28] desenvolveram o código SIESTA (Spanish Initiative for Electronic Simulations with Thousands of Atoms) como proposta de implementação de um método capaz de realizar cálculos eficientes de estrutura eletrônica ab-initio e simulações de dinâmica molecular em moléculas e sólidos. Este método está baseado na introdução do formalismo dos pseudopotenciais de norma conservada na DFT. A eficiência do código SIESTA decorre do uso de um conjunto de bases rigorosamente localizadas (que denominamos LCAO no capitulo 2) e da aplicação de escalonamento linear no algoritmo. Uma característica relevante do código associa-se com a possibilidade de sintonizar a precisão e o custo computacional para desenvolver cálculos exploratórios rápidos para simulações altamente precisas com a mesma qualidade de outras abordagens, como ondas planas e métodos all electron. 
A possibilidade de tratar grandes sistemas a partir de cálculos de primeiros princípios abriu novas oportunidades em muitas disciplinas. Contudo, a versão livre deste código não leva em conta a contribuição do SOC, portanto, embora permita estudar uma enorme quantidade de sistemas, não possibilita o estudo de sistemas de atual interesse na física da matéria condensada tal como as fases quânticas topológicas geradas pelo SOC.

O código SIESTA fornece a estrutura adequada para a implementação do formalismo proposto por Férnandez-Seivane et al.[24], que permite a inclusão dos efeitos do SOC nos cálculos de estrutura eletrônica. Tendo em vista que o SOC gera um hamiltoniano com elementos não diagonais (reais e imaginários) em relação à caixa de spin, precisa-se do uso do spin não colinear para realizar cálculos que considerem o SOC. Entretanto, nas versões distribuídas gratuitamente para os acadêmicos a opção de spin não colinear não funciona. Portanto, o primeiro passo no desenvolvimento do processo de implementação foi solucionar os erros no spin não colinear do código livre o que gerou dificuldades na realização deste trabalho. 


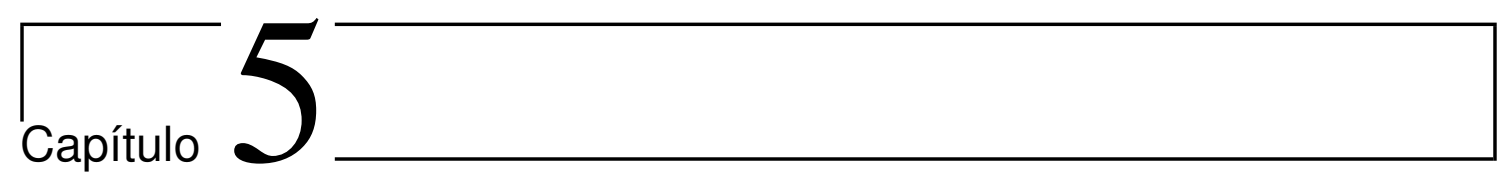

\section{Aplicações}

Neste trabalho, foram feitos diversos testes que verificaram a efetividade da implementação da aproximação "on-site" do SOC no código SIESTA. Demonstrou-se que esta aproximação dentro da teoria do funcional da densidade permite descrever tanto qualitativamente quanto quantitativamente os efeitos do SOC em sistemas cristalinos. O uso do formalismo dos pseudopotenciais e de bases LCAO permite estudar sistemas muito grandes com um baixo custo computacional.

Foram estudados efeitos gerados pelo SOC tais como o spin-splitting (Gap $\mathrm{SOC}$ ) em semicondutores e a formação de estados topológicos em $\mathrm{Bi}_{2} \mathrm{Se}_{3}$. Para isto, foram selecionados sistemas que podem-se considerar como representantes dos grupos de materiais conhecidos por apresentar fortes efeitos associados ao SOC. Os resultados obtidos concordam com resultados já publicados na literatura.

\subsection{Gap SOC}

Como foi discutido no capítulo 1 , os semicondutores com estrutura de diamante ou Zincblende, fornecem um exemplo claro da forma como o SOC afeta os níveis de energia dos estados de Bloch em um sólido cristalino. Estes sistemas 
em particular apresentam uma hexa-degenerescência no topo da banda de valência $\Gamma_{15}^{v}$ de caráter $p$ (momento angular orbital $l=1$ ). Quando se considera o SOC quebra-se a degenerescência de forma que esta banda é desdobrada. O levantamento da degenerescência no ponto $\Gamma$ do topo da banda de valência devido ao SOC leva à obtenção das bandas $\Gamma_{8}\left(\right.$ com momento angular total $\left.j=\frac{3}{2}\right)$ quatro vezes degenerada e $\Gamma_{7}$ (momento angular total $j=\frac{1}{2}$ ) bidegenerada, separadas pelo gap SOC, $\Delta_{0}$, como é ilustrado na figura 5.1. Esta é a característica principal dos efeitos associados ao SOC nos semicondutores dos grupos IV e III-V. Entretanto, existe uma diferença fundamental em relação ao número de espécies atômicas presentes na estrutura. Na estrutura constituída por dois elementos diferentes tal como o GaAs, quebra-se a simetria de inversão espacial de forma que a simetria cúbica da estrutura de diamante $O_{h}$ reduz-se à simetria $T_{d}$ que possui a metade de operações de simetria. Consequentemente, nos semicondutores com estrutura Zincblende, além de ser levantada a degenerescência de spin na banda $\Gamma_{15}^{v}$ presente nas estruturas compostas por um único elemento químico (também conhecida como estrutura de diamante), quebra-se a degenerescência nas direções $L \rightarrow \Gamma$ e $\Gamma \rightarrow X$ da banda $\Gamma_{15}^{v}$ como é esquematizado na figura 5.2. Contudo, existem outras evidências dos efeitos do SOC nos semicondutores com simetria zincblende, por exemplo, as quebras de degenerescência das bandas $\Gamma_{15}^{c}, L_{3}^{v}$ e $L_{3}^{c}$ representadas na estrutura de bandas do GaAs na figura 5.1c. Estas quebras de degenerescência caracterizam-se pelos gap SO $\bar{\Delta}_{0}, \Delta_{1}$ e $\bar{\Delta}_{1}$.

Foi usado o potencial de troca e correlação LDA, meshcutoff de 400 Ry, 50 pontos $k$ na grid. Os valores dos gap SO concordam com os dados experimentais[29] (ver tabela 5.1). O uso do potencial de troca-correlação LDA fornece valores de gap de energia da estrutura de bandas dos semicondutores inferiores aos experimentais, de modo que a subestimação do gap aumenta com a inclusão do SOC como evidenciado na figura 5.3a. 

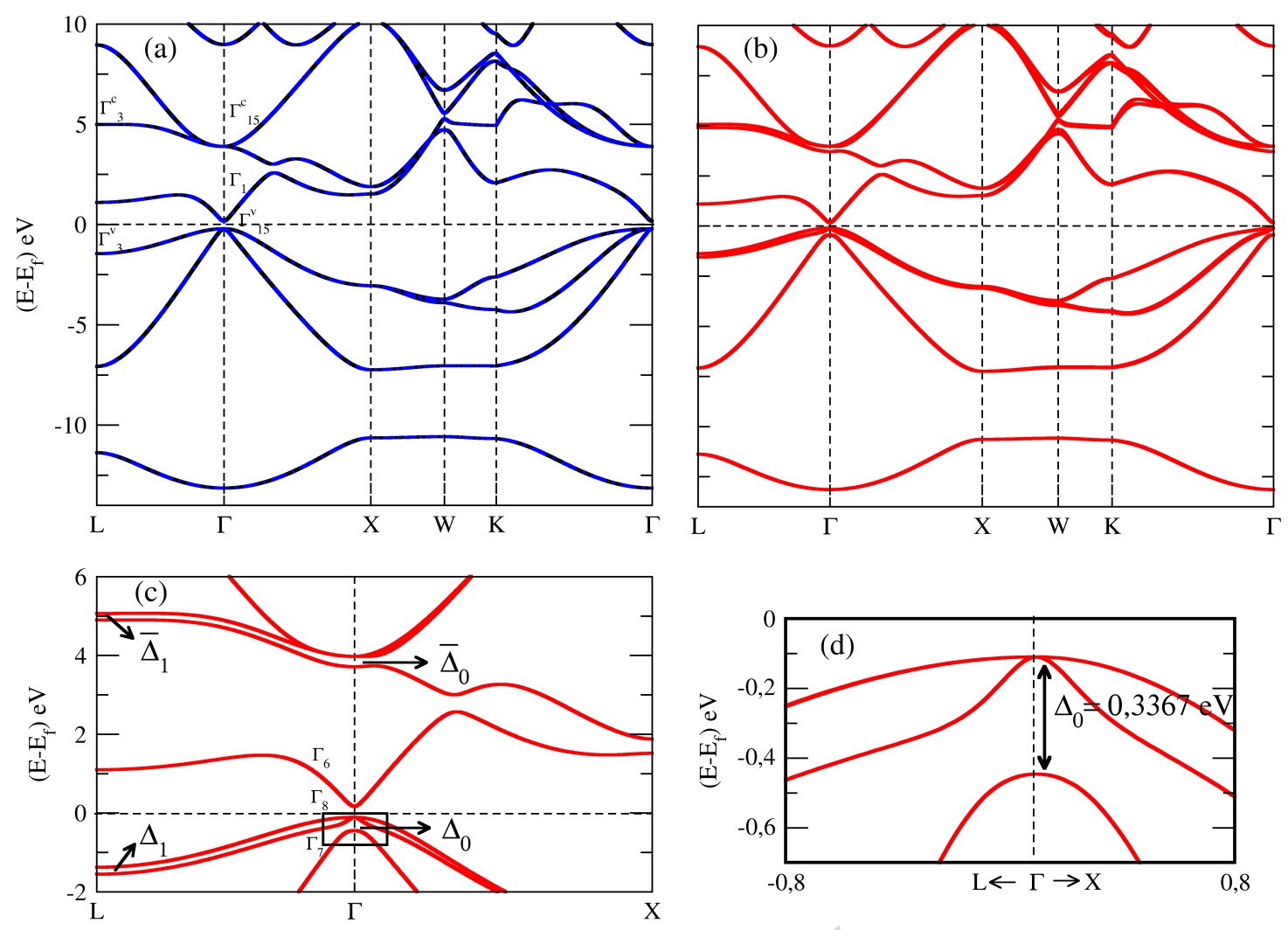

Figura 5.1: Estrutura de bandas do GaAs. (a) Estrutura de bandas sem levar em consideração o SOC. (b) Estrutura de bandas considerando o SOC. (c) Efeitos do SOC. (d) Zoom no retângulo preto da figura (c) que evidência o valor de $\Delta_{0}$.

Férnandez-Seivane et al.[24], mostraram que os sistemas estudados mediante o formalismo do pseudopotencial relativístico de norma conservada implementado no código SIESTA dentro da aproximação "on-site" do SOC atingem valores quase constantes na energia total, gap de energia e gap SOC para bases do tamanho da dupla-Z polarizada e superiores. Os valores da energia total e gap SOC para o GaAs são apresentados na tabela 5.2. Consequentemente, em todos os cálculos feitos neste trabalho consideraram-se tamanhos de bases no mínimo iguais à base DZP. Com esta base o SIESTA-SOC apresenta uma profunda concordância com outros códigos e dados experimentais na descrição da estrutura de bandas de sistemas periódicos.

O controle da força da interação spin-órbita pode ser uma ferramenta 

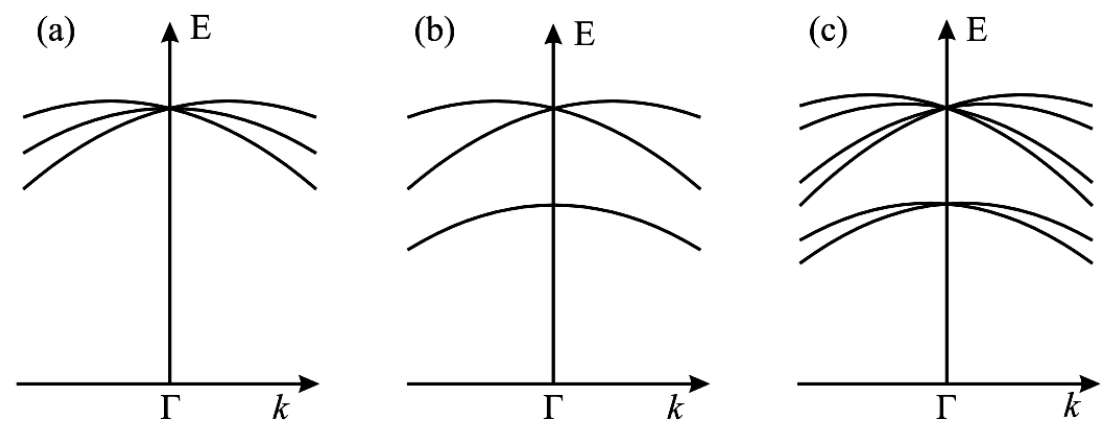

Figura 5.2: Esquema da mudança da estrutura de bandas no ponto $\Gamma$ em sistemas com simetria $T_{d}$. Quando se considera uma única espécie atômica o sistema possui simetria $O_{h}$, sem levar em consideração o SOC, apresenta um estado oito vezes degenerado no ponto $\Gamma$, de forma que a estrutura de bandas apresenta o formato do desenho pictórico em (a). Entretanto quando se considera o SOC o quebra-se a degenerescência dos estados de caráter $p$ em $j=1 / 2$ e $j=3 / 2$ como representado em (b), se o sistema possui dos átomos de espécies diferentes a degenerescência de spin e levantada fora do ponto $\Gamma$ como é esquematizado em (c).

Tabela 5.1: Valores dos gap SOC em eV calculados usando o SIESTA-SOC comparados com os valores experimentais.

\begin{tabular}{|c|c|c|c|c|}
\hline & $\Delta_{0}$ & $\bar{\Delta}_{0}$ & $\Delta_{1}$ & $\bar{\Delta}_{1}$ \\
\hline \hline SIESTA-SOC & 0.3367 & 0.2624 & 0.1733 & 0.1666 \\
\hline Experimental[29] & 0.34 & 0.26 & 0.23 & 0.11 \\
\hline Ref[24] & 0.3573 & 0.3006 & 0.1857 & 0.319 \\
\hline
\end{tabular}

de grande utilidade para o estudo das propriedades dos sistemas em casos limites. Esta ferramenta foi implementada neste trabalho. Considerando a contribuição da correção associada ao SOC por átomo, permite-se ao usuário definir um múltiplo da contribuição do SOC por espécie atômica no cálculo. Esta ferramenta será aplicada no estudo de efeitos de atual interesse na física da matéria condensada. Entretanto, para apresentar seu funcionamento pode-se observar a variação do valor do gap SOC $\Delta_{0}$ no GaAs para diferentes valores do SOC até atingir o valor real. Embora o gap SOC no GaAs tenha sido estudado tanto experimentalmente quanto teoricamente, a análise apresentada pode ser considerada como uma demonstração da relação do gap SOC com a variação exclusivamente do SOC.

Note-se que a variação do Gap SOC para valores pequenos do SOC é linear, porém com o aumento do SOC a dispersão muda mantendo a posição e caráter 
Tabela 5.2: Valores dos gap SOC em eV calculados usando o SIESTA-SOC com diferentes tamanhos da base.

\begin{tabular}{|c|c|c|c|c|}
\hline & $\Delta_{0}$ & $\bar{\Delta}_{0}$ & $\Delta_{1}$ & $\bar{\Delta}_{1}$ \\
\hline \hline SZ & 0.2851 & 0.1956 & 0.1485 & 0.1382 \\
\hline DZ & 0.315 & 0.235 & 0.1572 & 0.1648 \\
\hline DZP & 0.3367 & 0.2624 & 0.1733 & 0.1666 \\
\hline TZP & 0.3314 & 0.2590 & 0.1742 & 0.1668 \\
\hline
\end{tabular}

(a)
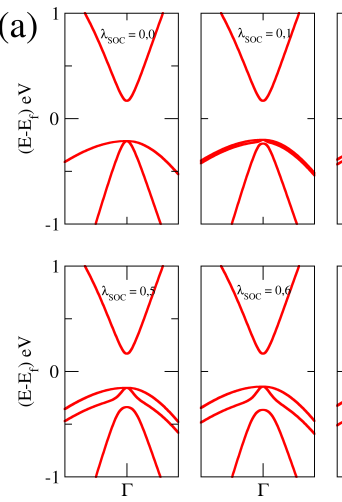
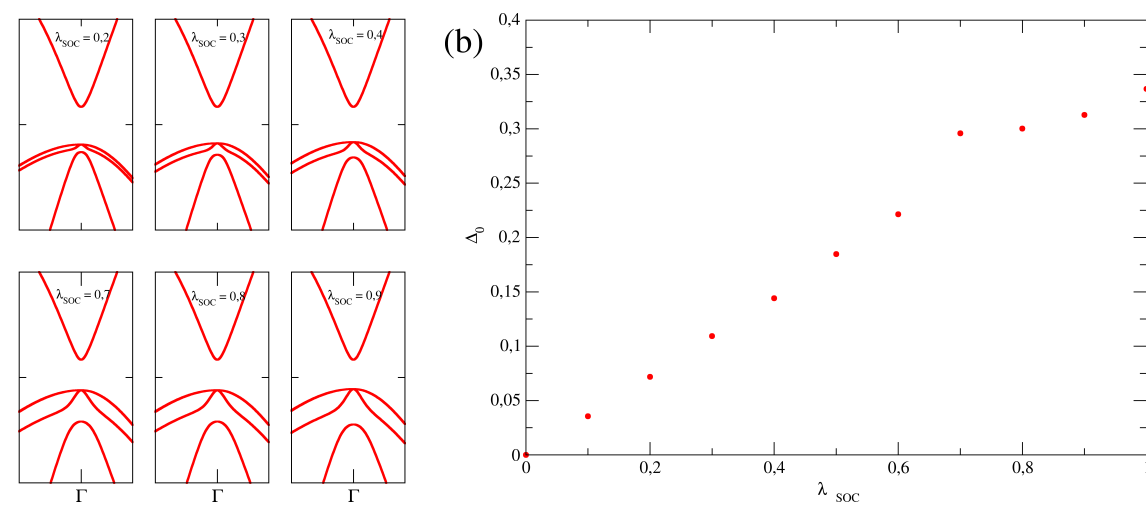

Figura 5.3: Variação do $\Delta_{0}$ em relação à força do SOC no GaAs. (a) Estrutura de bandas para diferentes valores múltiplos do SOC, $\lambda_{S O C}$. (b) Comportamento o valor de $\Delta_{0}$ para diferentes valores de $\lambda_{S O C}$.

dos níveis. Existe um conjunto de materiais caracterizados por uma inversão na "ordem" dos níveis associada com a presença do SOC. Recentemente estes materiais têm chamado a atenção, dado que permitem a observação de fases topológicas na matéria[4,5]. Em particular, neste trabalho estudamos um sistema com inversão de banda gerada pelo SOC conhecido como Telureto de Mercúrio, HgTe.

\subsection{Efeito Hall Quântico de Spin}

A física da matéria condensada prévia aos anos oitenta está fundamentada na teoria microscópica de muitos corpos e na teoria fenomenológica de GinzburgLandau, a qual tem como conceito fundamental o "princípio de quebra de simetria". Este princípio associa-se com a possibilidade de classificar as fases estáveis da 
matéria de acordo às simetrias que elas quebram ${ }^{1}$. Consequentemente, na física da matéria condensada, uma das formas de estudar as fases estáveis da matéria sólida é classificando-as de acordo aos grupos espaciais de simetria.

$\mathrm{Na}$ teoria de Ginzburg-Landau[30] uma fase estável da matéria é caracterizada por uma parâmetro de ordem local. Nas fases desordenadas o parâmetro de ordem local é nulo enquanto que as fases ordenadas, além de ser caracterizadas por parâmetros de ordem diferentes de zero, distinguem-se pela forma em que este transforma-se sob operações de simetria ${ }^{2}$. Entretanto, no ano 1980 o experimento de Klitzing et al.[31], evidenciou a existência de uma fase estável da matéria que não pode ser caracterizada por um parâmetro de ordem local, esta fase denominou-se efeito Hall Quântico (QH).

O efeito QH observa-se quando um gás de elétrons bidimensional (2DEG) está sob a ação de um campo magnético forte perpendicular ao plano que o contêm, provocando que os elétrons se desloquem através de órbitas circulares denominadas órbitas ciclotrônicas. O raio destas torna-se menor com o aumento do campo magnético. Para campos magnéticos muito fortes, os elétrons do "bulk" do material formam órbitas ciclotrônicas pequenas e fechadas. E os elétrons próximos da borda podem traçar extensas órbitas abertas pulando ao longo da borda. A baixas temperaturas, a área das órbitas fechadas no "bulk" torna-se quantizada, os elétrons do "bulk" ficam localizados e o "bulk" transforma-se em um isolante. Adicionalmente, os orbitais dos elétrons na borda formam canais unidimensionais onde cada canal apresenta uma condutância de $e^{2} / \hbar$, desta forma a condutância está quantizada em termos do número de canais. Por outra parte, a condutância transversal $\sigma_{x y}$ está quantizada em inteiros múltiplos de $e^{2} / \hbar$.

O "bulk" de um estado QH não quebra nenhuma simetria além da simetria

\footnotetext{
${ }^{1}$ Por exemplo, os sólidos cristalinos quebram a simetria translacional de forma que obedecem a um subgrupo discreto de simetrias translacionais e rotacionais denominado grupo espacial.

${ }^{2}$ Como pela representação do grupo de simetria do hamiltoniano do sistema.
} 
de inversão temporal, a qual não está caracterizada por um parâmetro de ordem local. No entanto, os estados QH com diferentes valores de condutância Hall correspondem a estados quânticos que não podem estar conectados adiabaticamente uns com os outros sem fechar um gap espectral. Além do mais, a quantização da condutância Hall existe inclusive em amostras desordenadas onde se espera que o efeito da aleatoriedade da desordem destrua o fenômeno de quantização.

A falta da descrição de um parâmetro de ordem local para o "bulk" de um QH na teoria de Ginzburg-Landau assim como a existência de estados de borda robustos à desordem podem ser entendidos como características definidas de um outro estado topológico da matéria.

Um estado topológico da matéria é isolante no "bulk" com estados metálicos na borda que são perturbativamente robustos à desordem. Ao invés de ser caracterizado por um parâmetro de ordem local, o "bulk" está caracterizado por um invariante topológico que no caso de um estado QH é um inteiro denotado como número Chern, dado pela expressão:

$$
C=\frac{1}{2 \pi} \sum_{n} \int_{B Z} d^{2} k \Omega_{n}
$$

onde $\Omega_{n}$ é a curvatura de Berry momento-espaço para a banda $n$ que escreve-se como[32,33,34]:

$$
\Omega_{n}(k)=-\sum_{n^{\prime} \neq n} \frac{2 \operatorname{Im}\left\langle\psi_{n k}\left|v_{x}\right| \psi_{n^{\prime} k}\right\rangle\left\langle\psi_{n^{\prime} k}\left|v_{y}\right| \psi_{n k}\right\rangle}{\left(\omega_{n^{\prime}}-\omega_{n}\right)^{2}}
$$

sendo a soma sobre todas as bandas ocupadas abaixo do gap do "bulk", $\omega_{n} \equiv E_{n} / \hbar$, e $v_{x(y)}$ o operador de velocidade.

O número de Chern é um invariante topológico do "bulk" isolante relacionado ao número de estados metálicos estáveis na borda. No estado QH inteiro, o número Chern é igual ao número de estados metálicos estáveis na borda e também 
ao valor da condutância Hall quantizada em unidades de $e^{2} / h$. Neste sentido os estados da borda estariam protegidos pela topologia do "bulk" a qual é responsável por alguns tipos de fracionamento (quebra de simetria espacial) na borda. Mais precisamente, os graus de liberdade do elétron estariam separados espacialmente em bordas opostas. Usualmente um elétron em um canal unidimensional pode-se movimentar nos dois sentidos. Porém, na borda de uma amostra QH os elétrons movimentam-se em um único sentido o qual depende do sinal do campo magnético. Portanto, os canais da borda de QH são denominados quirais. O retroespalhamento na borda é suprimido devido à inabilidade do elétron para reverter sua direção de movimento e consequentemente os canais da borda evadem completamente a localização de Anderson.

Os estados QH foram considerados os únicos estados topológicos observáveis experimentalmente. Não obstante a pesquisa da existência de outros estados topológicos que não precisam de campo magnético externo permitiu a predição teórica[41] e posterior descoberta experimental[5] em poços quânticos de HgTe do efeito Hall quântico de spin (QSH). Este novo estado topológico da matéria apresenta algumas similaridades e diferenças fundamentais com os estados QH[77].

\subsubsection{Fenomenologia do efeito QSH}

O efeito Hall de carga leva de forma natural ao efeito QH. Assim, a descoberta experimental do efeito Hall de Spin (HS) [35,36,37,38] induzido pelo SOC em semicondutores dopados pode levar a pensar na existência do análogo quântico

deste efeito. Kane e Mele[3] previram teoricamente que o grafeno apresenta o denominado efeito Hall Quântico de Spin (QSH). A proposta foi matematicamente motivada pelo trabalho de Haldane[39] no chamado efeito Hall quântico anômalo (QHA) em grafeno. No entanto, devido à pequena interação spin-órbita intrínseca do carbono não tem sido possível observar estados QSH no sistema proposto por 
Kane e Male[3].

No efeito HS cria-se um fluxo de corrente transversal de spin, por exemplo na direção $x$ quando é aplicado um campo elétrico na direção $y$. Em contraste com o efeito hall, o qual quebra a simetria de reversão temporal (TR) devido ao campo magnético aplicado, o efeito HS não quebra a simetria de TR. Isto pode ser explicado já que no efeito Hall a corrente transversal é dada por $J_{x}=\delta_{x y} E_{y}$, onde $\delta_{x y}$ é a condutividade Hall e $E_{y}$ é o campo elétrico. A presença do campo magnético no efeito Hall permite a presença de uma corrente $\delta_{x y} \neq 0$ quantizada e quebra a simetria de TR. Por outro lado, a corrente HS é dada por, $J_{x}^{s}=\delta_{x y}^{s} E_{y}$, onde $\delta_{x y}^{s}$ é a condutividade HS. Consequentemente, dado que $\delta_{x y}=0$ é consistente com uma simetria de TR, em contraste com a corrente de carga $J_{x}$, a corrente de spin $J_{x}^{s}$ é um invariante do operador de simetria de TR, ou seja, preserva a simetria de TR. O papel do campo magnético no efeito QH é adotado pelo acoplamento spin-órbita da estrutura de bandas no efeito QSH.
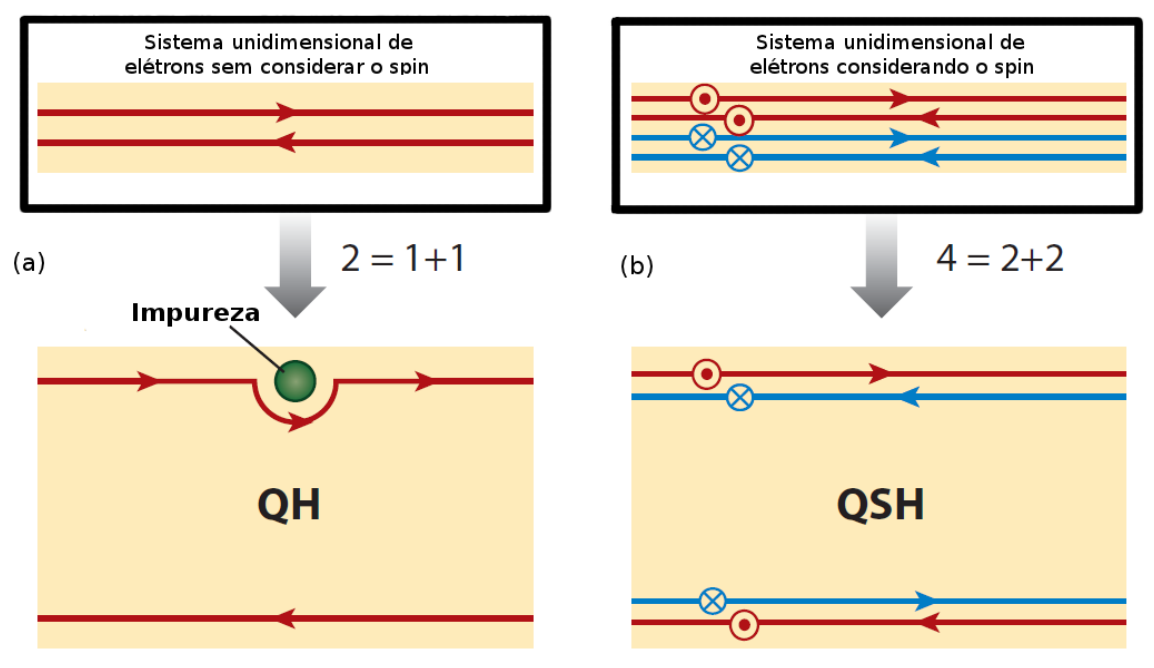

Figura 5.4: Comparação dos estados de borda em sistemas que apresentam o efeito QH e QSH. (a) Na parte superior são esquematizados os possíveis sentidos de movimento de um elétron em um canal unidimensional sem considerar o spin. Na parte inferior é esquematizado o comportamento dos estados de borda presentes no efeito QH, as setas indicam a direção de propagação da corrente de carga. (b) Na parte superior são esquematizadas os possíveis sentidos de movimento de um elétron em um canal unidimensional considerando o spin. Na parte inferior é esquematizado o comportamento dos estados de borda no efeito QSH, as setas indicam o sentido de propagação da corrente de spin. 
Para entender a formação de estados topológicos no efeito QSH é necessário compreender porque o QSH é um estado topológico da matéria e qual é o mecanismo de quantização neste efeito. A teorização dos estados QSH inicia-se com a análise da presença de estados metálicos estáveis robustos à desordem na borda e à comparação com o efeito QH de carga. Dessa forma, deve-se partir da fenomenologia dos estados QH. Estes últimos comportam-se como um isolante no "bulk" mas suportam estados metálicos na borda robustos à desordem. Os estados de gap nulo são tais que os elétrons podem se propagar em uma única direção de uma borda dada. Comparado com um sistema unidimensional de elétrons, sem considerar o spin, representado na parte superior da figura 5.4a, a borda superior dos sistemas QH contém só a metade dos graus de liberdade, como esquematizado na parte inferior da figura 5.4a. O sistema QH por conseguinte, pode-se comparar com uma "via livre" onde os elétrons que se deslocam em direções opostas têm que "dirigir em diferentes pistas". Esta separação espacial resulta em canais quirais que podem ser ilustrados pela equação $2=1+1$, onde cada 1 corresponde a uma quiralidade diferente. Esta regra do "tráfico quiral" é efetiva particularmente em suprimir o retroespalhamento de elétrons. Como os elétrons sempre se deslocam na mesma direção estão forçados a "evitar" impurezas e deste modo são livres de retroespalhamento, como representado na figura 5.4a.

Por outro lado, o estado QSH poderia ser entendido como duas cópias do estado QH como ilustrado na figura 5.5, uma para cada spin, ou seja, a estrutura do estado da borda do estado QSH pode ser descrita de forma pictórica pela superposição de duas cópias do estado de borda QH, com quiralidade oposta para cada spin. Comparado com um sistema unidimensional onde se considera o spin, representado na parte superior da figura 5.4b, a borda superior do sistema QSH contém só a metade de graus de liberdade e o estado resultante da borda é denominado helicoidal já que o spin está correlacionado com a direção de propagação como é representado na parte inferior da figura 5.4b. Este novo padrão de separação 


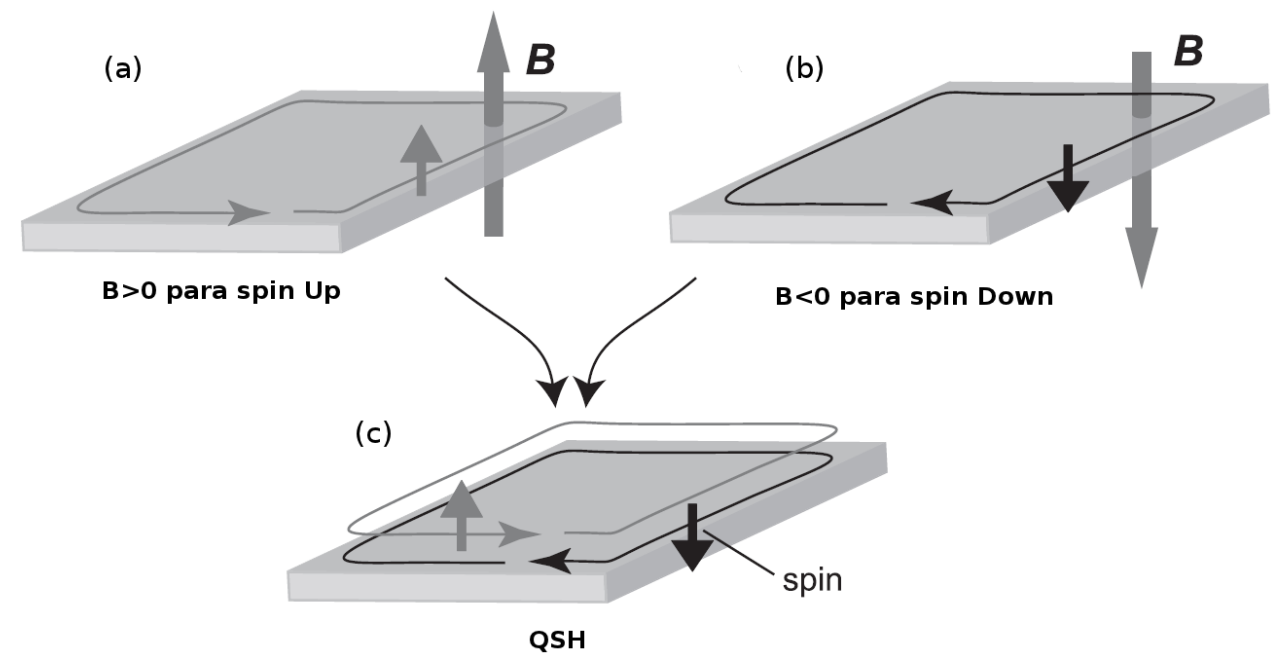

Figura 5.5: Formação do QSH a partir de duas copias do estado QH. Em (a) e (b) se apresentam duas amostras QH com campos magnéticos aplicados em sentidos opostos, de forma que exitem uma amostra QH para cada componente de spin. A "interseção" deste sistemas fornece um sistema com dos canais de condução com correntes de spin diferente se propagando em sentidos opostos. De modo que as duas copias do QH resultam em um sistema com as caraterísticas do efeito QSH como apresentado em (c).

espacial pode ser ilustrado simbolicamente pela equação $4=2+2$, onde cada 2 corresponde a diferente helicidade. Portanto, os elétrons podem "viajar" para a frente e para trás na mesma borda com somente uma componente de spin em cada sentido do movimento, o que será a nova "regra de trânsito" que elimina o retroespalhamento. Para retroespalhar um elétron é necessário modificar a orientação do spin, o qual requer da ruptura da simetria TR. Desta forma, se a simetria TR é preservada, elimina-se completamente o retroespalhamento, este mecanismo de proteção é denominado topológico. Por exemplo, dado que impurezas não magnéticas preservam a simetria de TR, uma amostra QSH com estas impurezas apresenta estados topológicos livres de retroespalhamento.

A separação espacial no caso do efeito QH deve-se à presença de um campo magnético externo e no caso do efeito Hall Quântico Anômalo (QHA) a campos internos os quais quebram a simetria TR. Estes campos internos tomam a forma de um termo de massa relativística para férmions de Dirac emergentes em dimensões 
$2+1^{3}$. No caso do efeito QSH, a separação é atingida através do SOC.

Por causa do estado QSH estar caracterizado pela presença de estados de "bulk" isolantes e estados de fronteira metálicos robustos à desordem (na presença de simetria TR), o estado QSH é certamente um novo estado topológico da matéria. Não obstante, já que no estado QSH a condutância Hall é nula, o número Chern ou TKNN, o qual corresponde ao valor da condutância Hall em unidades de $e^{2} / h$ não pode proporcionar uma classificação adequada para esta nova fase topológica da matéria. Esta questão tem sido abordada dentro da teoria de bandas topológicas e a teoria de campos descritas por Qi, Hughes e Zhang[40]. Encontrou-se que o invariante topológico apropriado e avaliado no grupo $Z_{2}$ o qual contem só dois elementos, 0 ou 1, com 1 correspondendo ao isolante topológico não trivial, ou seja, um QSH, e 0 correspondendo ao isolante trivial com estados de borda não robustos. Fisicamente, o invariante $Z_{2}$ conta o número de estados metálicos estáveis na borda.

A procura de sistemas com estados metálicos protegidos topologicamente pela simetria de TR, tem-se convertido em um importante foco de pesquisa na física da matéria condensada. A primeira observação experimental de estados QSH foi classificada pela revista Science como sendo um dos 10 trabalhos mais importantes do ano 2007 na física da matéria condensada. Esta comprovação experimental do efeito QSH parte do estudo de uma heteroestrutura formada por $\mathrm{CdTe} / \mathrm{HgTe} / \mathrm{CdTe}$. Os autores foram motivados pela predição teórica de estados QSH em poços quânticos de semicondutores com inversão de banda induzida pelo SOC[41] tal como o HgTe [5,42]. Para entender a particular estrutura de bandas do HgTe, que permite a formação deste fenômeno, é necessário o entendimento da estrutura eletrônica e inversão de banda deste sistema.

\footnotetext{
${ }^{3} \mathrm{O}$ sinal do campo interno é ditado pelo signo da massa
} 


\subsubsection{Inversão de banda e estrutura eletrônica do $\mathrm{HgTe}$}

Groves et al.[43], mostraram a existência de um fenômeno no HgTe denominado inversão de banda. Os materiais que apresentam este particular comportamento têm sido classificados como "sistemas de banda invertida". Nos semicondutores, usualmente o mínimo da banda de condução $\Gamma_{6}$ com caráter $s$ situa-se acima do máximo da banda de valência $\Gamma_{8}$ com caráter $p$, separados por um gap de energia positivo definido como $E_{0}=E\left(\Gamma_{6}\right)-E\left(\Gamma_{8}\right)$, como foi mostrado na seção anterior. No entanto, o HgTe fornece um valor de $E_{0}$ negativo dado que o forte SOC inverte a "ordem" dos estados caraterizados como $\Gamma_{6}$ e $\Gamma_{8}$. O mecanismo de inversão de banda não é trivial, porém nos semicondutores com um forte SOC pode-se entender este mecanismo fenomenologicamente. Nas bandas que possuem unicamente contribuições dos orbitais $s$ tal como $\Gamma_{6}$ os efeitos do SOC são nulos, de modo que ao incluir os SOC, estas bandas não são perturbadas. O SOC perturba as bandas de caráter $p$ corrigindo energeticamente sua posição no espectro de energias. Deste modo, na estrutura de bandas dos semicondutores, além de aparecer a separação do topo da banda de valência em $\Gamma_{7}$ e $\Gamma_{8}$, gera-se um "deslocamento" energético desta bandas em relação a $\Gamma_{6}$. Isto implica que a ordem das bandas $\Gamma_{6}, \Gamma_{7}, \Gamma_{8}$ muda na presença de um SOC suficientemente forte, ou seja, se a contribuição do SOC é positiva e muito forte é possível obter uma ordem energética das bandas do topo da banda de valência como $\Gamma_{8}, \Gamma_{7}, \Gamma_{6}$ ou no caso do HgTe na ordem: $\Gamma_{8}, \Gamma_{6}, \Gamma_{7}$.

A inversão de banda ocasionada pelo SOC, no caso particular do HgTe gera um gap SOC $\Delta_{0}$ maior que o gap $E_{0}$ de forma que a banda $\Gamma_{6}$ situa-se entre as bandas $\Gamma_{7}$ e $\Gamma_{8}$ separadas pelo gap SOC $\Delta_{0}$. Este comportamento não é observado em outros semicondutores com inversão de banda como o HgS e HgSe. Desta forma a comparação da estrutura de banda dos semicondutores CdTe e HgTe resulta na visualização da mudança da posição da banda $\Gamma_{6}$ em relação às bandas $\Gamma_{8}$ e $\Gamma_{7}$ como 
ilustrado na figura 5.6a.
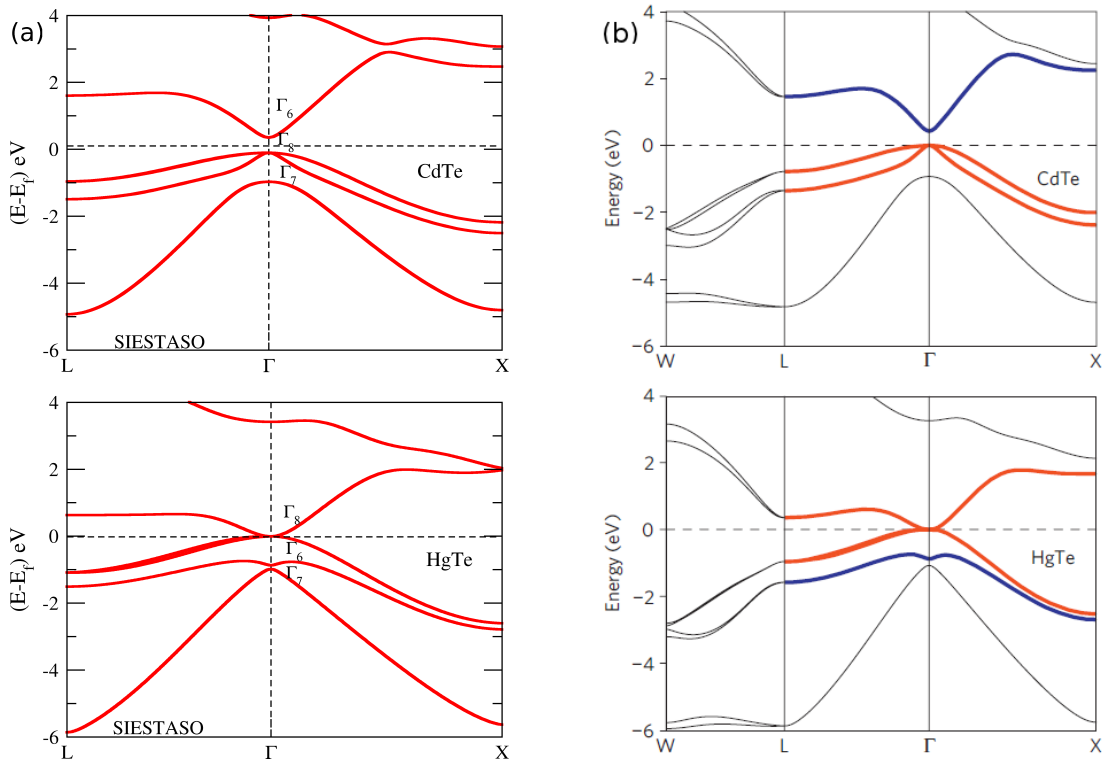

Figura 5.6: Estrutura de bandas do HgTe e CdTe. (a) Cálculos feitos com a implementação do SOC no código SIESTA. (b) Cálculos de Stanislav Chadov et al. (2012), a cor vermelha representa os estado de caráter $p\left(\Gamma_{8}\right)$ e a cor azul os estados de caráter $s\left(\Gamma_{6}\right)$.

No presente estudo, observou-se uma concordância na estrutura de bandas obtidas por Chadov et al.[44], e a feita com o SIESTA-SOC. É importante esclarecer que além da necessidade da inclusão do SOC para a descrição do HgTe, esta interação relativística deve ter o valor numérico adequado no cálculo para obter a ordem certa das contribuições associadas aos orbitais $p$ e $s$ na estrutura de bandas. Adicionalmente, a particular disposição destes estados no HgTe em relação ao CdTe permite a observação do QSH em uma interface formada por estes materiais. A explicação do processo de "conexão" destes materiais e a formação de estados QSH abrange a conceitualização do efeito QSH já estudada e análise do poço quântico de $\mathrm{HgTe} / \mathrm{CdTe}$.

\subsubsection{Poços quântico em $\mathrm{HgTe} / \mathrm{CdTe}$}

O HgTe apresenta estados metálicos, ou seja, comporta-se como um semicondutor de gap nulo com os estados $\Gamma_{6}$ e $\Gamma_{8}$ trocados em relação ao 
CdTe. Consequentemente, em um poço quântico de HgTe/CdTe, a partir de uma determinada espessura $d_{c}$ o HgTe apresenta um gap e portanto sua conexão com o CdTe somente pode ser via um estado metálico protegido. Em outras palavras, para um comprimento $d<d_{c}$, o CdTe "impõe" seu comportamento de forma que os estados $E_{1}\left(\Gamma_{6}\right)$ e $H_{1}\left(\Gamma_{8}\right)$ da heteroestrutura possuem a mesma ordem deste material como é mostrado na figura 5.7c. Entretanto, quando se considera um poço quântico com uma espessura $d>d_{c}$, o HgTe impõe seu comportamento de forma que o SOC é suficientemente forte para gerar uma mudança na ordem destes níveis resultando a configuração da figura 5.7c. Em consequência, a heteroestrutura apresenta um cone de Dirac em cada interface resultado do cruzamento dos estados $E_{1}$ e $H_{1}$ na forma representada na figura 5.7b. Quando a largura do poço quântico atinge $6.3 \mathrm{~nm}$, a posição dos níveis muda obedecendo ao gráfico da figura 5.7a no qual é observado o cruzamento na largura mencionada. Com o experimento de 4terminais, Köing et al.[5], verificaram este comportamento. Este experimento é visto como a comprovação experimental da existência dos estados QSH.

Embora a predição teórica de estados QSH em HgTe/CdTe permitiu sua descoberta experimental, de acordo com Lou e Zunger[45], o desenvolvimento inicialmente proposto por Bernevig, et al.[41], não é adequado, dado que os estados da base usados no método $\boldsymbol{k} \cdot \boldsymbol{p}$ não representavam o "bulk" do sistema. Consequentemente, os estados de superfície representados nesta aproximação não estavam de acordo com o limite no qual as duas interfaces do poço quântico são equivalentes[46]. Lou e Zunger[45] propuseram um modelo que em princípio fornece uma descrição adequada dos estados de interface deste sistema. O modelo baseado na teoria de perturbação de bandas de energia inclui todas as contribuições da banda $\Gamma_{8}$, HH e LH (heavy hole e light hole as duas associadas a bandas de carater tipo $p$ ), de forma a obter um cruzamento para cada interface da heteroestrutura resultando a estrutura de bandas da figura 5.7d. 


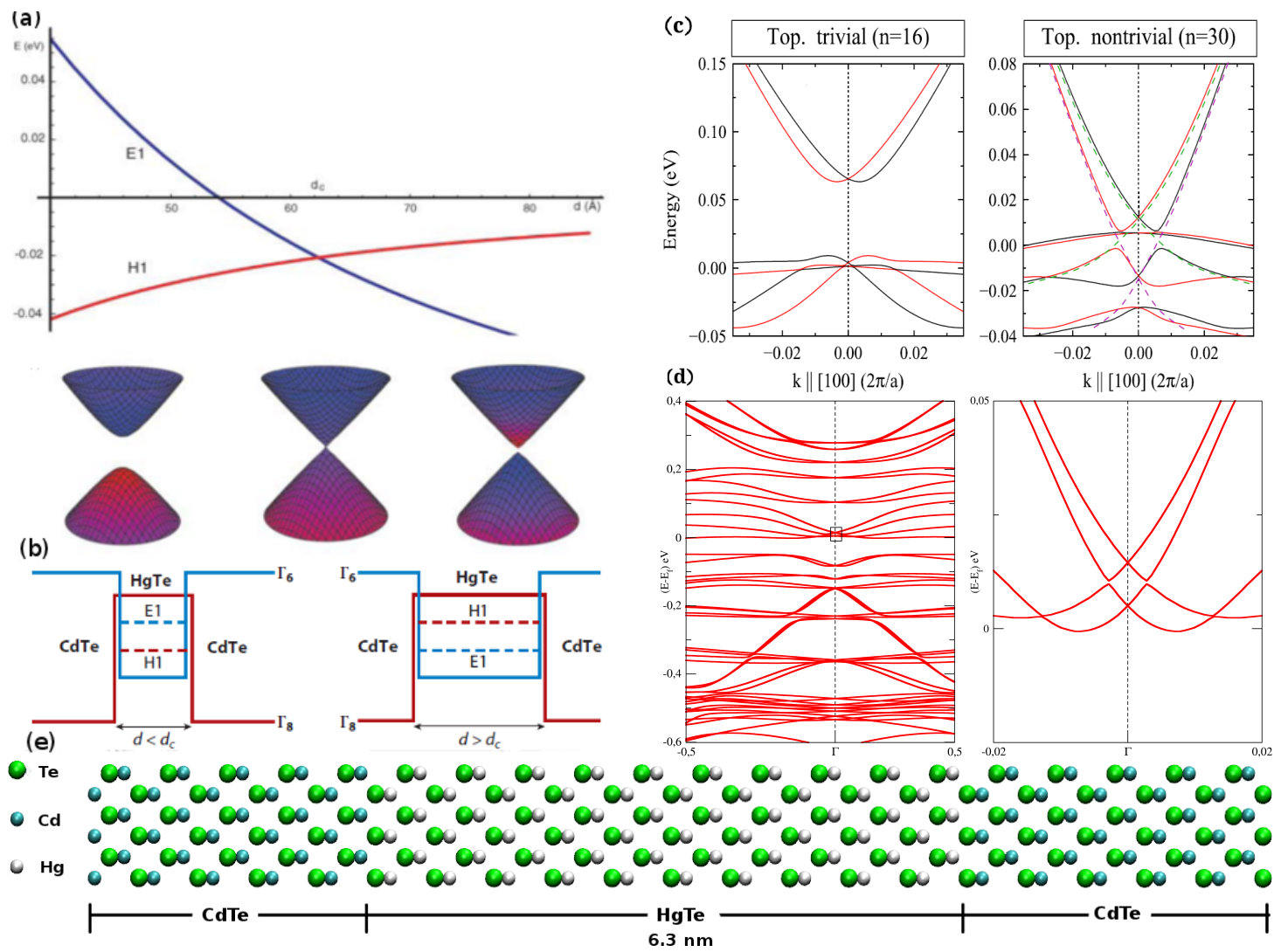

Figura 5.7: Estados de interface no poço quântico de $\mathrm{HgTe} / \mathrm{CdTe}$.(a) Evolução dos níveis de energia $E_{1}$ (Azul) e $H_{1}$ (vermelho) em relação à largura, $d$, do HgTe. Embaixo se apresenta a relação de dispersão para $d=40,63$ e $70 \mathrm{Ang}$, sendo $d_{c}=6.3 \mathrm{~nm}$ a largura na qual forma-se o cone de Dirac. (b) Desenho esquemático da ordem dos níveis $E_{1}$ (Azul) e $H_{1}$ (vermelho) no poço quântico de $\mathrm{HgTe} / \mathrm{CdTE}$, antes e depois do cruzamento. (c) Estrutura de bandas nas proximidade do nível de Fermi obtida por Luo e Zunger[45], sendo $n$ o número de monocamadas de $\mathrm{HgTe}$. Quando $\mathrm{n}=16$ o sistemas não apresenta cruzamento dos níveis $E_{1}$ e $H_{1}$. Enquanto que, para $\mathrm{n}=30$ o sistema apresenta o QSH devido ao cruzamento desses níveis. (d) Na esquerda, a estrutura de bandas usando o SIESTA-SOC. Na direita, zoom no retângulo nas proximidades do nível de Fermi. (e) Desenho pictórico do sistema.

A implementação desenvolvida neste trabalho consegue reproduzir os resultados do trabalho de Lou e Zunger[45] como apresenta-se na figura 5.7e. O sistema usado, ilustrado na figura $5.7 f$ possui a largura crítica reportada. Na literatura não há indícios de cálculos feitos para este sistema usando DFT já que o sistema é muito grande e as atuais implementações do SOC requerem bastante tempo para fazer este tipo de simulações.

O estado QSH foi previsto em vários materiais[41,47]. Embora experi- 
mentos conseguiram observar esta fase topológica em poços quânticos de $\mathrm{HgTe}[5]$, a atividade experimental em relação ao efeito QSH permaneceu limitada pelas dificuldades práticas associadas às estruturas baseadas neste material. Entretanto, tem-se descoberto sistemas 3D que apresentam estados topológicos e permitem sua manipulação experimental. Neste trabalho foi estudada a formação dos estados topológicos nestes sistemas 3D.

\subsection{Isolantes topológicos}

Os estados QSH apresenta uma "generalização natural" em três dimensiones $[48,49,50]$ chamada isolante topológico (TI). De acordo com a descrição de Moore and Balents[49], estes sistemas 3D apresentam um gap no "bulk" (da mesma forma que um isolante comum) com estados eletrônicos metálicos na superfície na forma de um cone de Dirac, os estados metálicos são protegidos pela simetria de reversão temporal, e consequentemente, não podem ser destruídos por perturbações tais como impurezas não magnéticas ou imperfeições da rede. A presença desta robustez à desordem e à presença de impurezas não magnéticas tem sido prevista em vários materiais[4]. Entretanto, o primeiro TI descoberto experimentalmente foi o $\mathrm{Bi}_{1-x} \mathrm{Sb}_{x}[6]$. Este material é considerado como o representante de uma família de materiais tais como: $\mathrm{Bi}_{2} \mathrm{Se}_{3}, \mathrm{Bi}_{2} \mathrm{Te}_{3}$ e $\mathrm{Sb}_{2} \mathrm{Te}_{3}$. Contudo, embora seja pensado nos TI como uma generalização do efeito QSH, existe uma diferença fundamental na descrição destas fases topológicas. Enquanto que os estados QSH são caracterizados pelo invariante topológico $\mathrm{Z}_{2}$, os TI são caracterizados por quatro invariantes topológicos $\mathrm{Z}_{2}:\left(v_{0}, v_{1}, v_{2}, v_{3}\right)[50]$, porém para entender o papel do SOC e a simetria TR devem ser estudadas algumas particularidades da formação dos estados topológicos nestes sistemas.

Os estados de superfície de um cristal 3D podem ser rotulados com um 


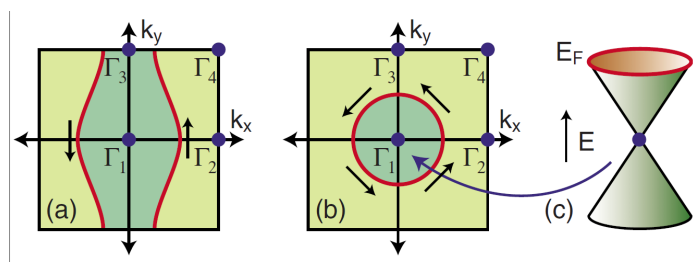

Figura 5.8: Comparação da superfície de Fermi para um isolante topológico fortes e um isolante topológico fraco. Círculos de Fermi na superfície da zona de Brillouin para (a) um isolante topológico fraco e (b) um isolante topológico forte. (c) No isolante topológico forte mais "simples" o círculo de Fermi abrange um único cone de Dirac.

momento cristalino 2D. Por exemplo quando a zona de Brillouin da superfície é quadrada há quatro pontos invariantes do operador de TR $\hat{\Theta}$ denominados $\Gamma_{1,2,3,4}$ como ilustrado na figura 5.8, onde os estados de superfície, se estiverem presentes, devem apresentar degenerescência de Kramer. Longe destes pontos especiais, o SOC levanta a degenerescência e podem-se formar cones de Dirac 2D na estrutura de bandas da superfícies.

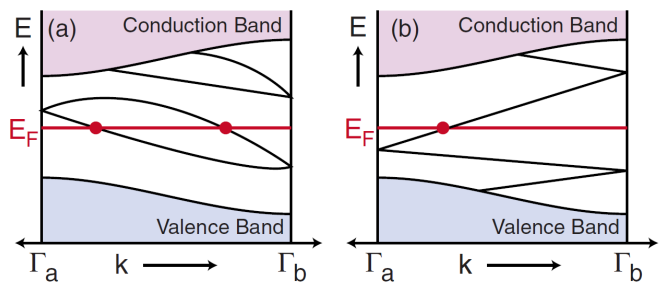

Figura 5.9: Dispersão eletrônica entre dos pontos de superfície Kramers degenerados $\Gamma_{a}=0$ e $\Gamma_{b}=\pi / a$. Em (a) o número de estados de superfície que atravessam a energia de Fermi $E_{F}$ é par e em (b) é impar.

Os estados de Kramer nos diferentes pontos invariantes por TR estão conectados. Especificamente, entre qualquer par $\Gamma_{a}$ e $\Gamma_{b}$, os estados de superfície serão semelhante aos representados na figura 5.9, de modo que no nível de fermi intersecta uma linha que une $\Gamma_{a}$ a $\Gamma_{b}$ um número par ou ímpar de vezes. Se for ímpar os estados de superfície estarão topologicamente protegidos.

Um isolante topológico 3D não trivial pode ser construído empilhando camadas de um QSH 2D. Os estados de borda helicoidal das camadas tornam-se estados de superfície. Uma possível superfície de Fermi para camadas fracamente 
acopladas empilhadas ao longo da direção y é mostrada na figura 5.8a (linha vermelha). Este sistema em camadas é conhecido como um isolante topológico fraco e tem $v_{0}=0$. Os outros índices $\left(v_{1}, v_{2}, v_{3}\right)$ que caracterizam um isolante topológico podem ser interpretados como índices de Miller que descrevem a orientação das camadas. Ao contrário dos estados de borda de um QSH 2D, a simetria TR não protege estes estados de superfície[51].

Na figura 5.8b mostra-se a superfície de Fermi (círculo vermelho) para uma dada energia de Fermi 5.8c. Neste caso, $v_{0}=1$ identifica esta fase chamada isolante topológico forte, que não pode ser interpretado como um "descendente" do QSH (como o isolante topológico fraco). Em consequência, $v_{0}$ determina se o número de cruzamentos é par ou ímpar. Em um isolante topológico forte o "círculo de Fermi" na superfície abrange um número ímpar de pontos de Dirac com degenerescência de Kramer. No caso mais simples há somente um cone de Dirac 5.8b que pode ser descrito pelo hamiltoniano ${ }^{4}$ :

$$
\mathcal{H}_{\text {surface }}=-i \hbar v_{F} \boldsymbol{\sigma} \cdot \nabla
$$

onde $\boldsymbol{\sigma}$ são as matrizes de Pauli e $v_{F}$ a velocidade de Fermi.

Os estados de superfície dos isolantes topológicos fortes formam um único estado metálico topológico, diferentemente de um metal ordinário, os TI tem sua orientação do spin truncada em uma única direção. Visto que a simetria TR requer que os estados com momento $k$ e $-k$ apresentem spin opostos, o spin deve girar em volta com $k$ ao redor da superfície de fermi 5.8b. Em outras palavras, estes materiais além de ter uma superfície sem gap e "bulk" isolante, apresentam elétrons na superfície com uma orientação específica do spin. Esta particularidade o torna benéfico para a eletrônica baseada em spin a qual usa a orientação deste

\footnotetext{
${ }^{4} \mathrm{O}$ hamiltoniano associado a uma dispersão linear, dado que da mesma forma que o grafeno, este sistema também apresenta cones de Dirac.
} 
para armazenar informação. Desta forma, abre-se um mundo de possibilidades nas aplicações tecnológicas na spintrônica.

É de fundamental importância levar em consideração que precisa-se da inclusão do SOC na descrição dos TI, dado que, da mesma forma que nas amostras QSH a inversão de banda gerada pelo SOC determina a formação dos estados metálicos de fronteira que preservam a simetria de reversão temporal, caraterística que define a robustez destes estados. Nessa ordem, o $\mathrm{Bi}_{2} \mathrm{Se}_{3}$ tem sido muito estudado teoricamente já que fornece um entendimento claro da forma como a inversão de banda gerada pelo SOC permite a formação de estados topológicos como é esquematizado na figura 5.10. Consequentemente, neste trabalho foi estudada a formação de estados topológicos na superfície do $\mathrm{Bi}_{2} \mathrm{Se}_{3}$.

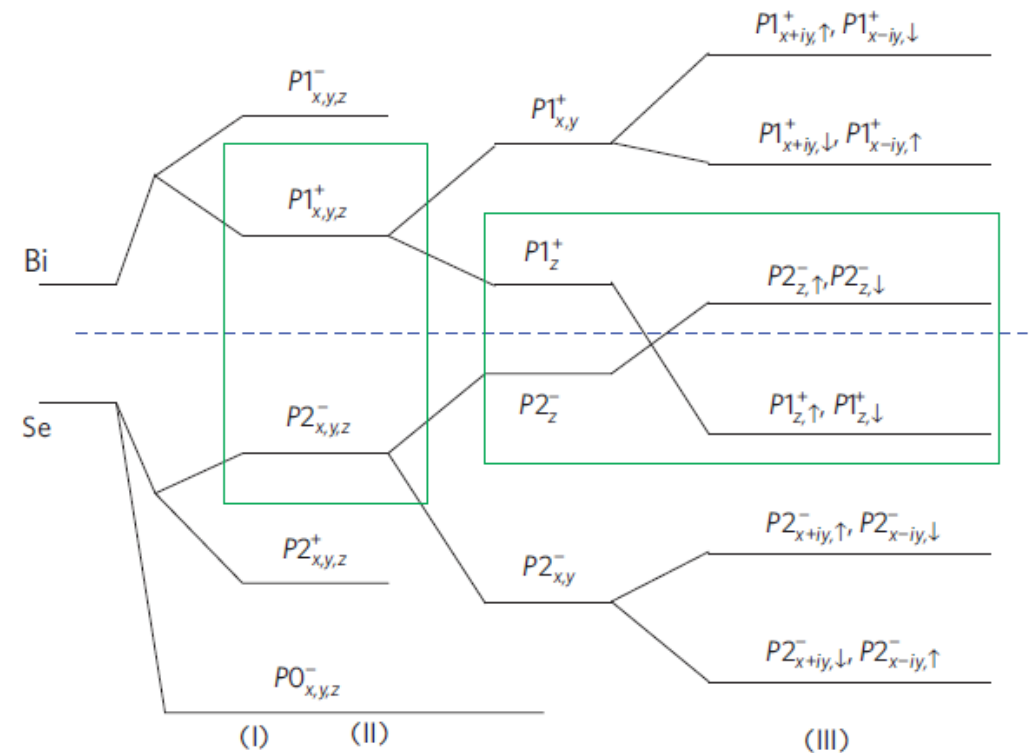

Figura 5.10: Inversão de banda no bulk de $\mathrm{Bi}_{2} \mathrm{Se}_{3}$. A partir dos estados atômicos $P_{x y z}$ do Bi e do Se, pode-se desenhar de forma pictórica a evolução das bandas de valência e condução no ponto $\Gamma$. Os três estágios I, II e II, representam o efeito das ligações químicas, do campo cristalino e do SOC. A variação energética dos níveis que gera o SOC permite a inversão de banda no nível de Fermi. 


\subsubsection{Estados topológicos em $\mathrm{Bi}_{2} \mathrm{Se}_{3}$}

Como foi mencionado o SOC é naturalmente forte para elementos químicos pesados, portanto, estes facilitam a observação de estados topológicamente protegidos pela simetria de reversão temporal. Por conseguinte, um dos primeiros materiais 3D nos quais se observaram estas particulares propriedades foi o $\mathrm{Bi}_{2} \mathrm{Se}_{3}$. Este sistema cristalino tem uma estrutura romboédrica que satisfaz as operações de simetria do grupo espacial $\mathrm{D}_{3 d}$. Contudo, para a observação dos estados topológicos precisa-se gerar uma superfície no sistema e, que o tamanho do sistema seja suficientemente grande para representar as propriedades do "bulk".

a

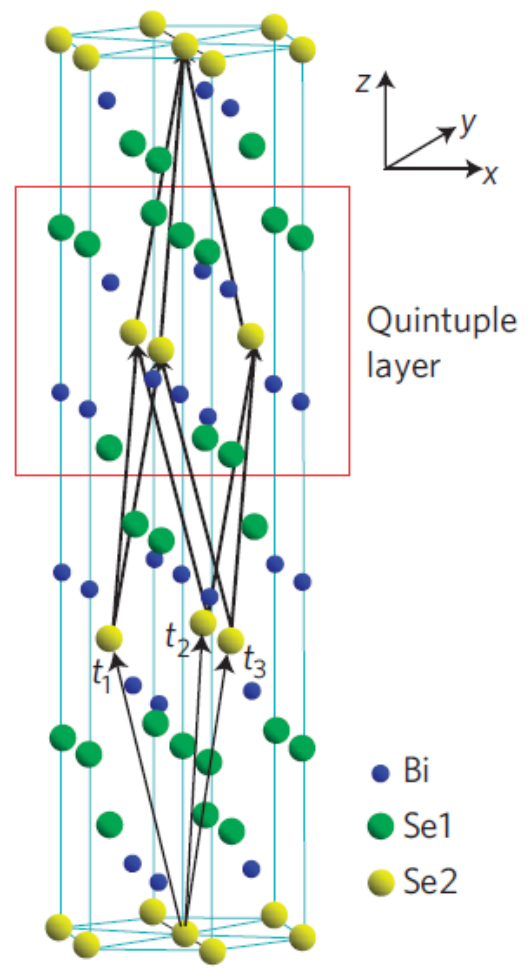

b
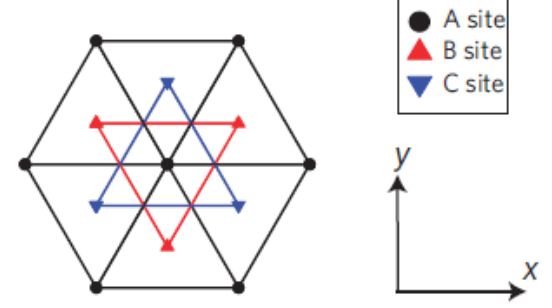

C
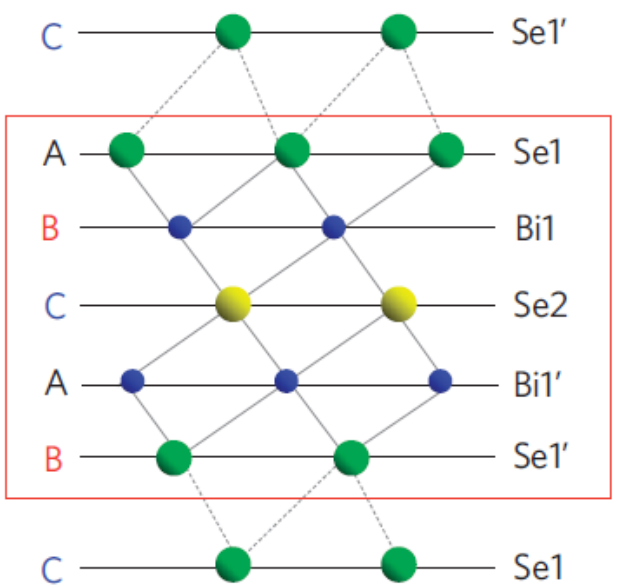

Figura 5.11: (a) Supercélula hexagonal de $\mathrm{Bi}_{2} \mathrm{Se}_{3}$ contendo 15 camadas atômicas, os vetores primitivos da célula unitária são representados por $t_{1}, t_{2}$ e $t_{3}$. (b) Visão transversal da supercélula, observa-se que há três diferentes sítios não equivalentes. (c) Figura esquemática das 5 monocamadas atômicas que formam uma QL.

Tem-se mostrado que o tamanho adequado no qual acontece a formação do cone de Dirac, ou seja, há inversão de banda devido ao SOC, corresponde a 
uma célula unitária composta de quatro Quintuple Layer's (QL), sendo uma QL o empilhamento de 5 camadas atômicas como mostrado na figura 5.11. A interação entre camadas atômicas é mais forte do que a ligação via Van der Walls entre as QL's. De modo que, a separação QL-QL é maior do que a separação entre camadas atômicas dentro das QL's, fazendo possível identificar na estrutura o limite de cada Q1 como é esquematizado na figura 5.11c.

Tendo em vista a enorme importância do SOC na descrição do sistema $\mathrm{Bi}_{2} \mathrm{Se}_{3}$, neste trabalho foi feito um estudo da evolução da estrutura de bandas em relação ao número de QL's. Foi usado o potencial de troca e correlação LDA que descreve razoavelmente a interação de Van der Walls, foi utilizado um MeshCutoff de $500 \mathrm{Ry}$, uma rede de 25 pontos K em cada direção, 2000 pontos para fazer as bandas e foi otimizado o conjunto de orbitais atômicos que formam a base, salientando que em todas as contas a estrutura foi relaxada .
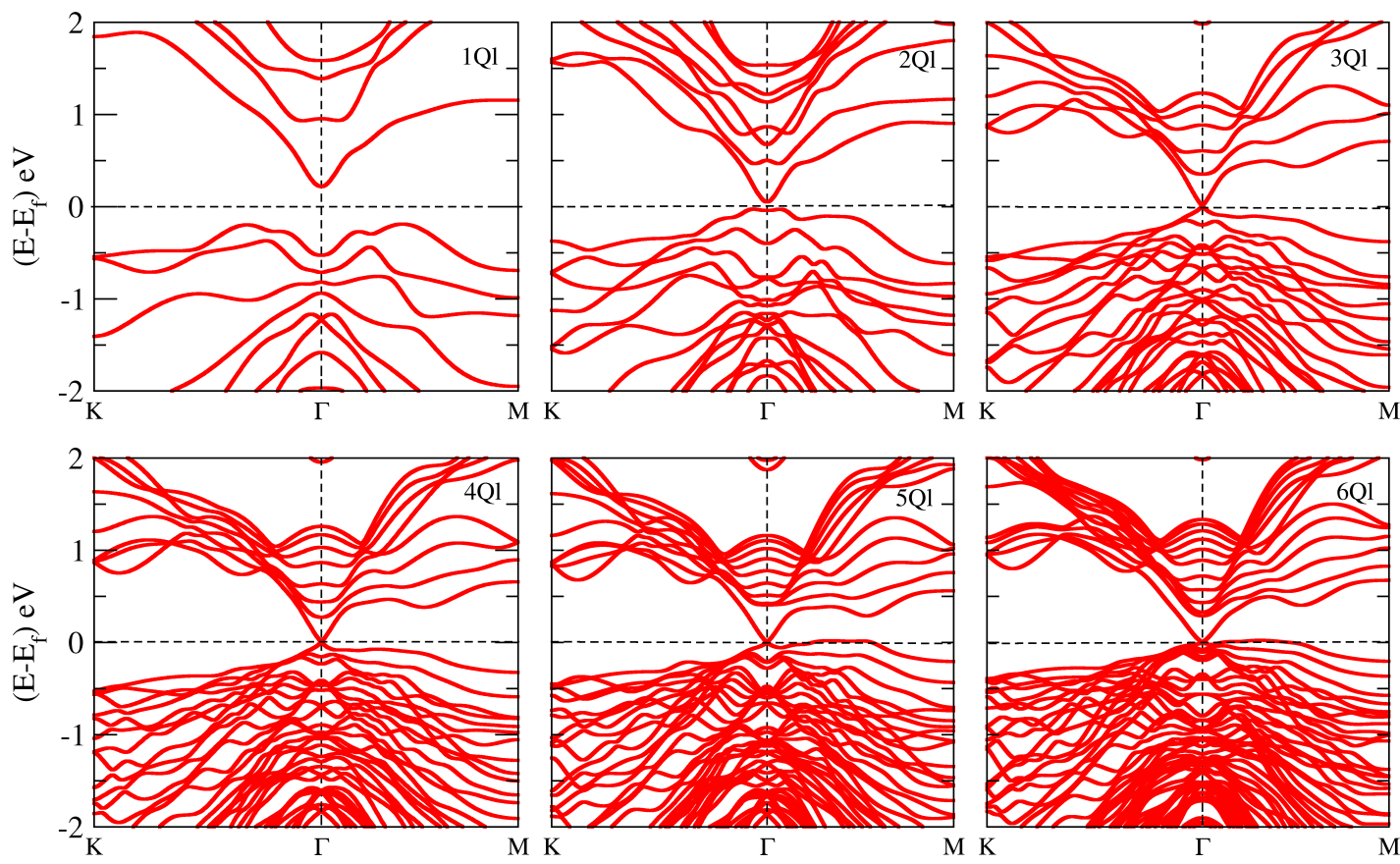

Figura 5.12: Estrutura de bandas para diferentes Ql's de $\mathrm{Bi}_{2} \mathrm{Se}_{3}$. Em este cálculo foram levados em consideração os efeitos relativísticos. A partir de 4Ql's. o SOC gera uma inversão de banda que resulta na formação de um cone de Dirac no ponto $\Gamma$.

Observa-se uma enorme correspondência entre os resultados obtidos e os 
encontrados na literatura. Verifica-se a existência do cone de Dirac a partir das quatro Ql's associado com a inversão de banda e consequentemente à formação de estados de superfície metálicos protegidos pela simetria de reversão temporal. Apesar de ter sido verificado que o $\mathrm{Bi}_{2} \mathrm{Se}_{3}$ é um TI, os mecanismos que permitem o controle dos estados de superfície nos TI com a manipulação do spin do elétron ou da simetria de reversão temporal são um tema aberto e de atual foco de estudo na matéria condensada. Dado que impurezas magnéticas adsorvidas em TI quebram a simetria de reversão temporal [52], espera-se que a adsorção de impurezas magnéticas possa ser um mecanismo de controle dos estados topológicos nos TI. Contudo, experimentalmente não é possível manipular a orientação particular do spin de cada elétron do sistema, de forma que, no sistema predomina a fase magnética de mais baixa energia. Consequentemente, embora seja de enorme interesse teórico o estudo da resposta dos TI à presença de metais de transição adsorvidos, é necessário considerar a possibilidade de ordem magnética e a configuração mais estável para uma possível aplicação prática.

\subsubsection{Ordem magnética no $\mathrm{Bi}_{2} \mathrm{Se}_{3}$ com átomos de $\mathrm{Mn}$ adsorvidos}

Hor Y. S. et al.[53], mostraram experimentalmente que o $\mathrm{Bi}_{2} \mathrm{Te}_{3}$ pode apresentar ordens magnéticas quando dopado com Mn o qual devido a seu grande momento magnético intrínseco favorece à formação de configurações magnéticas. Isto nos motivou ao estudo de fases ferromagnéticas (FM) e antiferromagnéticas (AFM) em átomos de Mn quando adsorvidos no $\mathrm{Bi}_{2} \mathrm{Se}_{3}$. Para o desenvolvimento deste trabalho, foram colocadas impurezas tanto na superfície superior quanto na inferior, e as camadas atômicas que formam as QL's foram relaxadas, entretanto foi

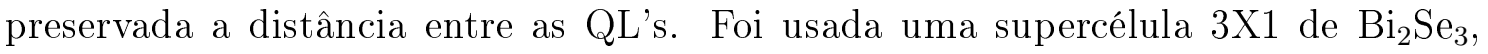
com 3 átomos de Mn em cada superfície (superior e inferior), e foram feitas as 
contas usando 4QL's e 6QL's. Foi determinado que, na superfície o sítio de adsorção mais estável do Mn é a posição B,representada na figura 5.11. Para determinar a configuração magnética mais estável foram colocada diferentes configurações iniciais no spin dos Mn, foi ligada a contribuição do SOC no cálculo autoconsistente e relaxada a parte eletrônica do sistema fixando os íons. Desta forma, foi encontrado que o sistema possui a energia mais baixa quando o momento magnético dos átomos de Mn está orientado na direção perpendicular ao plano da superfície do TI, e existe um mínimo local quando o spin dos Mn está orientado em uma direção contida no plano da superfície do TI.

Determinou-se que a configuração mais estável corresponde com a fase AFM tanto para 4Ql's quanto para 6Ql's, sendo 0,8 eV energeticamente mais favorável que a fase FM, de modo que experimentalmente espera-se que o Mn adsorvido apresente uma fase AFM, preservando os estados topológicos da superfície. A fase FM com um momento magnético total de 14.5 magnetons de Bhor quebra a simetria TR como é evidenciado na estrutura de bandas na 5.13a, de modo que o comportamento de férmions de Dirac sem massa desaparece.

Inicialmente foram usadas 4QL's, porém na configuração AFM, a interação entre impurezas de diferentes superfícies induz um momento magnético total liquido no sistema de 0.14 magnetons de Bhor orientado na direção perpendicular à superfície. Este pequeno momento magnético resulta em uma abertura de $2 \mathrm{meV}$ do gap espectral como é mostrado na figura 5.13b. Foi verificada a existência de interação entre impurezas de diferente superfície colocando spin fora do plano para os Mn adsorvidos na superfície superior e spin no plano para os Mn adsorvidos na superfície inferior, após a relaxação eletrônica o spin das duas superfícies ficou orientado na direção do mínimo global (spin fora do plano). Isto somente pode ser explicado pela interação dos Mn de superfícies diferentes. Consequentemente, considerando 6Ql's, configuração que em princípio não permite a interação entre 
impurezas de diferente superfície, a fase AFM fornece um gap nulo, de modo que, preserva-se a simetria TR. Portanto, os estados topológicos das superfícies são mantidos na estrutura de bandas.

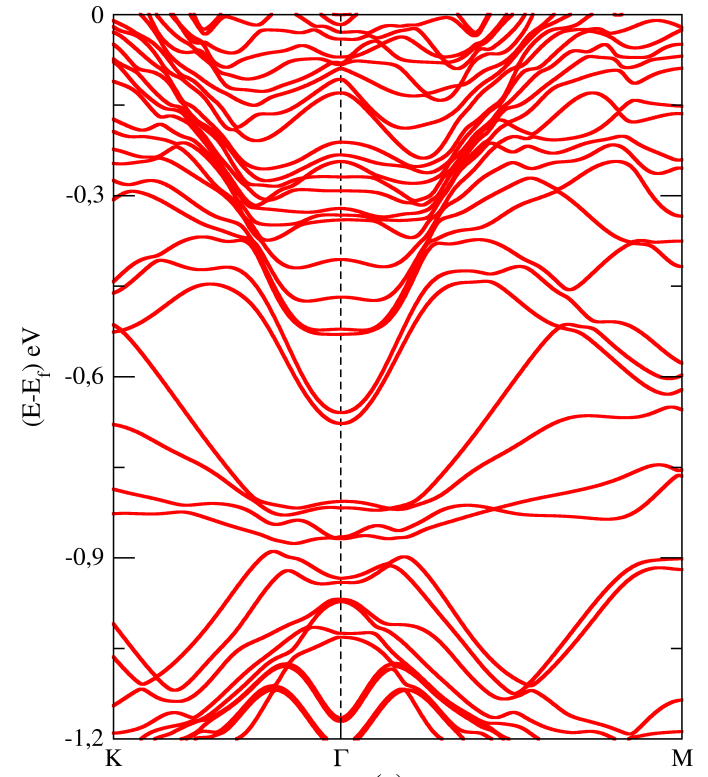

(a)

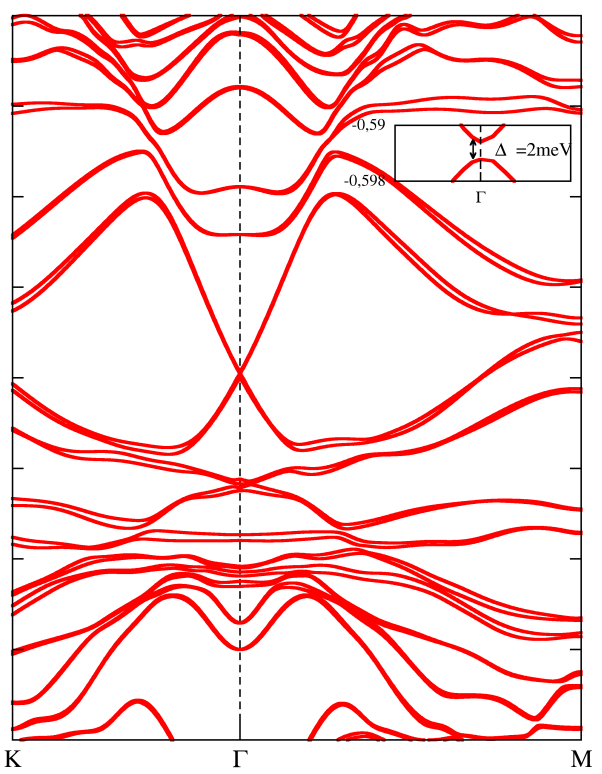

(b)

Figura 5.13: Estrutura de bandas do $\mathrm{Bi}_{2} \mathrm{Se}_{3}$ com Mn adsorvido. (a) Configuração ferromagnética. (b) Configuração antiferromagnética.

Embora o controle das propriedades eletrônicas dos TI seja de grande interesse, existem outras propostas de aplicações em spintrônica associadas com a manipulação de estados topológicos. Tendo em vista que a primeira predição teórica de estados topológicos protegidos pela simetria de reversão temporal foi baseada no estudo de um particular sistema bidimensional de átomos de carbono dispostos em forma hexagonal ${ }^{5}$, conhecido como grafeno[3], este material tem sido considerado como um dos principais candidatos para aplicações em spintrônica. Porém, o fraco SOC do grafeno não permite a observação experimental deste efeito $[3,54,55,56,57]$. Em consequência, a transmissão do SOC ao grafeno tem-se convertido em um dos objetivos dos físicos da matéria condensada. Entretanto, têm-se encontrado átomos que embora não favoreçam a formação do QSH apresentam outros fenômenos de fundamental interesse nas possíveis aplicações tal como o "Strong spin-splitting" e

\footnotetext{
${ }^{5} \mathrm{~A}$ rede com simetria hexagonal é comumente chamada de colmeia
} 
o efeito Hall quântico anômalo. Neste trabalho foram estudados os efeitos do SOC em metais de transição adsorvidos em grafeno.

\section{4 "Strong Spin-Splitting” em Grafeno}

O grafeno possui propriedades eletrônicas particulares[58,59] que são de enorme interesse para a spintrônica. Entretanto, uma meta desafiadora é controlar o spin dos elétrons através de mecanismos elétricos, por exemplo, pelo acoplamento magnetoeléctrico ou pelo SOC[60,61,62]. Isto implica que inicialmente deve-se gerar um mecanismo que aumente os efeitos do SOC no grafeno com um ambiente adequado que permita aplicações práticas na spintrônica.

Tem-se medido experimentalmente o "spin-splitting" gerado pelo SOC $\left(\Delta_{S O C}\right)$ em grafeno crescido em substratos metálicos $[63,64]$. Embora tenha sido reportado um grande $\Delta_{S O C}{ }^{6}$ [63], o resultado foi confirmado por Rader et al.[64], através de cálculos de primeiros princípios. Li et al.[65], demonstraram que o $\Delta_{S O C}$ das bandas $\pi$ do grafeno sobre $\mathrm{Ni}\left(\begin{array}{lll}1 & 1 & 1\end{array}\right)$ é de $10 \mathrm{meV}$, menor da ordem comumente usada em dispositivos. Li et al.[65], também previram que um $\Delta_{S O C}$ grande pode ser obtido no grafeno quando crescido em Au com uma certa tensão no plano grafeno. O mecanismo foi atribuído ao aumento da contribuição do momento angular orbital ao SOC devido à presença de átomos pesados.

Um grande número de formas têm sido propostas para sintonizar as propriedades eletrônicas e magnéticas do grafeno. Recentemente, vários grupos teóricos e experimentais têm relatado que a adsorção de metais ou de pequenas nanopartículas inorgânicas (incluindo $\mathrm{Au}, \mathrm{Ag}$ e $\mathrm{ZnO}$, etc) podem provocar variações significativas nas propriedades do grafeno[66]. No entanto, nesses trabalhos não foram estudados os efeitos do SOC no grafeno. Motivados pelas evidências

\footnotetext{
${ }^{6} 1000$ vezes maior do que o gap gerado pelo SOC no grafeno pristino.
} 
experimentais e pelos fortes efeitos do SOC na estrutura eletrônica do $\mathrm{Au}$ e Pt, como estudado por Glantsching e Ambrosch[67], neste trabalho exploraram-se se estes átomos pesados adsorvidos em grafeno podiam melhorar o SOC dos sistemas ou gerar efeitos que possam ser usados em possíveis aplicações de spintrônica.

O presente trabalho, concentrou-se nos efeitos do SOC em bandas $\pi$ do grafeno com átomos adsorvidos de $\mathrm{Au}$ e $\mathrm{Pt}$ (abreviado como $\mathrm{Au} / \mathrm{Gr}$ e $\mathrm{Pt} / \mathrm{Gr}$ ) a partir de um método de primeiros princípios usando o código SIESTA-SOC. Três tipos de configurações de adsorção no sistema pristino foram estudadas: adsorvido acima de um átomo de Carbono (A), no meio de um hexagono formado por carbonos (B) e acima da ligação C-C (C) como observa-se na figura 5.14. Para entender o efeito da adsorção do Au e Pt como impurezas no grafeno foram consideradas supercélulas de grafeno do tamanho $4 \times 4$ e 6x6 com um único metal adsorvido, de forma que, a distâncias entre impurezas são maiores do que 9,5 Ang e, portanto não existe interação direta entre os átomos adsorvidos.

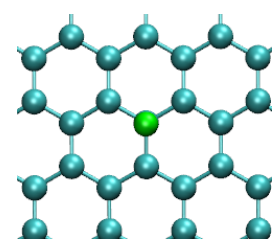

(a)

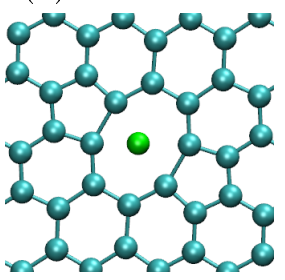

(d)

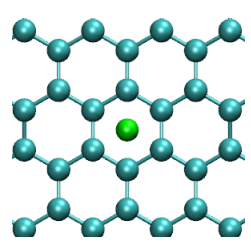

(b)

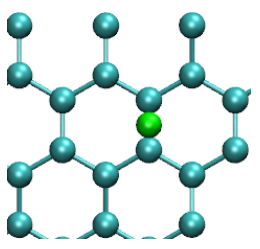

(c)

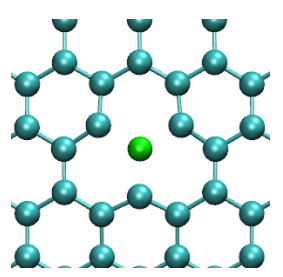

(e)

Figura 5.14: Figura esquemática dos sítios de adsorção no grafeno estudados. (a) Acima do átomo de carbono. (b) No meio no hexágono. (c) No meio da ligação carbono-carbono. (d) No defeito 585. (e) Em uma mono vacância.

Experimentalmente, se a energia de ligação não é muito alta os átomos têm uma liberdade para se movimentar no gás de elétrons bidimensional dos elétrons $\pi$ 
do grafeno. Contudo, se a impureza é adsorvida em um defeito estrutural da rede, tal como uma vacância, o átomo adsorvido fica preso neste mínimo de potencial. Neste trabalho, estudaram-se os efeitos do SOC nos metais de transição Au e Pt quando adsorvidos em vacâncias em grafeno como esquematizado nas figuras 5.14d e 5.14e. Encontrou-se que a posição B é a mais estável para o $\mathrm{Au}$, foi verificado o grande valor do desdobramento de algumas bandas nesta posição o qual foi denominado "strong spin-splitting". Embora este fenômeno não acontece no nível de Fermi evidencia um possível caminho para a transferência de SOC ao grafeno. Nas outras posições de adsorção não existem efeitos relevantes do SOC, mesmo quando o Au é adsorvido em vacâncias.

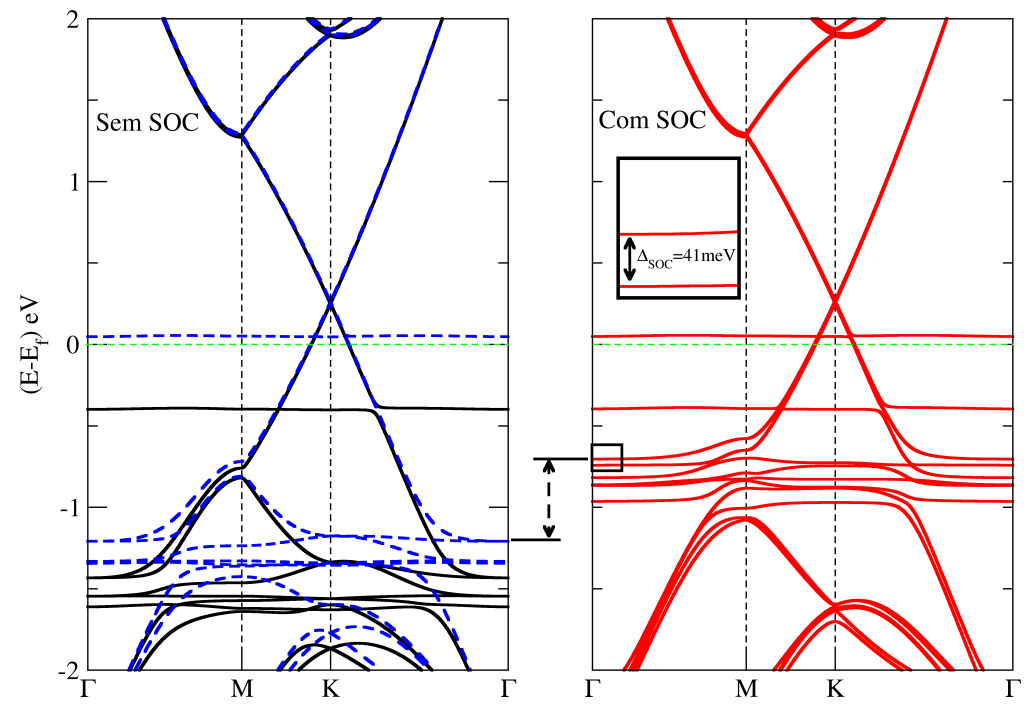

Figura 5.15: Au adsorvido na posição B em uma supercélula 4X4 de grafeno. A estrutura de bandas sem levar em consideração o SOC apresenta estados degenerados no ponto $\Gamma$. Esta degenerescência é levantada quando se considera o SOC. Adicionalmente, estes estados são deslocados em até $0.5 \mathrm{eV}$. O requadro representa uma ampliação destes níveis deslocados, evidenciando uma separação de $41 \mathrm{meV}$ entre eles.

O átomo de $\mathrm{Pt}$, quando adsorvido no grafeno, fornece uma imagen da forma como o SOC perturba a estrutura de bandas. Na posição B a qual corresponde à posição de adsorção mais estável, verrificamos quebras de degenerescência da ordem de $0.2 \mathrm{eV}$. Entretanto, estes efeitos aparecem relativamente longe do nível de Fermi, da mesma forma que no Au. Quando o átomo de Pt é adsorvido em 
uma monovacância de uma supercélula 6X6 de grafeno ele causa um efeito enorme interesse na procura do aumento do SOC no grafeno. Nesta posição o SOC abre um gap da ordem de $20 \mathrm{meV}$ como é evidenciado na figura 5.16. Não existe registro ou indicios de que este fenômeno tenha sido observado ou discutido na literatura. Nas outras posições o SOC não gera efeitos relevantes.
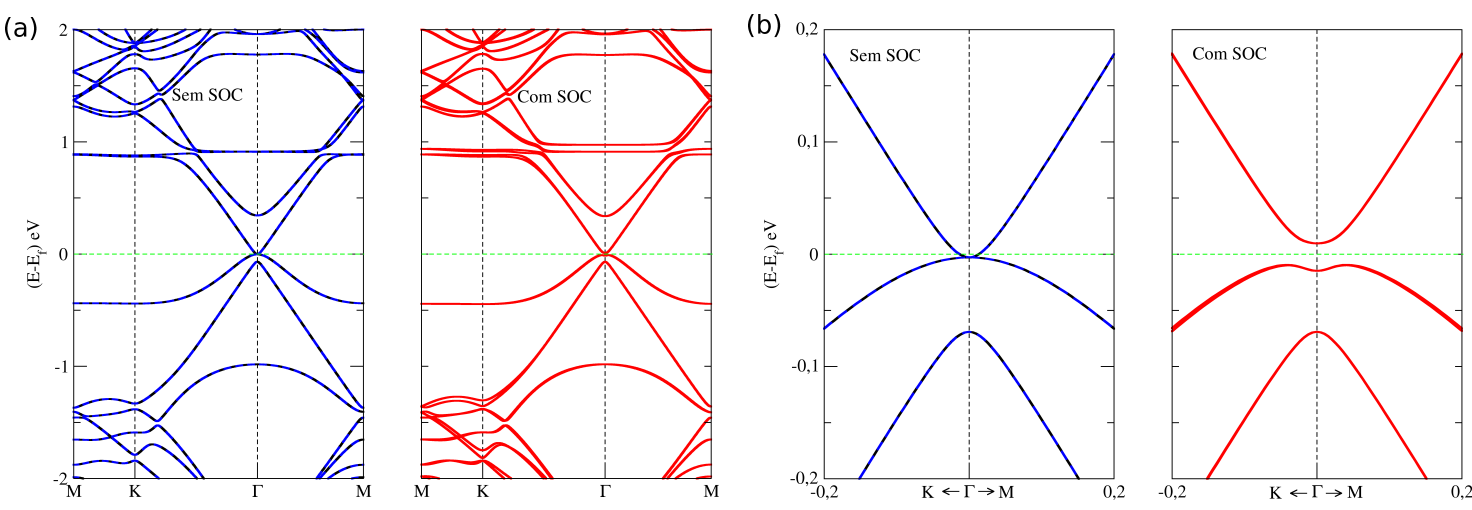

Figura 5.16: Estrutura de bandas do Pt quando adsorvido na mono-vacância de uma supercélula 6X6 de grafeno. (a) Estrutura de bandas com e sem levar em cosideração o SOC. A cor preta e azul representam as componente do spin up e down, respetivamente. Enquato que a cor vermelha está associada com a estrutura de bandas levando em consideração o SOC. (b) Zoom nas proximidades no nível de Fermi, evidenciando as diferenças na estrutura de bandas com e sem SOC. 


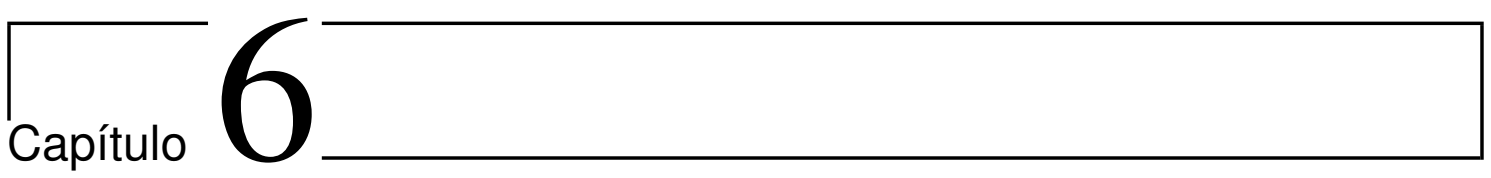

\section{Fases topológicas em uma rede de}

\section{"baricentros" com simetria hexagonal}

A dificuldade em controlar as propriedades dos férmions de Dirac sem massa no grafeno motivou a procura de materiais que apresentem características similares ao grafeno, mas que permitam o controle de suas propriedades. Esta procura tem fornecido bons resultados. Foi mostrado que os sistemas com simetria hexagonal tais como Siliceno, Germaneno e "Graphynes"[7,10], além de apresentar as caraterísticas eletrônicas do grafeno, permitem a existência de novos fenômenos de enorme interesse teórico e experimental. Após inumerosos estudos destes sistemas, baseados na "engenharia de superfície" Kenjiro K. et al.[68], apresentaram uma nova proposta conhecida como grafeno molecular.

A adsorção de átomos e/ou moléculas em superfícies de materiais é um procedimento corriqueiro nas atividades de pesquisa em materiais. Entretanto, quando esta adsorção de átomos/moléculas são controladas e monitoradas espacialmente é possível construir estruturas cristalinas artificiais com propriedades físicas singulares. O grafeno molecular é um interessante exemplo desta "engenharia de superfície". A partir da formação de uma rede triangular de moléculas de CO depositadas, 
via STM, em uma superfície de $\mathrm{Cu}(111)$, Kenjiro K. et al.[68], encontraram altas concentrações de elétrons (picos na densidade eletrônica) localizadas nos baricentros associados aos triângulos formados pelas moléculas de $\mathrm{CO}{ }^{1}$. A deposição de moléculas de $\mathrm{CO}$ em uma distribuição espacial com simetria triangular resulta na disposição hexagonal dos baricentros, de forma que estes formam uma rede de colmeia, como mostrado na figura 6.1a. Esta rede hexagonal de baricentros apresenta uma densidade de estados característica de férmions de Dirac sem massa (ver figura 6.1b), com uma estrutura de bandas próxima ao nível de Fermi em formato cônico como é ilustrado na figura 6.1c. Por estas singulares características, este sistema foi batizado com o nome de grafeno molecular. Embora, o SOC do grafeno molecular seja maior que o do grafeno pristino o qual possibilita sua utilização na spintrônica, não existe um método de fabricação que permita a aplicação prática e eficiente deste material. Porém, continua a atrair interesse pela origem física de suas propriedades. A rede hexagonal de baricentros da mesma forma que o grafeno pode ser estudada considerando duas subredes A e B ${ }^{2}$.
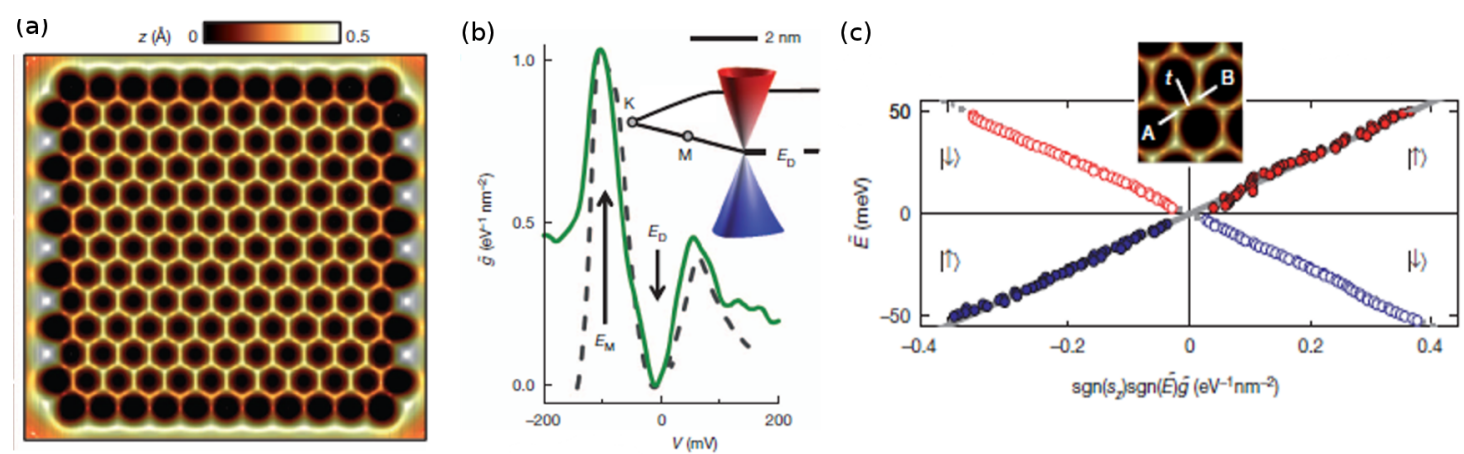

Figura 6.1: Construção da rede de baricentros pela deposição de moléculas de CO, via STM, na superfície de $\mathrm{Cu}(111)$. (a) Imagens de STM da rede hexagonal de baricentros. As regiões obscuras correspondem às moléculas e as regiões brilhantes aos baricentros se comunicando via uma rede hexagonal. (b) Densidade de estados da rede hexagonal de baricentros. (c) Estrutura de bandas no nível de Fermi, a cor vermelha corresponde aos estados desocupados e a cor azul aos estados ocupados, o spin $\downarrow$ é representado pelas bolas cheia e o spin $\uparrow$ pelas bolas vazias.

\footnotetext{
${ }^{1}$ A palavra "baricentro" refere-se ao centro de uma figura geométrica.

${ }^{2} \mathrm{O}$ spin $\downarrow$ (spin down) e o spin $\uparrow$ (spin up), são definidos em termos da projeção do spin no eixo $z$. No texto, será considerado o eixo $z$ como o eixo preferencial e o spin $\downarrow$ será representado pela cor azul e o spin $\uparrow$ pela cor preta.
} 
Embora não tenha sido mostrado explicitamente que a rede hexagonal de baricentros possui estados QSH, foram observadas fases topológicas não triviais. Deste modo, o grafeno molecular traz uma informação relevante: é possível gerar estados topológicos não triviais quando se constrói uma rede hexagonal de baricentros a partir da deposição de moléculas de $\mathrm{CO}$ em um gás de elétrons bidimensional contido numa superfície metálica.

A relativa facilidade de se obter folhas de grafeno, a engenharia de estados robustos QSH em grafeno através da adsorção de átomos de Indio e Tálio proposta por Weeks et al.[69] e a engenharia de estados QHA em grafeno via adsorção de metais de transição desenvolvida por Ding et al.[70], nos motivou a busca de um sistema de baricentros construído a partir da montagem de átomos sobre o "gás de elétrons bidimensional" formado pelos elétrons $\pi$ do grafeno. Espera-se que uma rede triangular de átomos adsorvidos no grafeno gere um sistema de baricentros com simetria hexagonal e forte SOC, de forma que, uma fase topológica tal como o QHA ou QSH apresente estados metálicos estáveis na borda mais robustos do que no grafeno pristino.

Nas seções seguintes será mostrado que é possível a existência de uma rede hexagonal de baricentros formada pela adsorção de átomos no grafeno, de forma que este sistema apresente fases topológicas não triviais. Embora a palavra "baricentro" refere-se ao centro de uma figura geométrica, será usada de forma indistinta para fazer referência aos picos de densidade de estados localizados no centro geométrico das moléculas adsorvidas particularmente numa distribuição triangular ou o centro geométrico destas. 


\subsection{Rede hexagonal de baricentros}

Os efeitos do SOC no grafeno têm sido amplamente estudados $[55,56,57,71,72]$, inclusive existem propostas teóricas para aumentar estes efeitos de forma que sejam observáveis experimentalmente[3,39,75]. Os átomos pesados são de grande interesse para estes fins, devido a seu intrínseco e forte SOC[65,74]. De acordo com Weeks et al.[69], (2010) e Ding et al.[70], átomos pesados adsorvidos em grafeno estabilizam fases topológicas mais robustas do que as presentes no grafeno pristino. Assim, a física entorno do ponto de Dirac deve ser modificada, principalmente, devido à indução do forte SOC intrínseco dos átomos adsorvidos. Consequentemente, a procura de um sistema de baricentros formado a partir de grafeno que de forma efetiva apresente um forte SOC enfoca-se na adsorção de átomos pesados.

De acordo como o modelo de Kane e Mele[3], átomos adsorvidos no grafeno que favoreçam a posição B (ver figura 5.14b) podem mediar eficazmente a dependência do "hopping" dos segundos vizinhos com o spin, e simultaneamente evitar maiores efeitos, tais como a quebra local da simetria entre as subredes, como no caso da adsorção na posição C (ver figura 5.14c). Portanto, embora existam efeitos da interação spin-órbita quando átomos são adsorvidos em outras posições, a procura de um sistema de baricentros foi enfocada em átomos cuja posição mais estável quando adsorvidos no grafeno corresponde à posição B.

Após uma intensa procura encontramos que o Rutênio (Ru) quando adsorvido no grafeno $(\mathrm{Ru} / \mathrm{Gr})$ hibridiza de forma que, para algumas concentrações em particular, permite a existência de uma rede hexagonal de baricentros. A estrutura de bandas deste metal apresenta uma forte perturbação gerada pelo SOC como é mostrado na figura 6.2. Dado que a posição mais estável na qual o $R u$ é adsorvido corresponde com a posição $\mathrm{B}$, em principio, este átomo permite a existência de fases topológicas não triviais. 

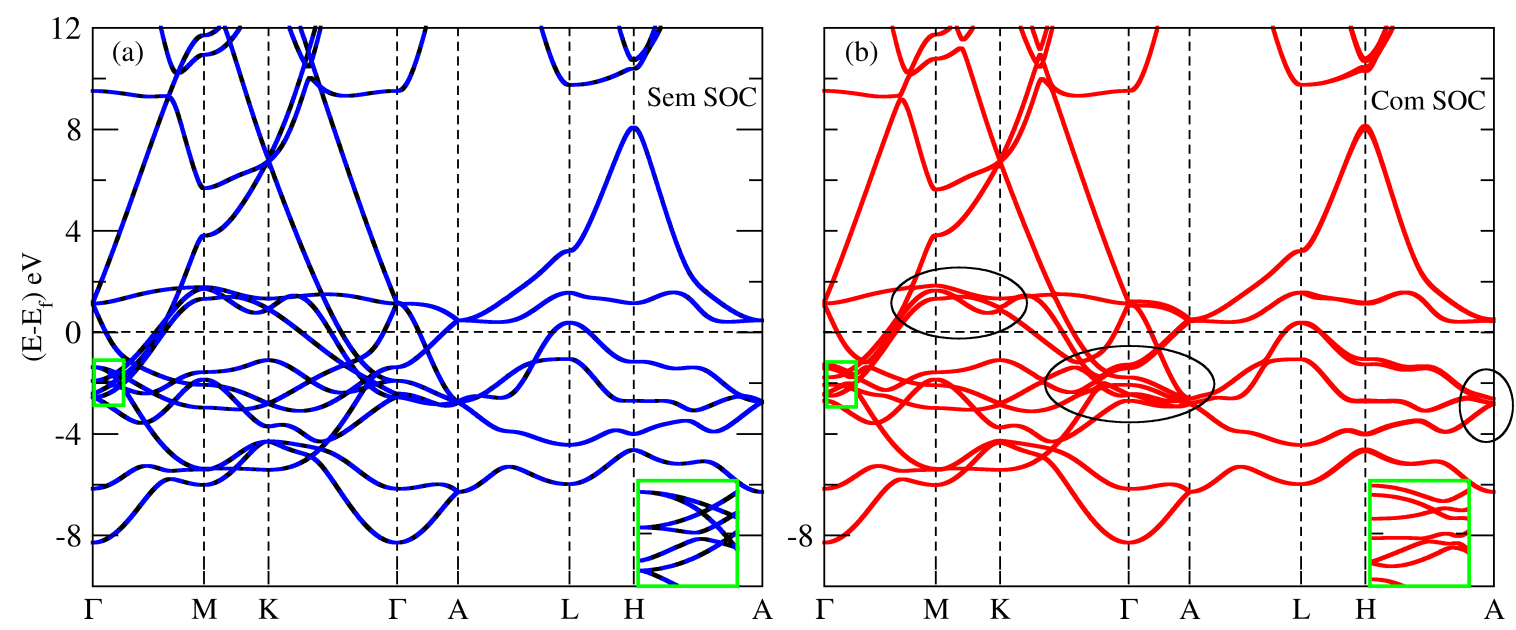

Figura 6.2: (a) Estrutura de bandas do "bulk"de Rutênio considerando polarização de spin. O quadrado verde na parte inferior direita corresponde com um zoom na estrutura de bandas no ponto $\Gamma$ embaixo do nível de Fermi. (b) Usando o código SIESTA-SOC foi feita a estrutura de banda do "bulk"de Rutênio levando em consideração a interação spin-órbita. Observa-se uma mudança dos níveis no quadro do zoom e um spin-splitting de até $0.1 \mathrm{eV}$. Nas elipses são indicadas as regiões onde os efeitos do SOC são mais evidentes.

Levando em consideração que no átomo de $\mathrm{Ru}$ os orbitais de valência são descritos pela configuração orbital $4 d^{7} 5 s^{1}$, os orbitais atômicos que prioritariamente hibridizam com os orbitais $s p^{2} p_{z}$ do grafeno possuem caráter $d$ e $s$. A simetria local do átomo de $\mathrm{Ru}$ quando adsorvido no grafeno corresponde ao grupo pontual de simetria $C_{6 v}$. A decomposição dos orbitais $d$ nas representações do grupo de simetria $C_{6 v}$ permite inferir que os orbitais responsáveis pela adsorção do átomo de Ru são os $d_{x^{2}-y^{2}}, d_{x z}, d_{y z}$ e $d_{y x}$, pois hibridizam com os orbitais $\pi$ do grafeno. A análise da simetria mostra que o orbital $d_{z^{2}}$ contribui minoritariamente na hibridização do átomo de Ru quando adsorvido no sítio B. Se não é levada em consideração a polarização de spin, na densidade de estados aparece um pico embaixo do nível de Fermi associado com uma banda de energia de estados localizados ocupados cuja contribuição majoritária vem do orbital $d_{z^{2}}$ do Ru. Analogamente, se este metal de transições tende a ficar na configuração eletrônica efetiva $d^{8}$, observa-se acima do nível de Fermi um pico com contribuição majoritária do orbital $5 s$. Esta análise verifica-se na densidade de estados projetada (PDOS) nos orbitais do átomo do Ru quando comparada com a densidade de estados (DOS), como é apresentado nas 
figuras $6.3 \mathrm{~d}$ e $6.3 \mathrm{~b}$.

Quando a polarização de spin é considerada na PDOS dos orbitais atômicos do Ru, observa-se um forte efeito do potencial de troca que resulta na separação das componentes "up" e "down" dos estados localizados $d_{z^{2}}$ e $5 s$ como é evidenciado na estrutura de bandas da figura 6.3e e na PDOS da figura figura 6.3f.
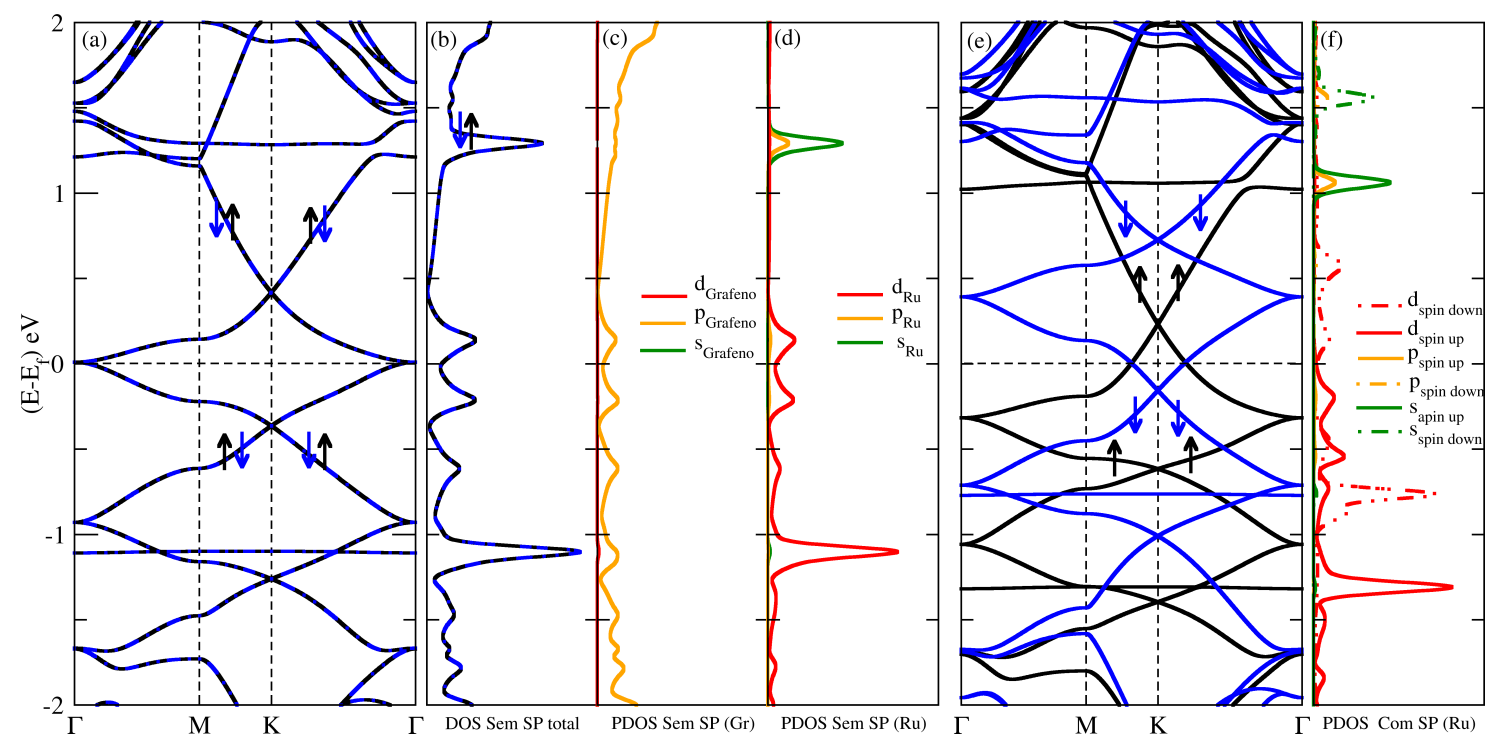

Figura 6.3: Estrura de bandas do sistema formado pela adsorção do $\mathrm{Ru}$ em uma supercélula 5X5 de grafeno. A cor azul e preta representam estados com spin "down" e "Up", respectivamente. Quando não é levada em consideração a polarização de spin temse: (a)Estrutura de bandas. (b) Densidade total de estados. (c) Densidade de estados projetada nos orbitais do grafeno. (d) Densidade de estados projetada nos orbitais do Ru. Quando se considera a polarização de spin tem-se: (e) Estrutura de bandas. (f) Densidade de estados projetada nos orbitais do átomo de $\mathrm{Ru}$.

Além disso, pode-se ver claramente a presença de uma contribuição relevante dos orbitais $d_{x^{2}-y^{2}}, d_{x z}, d_{y z}$ e $d_{y x}$ do $\mathrm{Ru}$ na formação da estrutura de bandas entorno do nível de Fermi, de modo que, a interação Ru-grafeno resulta em uma estrutura de bandas com dois cruzamentos no ponto K (figura 6.3a) ou quatro quando se introduz um campo de "exchange" (figura 6.3e), ao invés do único cruzamento bidegenerado presente no ponto $\mathrm{K}$ da estrutura de bandas do grafeno pristino. A DOS sem levar em consideração a polarização de spin fornece um formato característico de um cone de Dirac nas proximidades do nível de energia no qual aparecem os cruzamentos das bandas no ponto K (figura 6.3b). A contribuição à 
energia dos estados do grafeno e do Ru são nulas nesses valores de energia, o qual permite atribuir o nome de "Cone de Dirac" a estes cruzamentos.

Quando estuda-se a contribuição aos cones de Dirac dos estados do grafeno (figura 6.3c) em relação aos estados do Ru (figura 6.3d), percebe-se que o intervalo de energia no qual se formam os cones corresponde a uma região de "ressonância" entre os estados do grafeno e os estados do metal. Os cones de Dirac são formados por contribuições similares dos estados do Ru e do grafeno, de forma que, a estrutura de bandas do $\mathrm{Ru}$ quando adsorvido no grafeno ao invés de estar associada unicamente aos estados do grafeno, está relacionada com estados de um "novo sistema" resultado da hibridização Ru-Grafeno. Será mostrado que este sistema corresponde com uma rede hexagonal de baricentros.
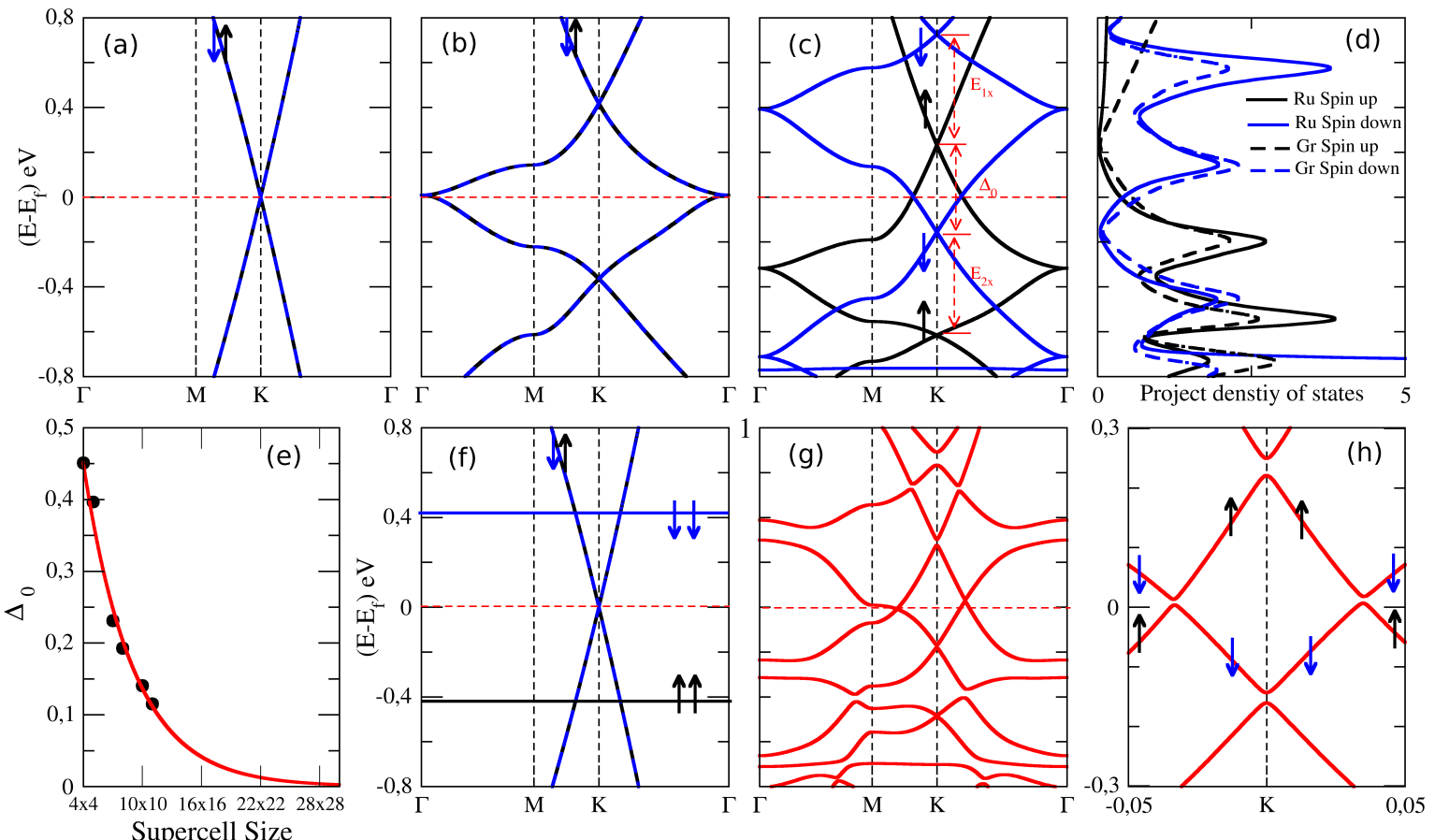

Figura 6.4: Sequência da variação da estrutura de bandas. (a) Estrutura de bandas do grafeno pristino. Estrutura de banda do Ru quando adsorvido no grafeno, (b) sem considerar a polarização de spin e (c) levando em consideração a polarização de spin. (d) Densidade de estados projetada nos orbitais do grafeno e nos orbitais átomicos do Ru. (e) Variação da separação dos cones mais proximos ao nível de Fermi em relação à variação do tamanho da supercélula. (f) Figura pictórica da estrutura de bandas para uma supecélula muito grande. (g) Estrutura de bandas levando em consideração o SOC. (h) Configuração dos spin nas proximidades do nível de Fermi. 
A PDOS levando em consideração a SP (figura 6.4d) permite ver a "ressonância" das contribuições do Ru e dos carbonos à densidade eletrônica total. A energia de troca somente gera uma quebra da degenerescência de spin (figura 6.4c), ou seja, o caráter de férmions de Dirac sem massa dos cones presentes na estrutura de bandas não dependem desta contribuição. Consequentemente, a presença dos cones de Dirac deve-se unicamente ao "campo cristalino" gerado pela presença da distribuição triangular dos Ru adsorvidos no grafeno. Se este campo cristalino está associado à rede hexagonal de baricentros, espera-se que o comportamento particular deste sistema não dependa da interação direta entre os Ru. Mesmo a baixas concentrações de $\mathrm{Ru}$ mantendo a simetria triangular o sistema deve apresentar quatro cones de Dirac no ponto K. Isto foi verificado mediante a variação do tamanho da célula de grafeno na qual situa-se o Ru. Mesmo considerando concentração de Rutênio de $0.82 \%$ (supercélula $11 \mathrm{X} 11,1 \mathrm{Ru}$ por cada 121 carbonos), o sistema apresenta quatro cones de Dirac. O fato de que o comportamento é mantido para distâncias $\mathrm{Ru}-\mathrm{Ru}$ maiores do que 26Ang é um argumento claro para afirmar que os $\mathrm{Ru}$ interagem indiretamente. O mecanismo pelo qual é gerada esta interação associa-se com uma forte hibridização dos estados do Ru com os estados do grafeno. Assim, diz-se que os Ru comunicam-se via grafeno. A diferença de energia dos cones nas proximidades do nível de Fermi, $\Delta_{0}$, diminui proporcionalmente à concentração de $\mathrm{Ru}$ (figura 6.4e), de modo que conforme aumenta a célula unitária a interação indireta dos Ru decresce e a contribuição deste átomos à formação dos cones diminui. Como é evidenciado na figura (figura 6.4e) no limite de distância Ru-Ru muito grandes o sistema "recupera" o comportamento do grafeno com a presença de estados localizados do Ru (figura 6.4f).

A forma como as interações: hibridização com Ru, potencial de "exchange" e spin-órbita, modificam a estrutura de banda do grafeno grafeno pristino pode ser descrita da seguinte forma: 
- O grafeno pristino apresenta um único cone de Dirac degenerado em spin no ponto K e K'.

- O Rutênio quando adsorvido no meio do hexágono de carbonos do grafeno gera um campo cristalino que no caso particular das famílias 4X4 e 5X5 gera dois cones de Dirac com degenerescência de spin no ponto K e K'.

- Quando é levado em consideração o potencial de "exchange", quebra-se a degenerescência de spin de modo que a estrutura de bandas apresenta quatro cones no ponto K e K'.

- Quando o tamanho da célula unitária tende ao infinito o sistema recupera a memória do grafeno, os estados do Ru não contribuem à formação dos cones entorno do nível de fermi e os dois cones mais afastados do nível de fermi ficam localizados.

- O SOC quebra a degenerescência nos cones (figura 6.4g), de forma que o sistema inicialmente metálico pode ser considerado como um semicondutor com uma singular configuração do spin nas proximidades do nível de Fermi (figura 6.4h).

O estudo da variação da concentração de Ru leva à análise de particularidades na formação da rede hexagonal de baricentros para as diferentes supercélulas. No entanto, para a apresentação desta análise precisa-se da classificação das diferentes concentrações em "famílias de supercélula" que apresentam comportamentos muito similares. Os tamanhos das supercélulas usadas foram divididas em tres grupos: $3 n X 3 n,(3 n+1) X(3 n+1)$ e $(3 n+2) X(3 n+2)$, representadas pelas supercélulas do tamanho 3X3, 4X4 e 5X5, respectivamente. Estes tamanhos de supercélula além de diferenciar-se na posição no espaço recíproco na qual se formam os cones em relação à zona de Brillouin de uma célula unitária do grafeno (figura 6.5), apresentam uma diferença na origem física deste comportamento. 
(a)

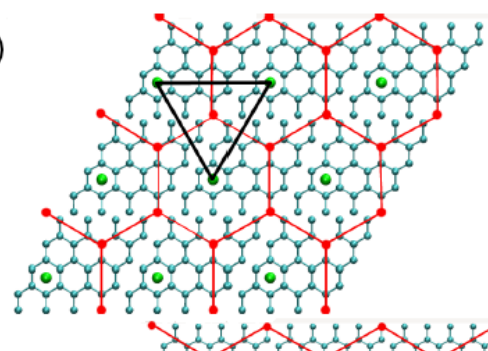

(C)

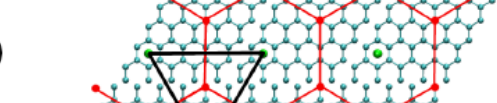

e)

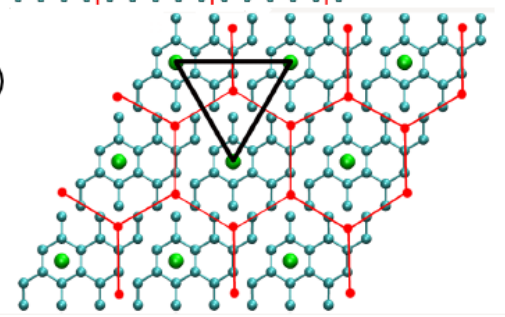

(b)

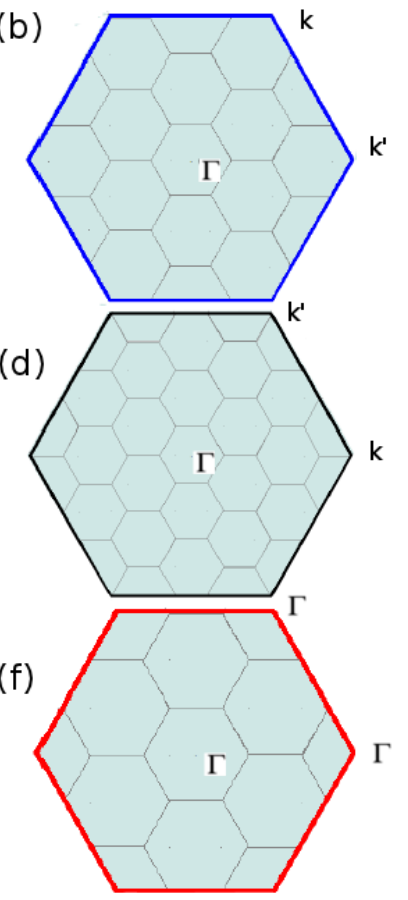

Figura 6.5: $\mathrm{O}$ posição dos baricentros em relação aos triângulos de $\mathrm{Ru}$ são esquematizados nas figuras (a), (c) e (e) para as supercélulas de tamanhos 4X4, $5 \mathrm{X} 5$ e 3X3, respectivamente. As linhas vermelhas evidenciam a simetria hexagonal dos baricentros. A zona de Brillouin das supercéclulas 3X3, 4X4 e 5X5 e a localização dos pontos de alta simetria em relação a uma célula unitária são apresentados nas figuras (f), (b) e (d), respectivamente.

A formação de uma rede hexagonal de baricentros, em principio, não depende da interação direta entre Ru. Portanto, a configuração 2X2 foi excluída do estudo já que este tamanho de supercélula permite esta interação. Foi encontrado que esta configuração induz fortes perturbações no grafeno fazendo com que o comportamento de férmions de Dirac seja modificado. Adicionalmente, não permite a formação de um sistema de baricentros, de modo que há uma diferença fundamental com a classificação das famílias consideradas dado que nestas não há efeitos na estrutura de bandas associada com a interação direta dos Ru.

As estruturas de bandas da família 3X3 não apresentam cones de Dirac. Adicionalmente, a LDOS desta família não apresenta picos na densidade de estados associados com uma rede hexagonal de baricentros. A título de exemplo é ilustrado na figura 6.6 a estrutura de bandas e LDOS para uma supercélula 6 X6 de grafeno. 

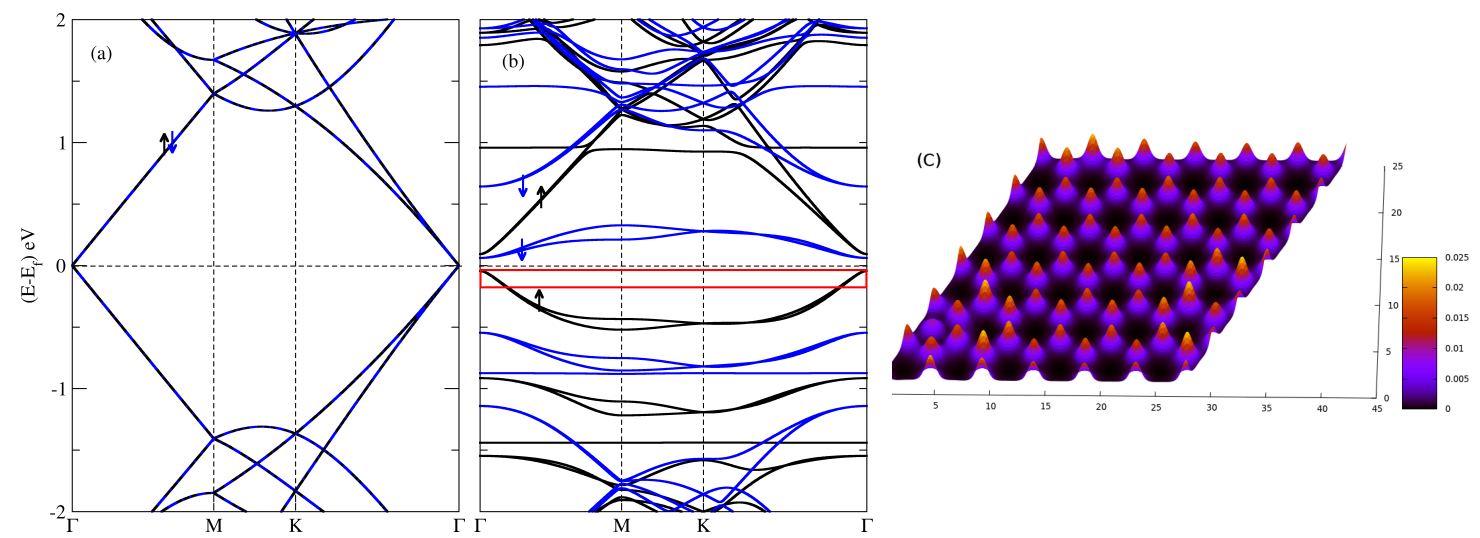

Figura 6.6: (a) Estrutura de bandas da supercélula 6X6 de grafeno. (b) Estrutura de banda do Ru quando depositado no grafeno na posição B. O comportamento cônico no ponto $\Gamma$ desaparece, a diferença da estrutura de banda do grafeno pristino. O retângulo vermelho representa a região de integração da LDOS apresentada em (c). Esta região de integração corresponde a um intervalo de energia de $20 \mathrm{meV}$ embaixo do nível de Fermi.

A LDOS na região de integração representada em vermelho na figura $6.6 \mathrm{~b}$ apresenta o mesmo formato para todas as supercélulas da família 3X3. Este particular comportamento das supercélulas com tamanhos múltiplos de três está relacionado com o sítio no qual situa-se o centro geométrico dos triângulos da rede de Ru. Como é representado pictoricamente na figura 6.5. Nas famílias $4 \mathrm{X} 4$ e 5X5, o centro de um triângulo de $\mathrm{Ru}$ (baricentro) situa-se em um átomo de carbono, enquanto que na família 3X3 o centro do triângulo de Ru está no meio de um hexágono de carbonos. A formação dos baricentros esta relacionada com o tamanho da geometria, para entender esta interessante relação é necessário compreender a origem física da rede hexagonal de baricentros.

Nas LDOS das famílias 5X5 e 4X4, mostradas nas figuras 6.7 e figura 6.8 respectivamente, nota-se a presença de um pico na densidade de estados localizado onde se situam os baricentros. Os cones de Dirac acima do nível de Fermi na família 4X4 associam-se com a presença de picos na LDOS nos baricentros. No entanto, este pico no baricentro vem acompanhado de picos na LDOS nos primeiros vizinhos do Ru. Denominou-se esta disposição de estados localizados de potencial de baricentros. Os picos da LDOS nos primeiros vizinhos dos baricentros foram 

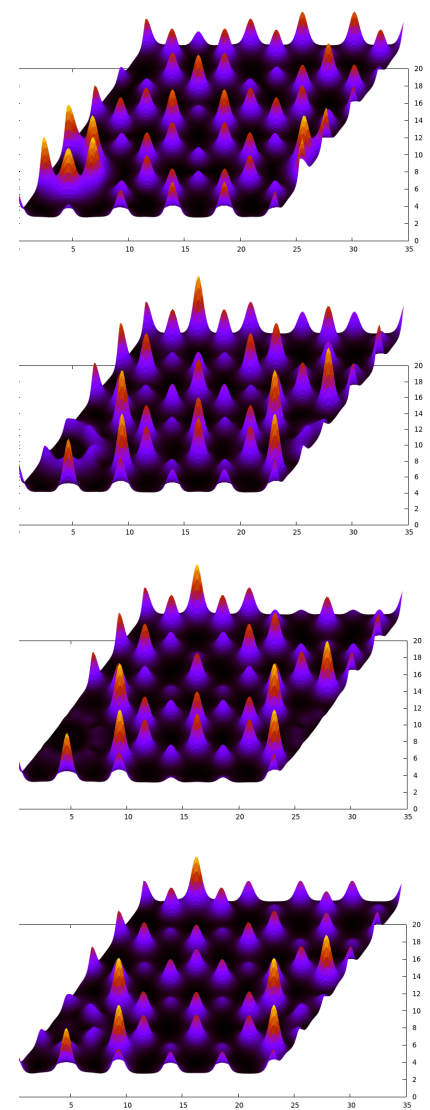

Spin Down
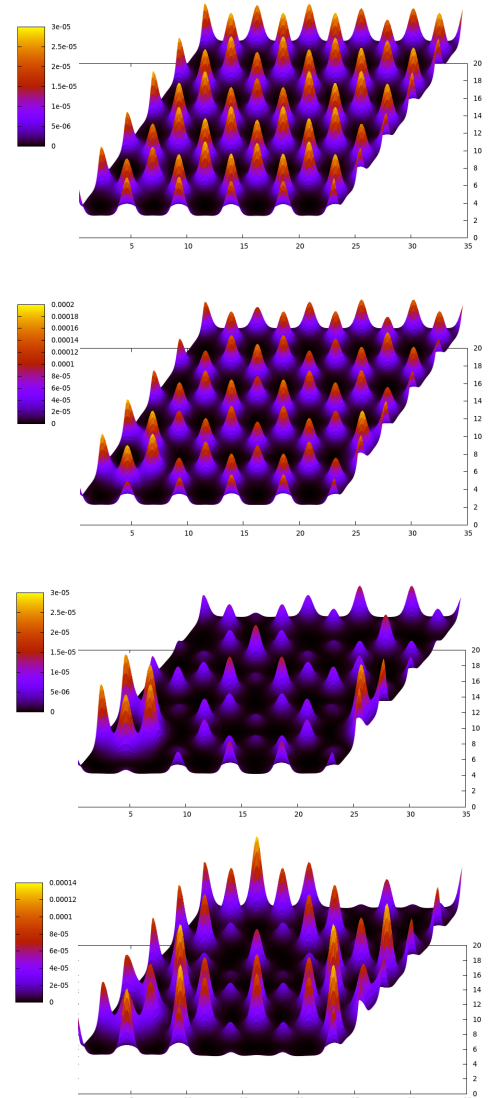

Spin Up
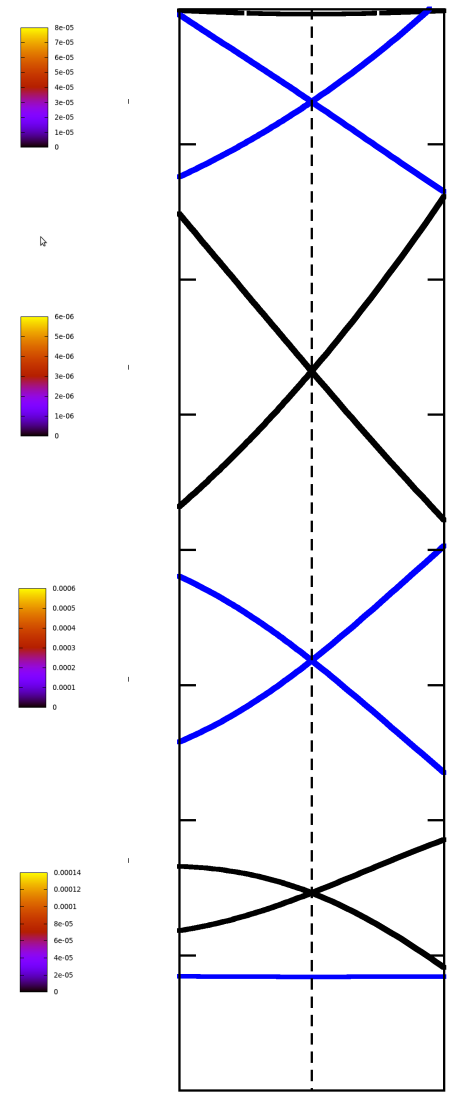

$\mathrm{M}<\mathrm{K} \rightarrow \Gamma$

0,2 0,2

Figura 6.7: LDOS por spin para cada cone apresentado na parte direita da figura. Este cones estão associados com a estrutura de bandas da supercélula 5X5. A região de integração é de $20 \mathrm{meV}$ em torno de cada cone. É importante salientar que, embora na região de integração das LDOS o cone é formado por bandas com uma única componente de spin nas outras direções exitem bandas com o spin oposto (não mostradas na figura). Desta forma, os estados localizados que contribuem majoritariamente à formação dos "cones $\downarrow$ " (cones down - cor azul) estão representados nas LDOS correspondentes ao spin $\downarrow$ (spin down). Analogamente ocorre para os cones $\uparrow$ (cones up - cor preta).

denominados antibaricentros. Estes ocorrem junto com picos de estados localizados nos segundos vizinhos do $\mathrm{Ru}$, e formam os cones desocupados da estrutura de bandas da família 4X4. Em contraste, na família 5X5 os baricentros acompanhados de picos na LDOS nos segundos vizinhos dos Ru contribuem à formação dos cones ocupados. Entretanto, os cones ocupados associam-se com os antibaricentros e possuem picos da LDOS nos primeiros vizinhos do Ru. Esta informação foi verificada calculando os coeficientes da função de onda no ponto K. Portanto, embora as contribuições dos baricentros com distribuição hexagonal sejam mais favoráveis energeticamente 

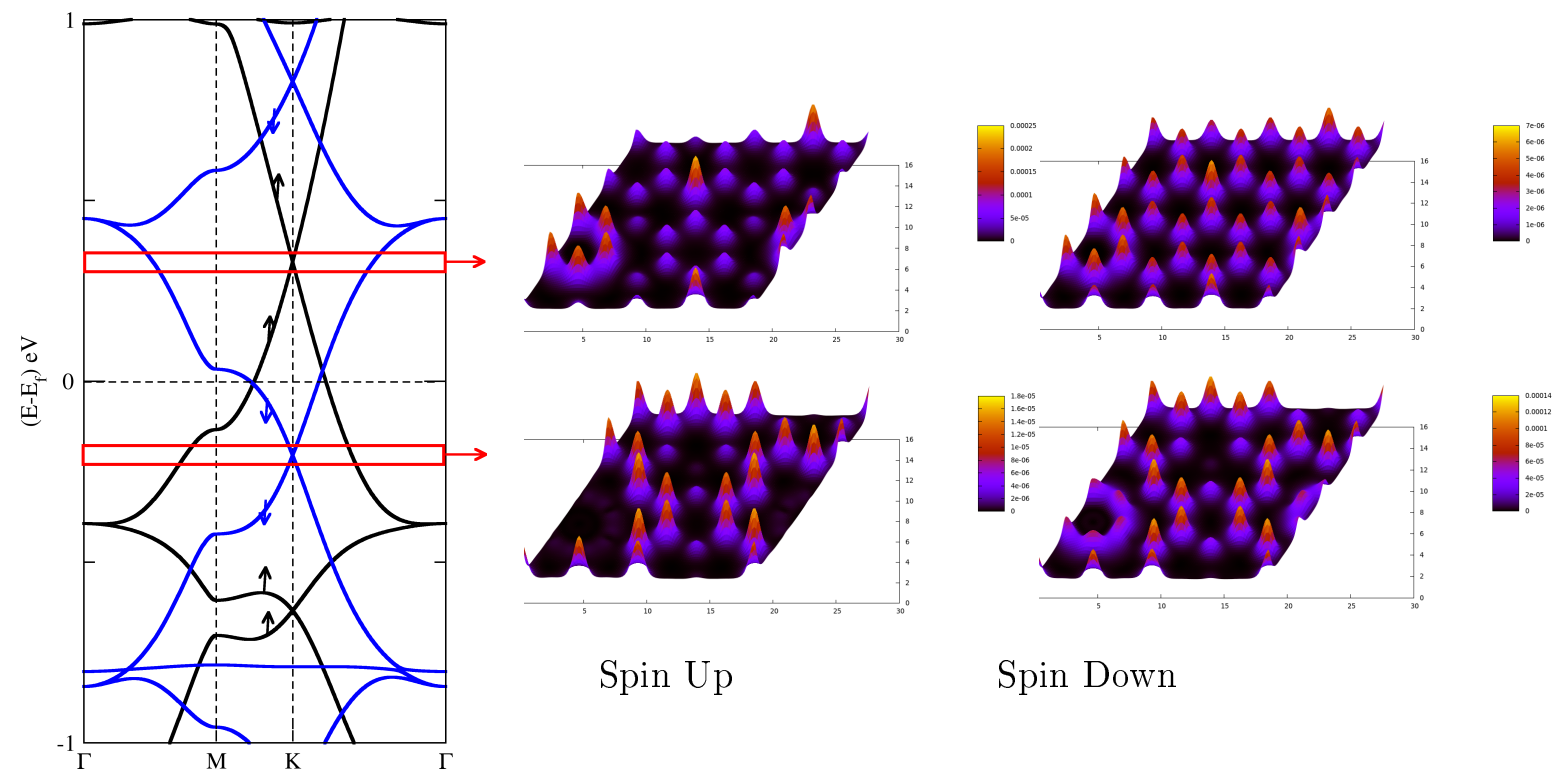

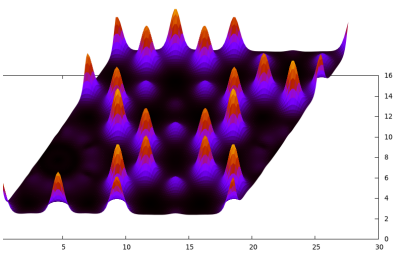

Spin Up

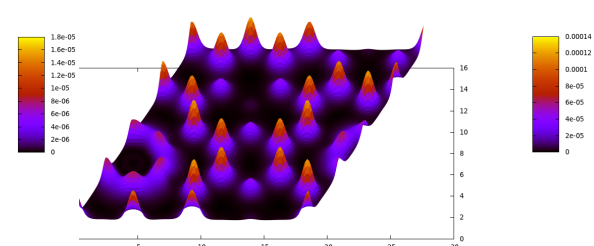

Spin Down

Figura 6.8: Estrutura de bandas da supercélula 4X4. Os retângulos vermelhos ilustram esquematicamente a região de integração das LDOS por spin do lada direito da figura. O intervalo de integração corresponde com $20 \mathrm{meV}$ envolta de cada cone. As LDOS em volta do cone mais externo de estados ocupados (desocupados) obedecem ao mesmo padrão do cone apresentado ocupado (desocupado) próximo ao nível de Fermi.

na família 5x5 em comparação com a família 4x4, para as duas famílias sempre os cones ocupados possuem uma contribuição dos segundos vizinhos do Ru, enquanto que os cones desocupados uma contribuição dos primeiros vizinhos do Ru. Isto contradiz o sentido "comum", dado que espera-se que os primeiros vizinhos sejam mais favoráveis energeticamente do que os segundos vizinhos. Para entender isto, foi estudada uma molécula que possui a mesma simetria local do sistema periódico. Esta molécula formada por 24 átomos de carbono, 12 átomos de Hidrogênio e um átomo de $\mathrm{Ru}$, conforma-se basicamente pela modificação de uma molécula de benzeno, mais precisamente este "benzeno modificado" (BMRu) simula as ligações com os segundos e terceiros vizinhos do $\mathrm{Ru}$, estes últimos ligados a átomos de hidrogênio como representado na figura 6.9a.

É importante salientar que um átomo de $\mathrm{Ru}$ adsorvido na molécula de benzeno não fornece a informação adequada da simetria local do sistema periódico dado que não leva em consideração a contribuição dos segundos vizinhos do Ru, fundamental no entendimento deste sistema. Por isto a necessidade de se estudar o 

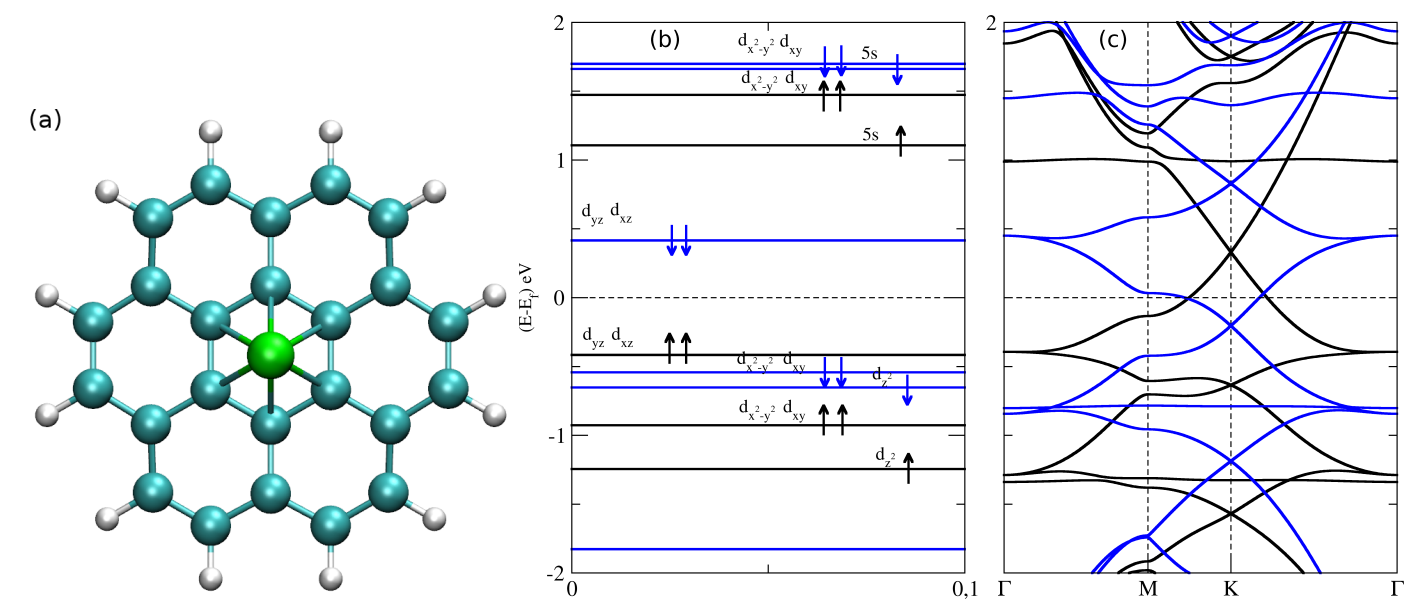

Figura 6.9: (a) Figura esquemática da molécula BMRu. (b) Espectro de energia da molécula BMRu. São representados os estados que contribuem majoritariamente à formação dos níveis de energia nas proximidade do nível de Fermi. O spin $\uparrow$ (Preto) e $\downarrow$ (Azul) dos orbitais $d_{x z}$ e $d_{y z}$ formam o HOMO e LUMO do sistema, como é representado. (c) Estrutura de bandas da supercélula 4X4.

BMRu.

Como é observado na figura $6.9 \mathrm{~b}$, nas proximidades do nível de fermi no ponto $\Gamma$ a estrutura de bandas do sistema periódico apresenta os mesmos níveis de energia que o espectro de energia da molécula $\mathrm{BMRu}$, o que era esperado dado que a simetria local dos dois sistemas é a mesma. Entretanto, observa-se que os estados ocupados nas proximidades do nível de Fermi possuem uma contribuição majoritária dos estados $\mathrm{d}_{x z}$ e $\mathrm{d}_{y z}$ de spin $\uparrow$ e uma dos primeiros vizinhos do $\mathrm{Ru}$ (figura 6.9a), enquanto que os estados acima do nível de fermi possuem uma contribuição majoritária dos estados $\mathrm{d}_{x z}$ e $\mathrm{d}_{y z}$ de spin $\downarrow$ e uma dos segundos vizinhos do átomo de Ru (figura 6.9a), como mostrado na função de onda do HOMO e LUMO (figura 6.10). De fato, quando se aumenta a isosuperfície para realizar o gráfico da função de onda do HOMO e LUMO é possível visualizar a diferença da forma como o $\mathrm{Ru}$ hibridiza com seu vizinhos. Para o estado $\mathrm{d}_{y z} \uparrow$ (HOMO) observa-se que o $\mathrm{Ru}$ hibridiza preferencialmente com os primeiros vizinhos e para o estado $\mathrm{d}_{y z} \downarrow$ (LUMO) preferencialmente com os segundos vizinhos (figura 6.11). Após esta análise, foram estudadas as contribuições dos átomos de carbono à formação dos níveis de energia no ponto $\Gamma$. Foi observado que no ponto $\Gamma$ da estrutura de bandas do sistema 
HOMO
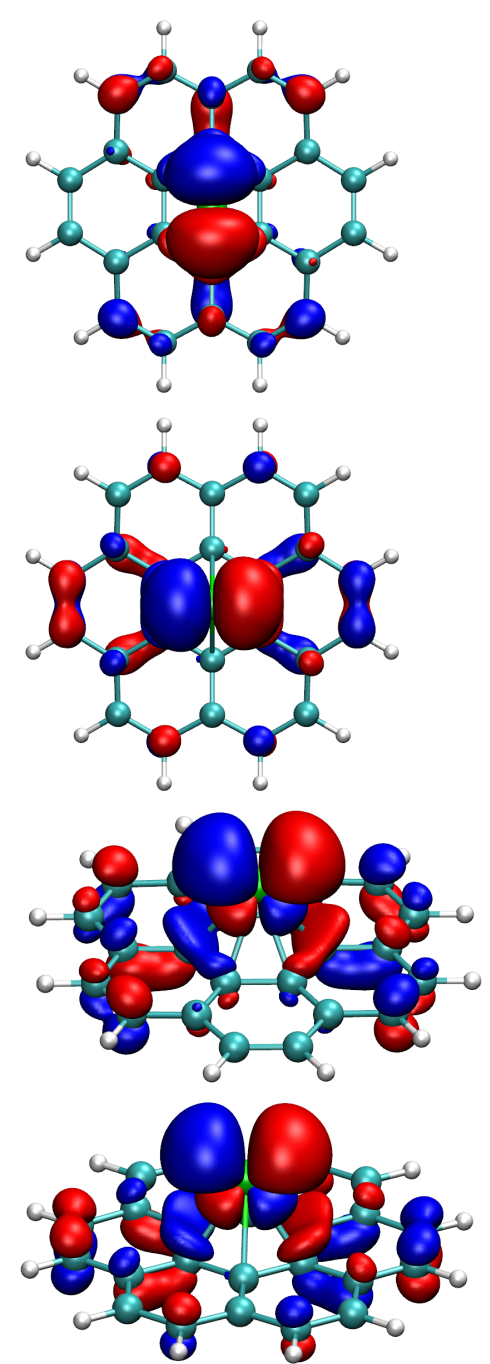

LUMO
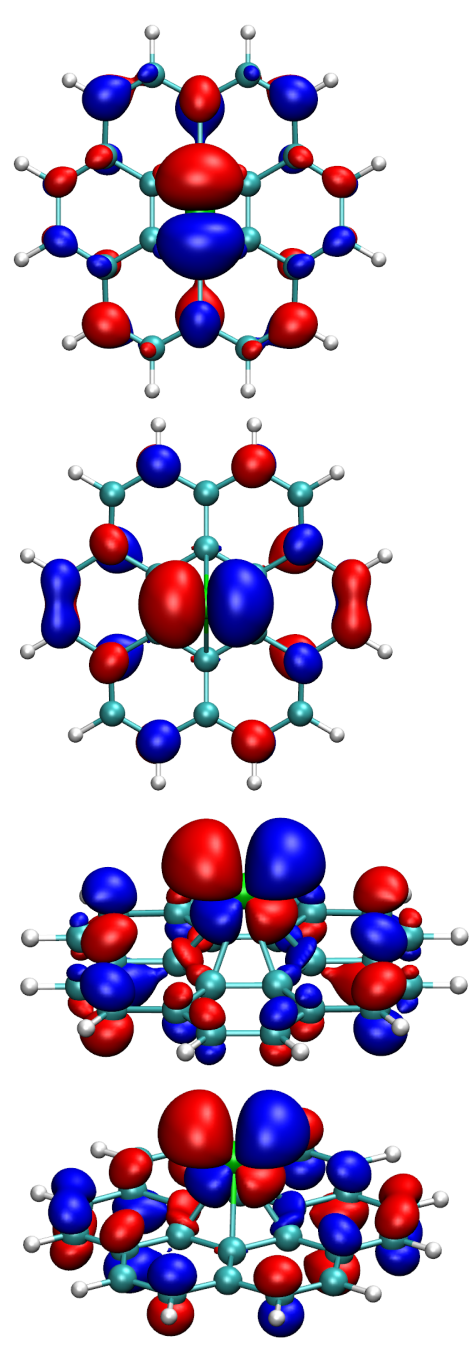

Figura 6.10: Função de onda dos orbitais moleculares HOMO $\left(d_{x z}\right.$ e $\left.d_{y z}-\uparrow\right)$ e do LOMO $\left(d_{x z}\right.$ e $\left.d_{y z}-\downarrow\right)$ da molécula BMRu.

periódico, da mesma forma que o BMRu, os primeiros vizinhos são mais favoráveis energeticamente. Além do mais, tanto a ordem quanto o valor energético dos níveis no ponto $\Gamma$ é a mesma para os dois sistemas como pode ser observado na figura 6.9.

Portanto, a presença dos baricentros e a diferença no comportamento das famílias é regida pela interação do $\mathrm{Ru}$ e seus vizinhos. Em outras palavras, o $\mathrm{Ru}$ fixa as fases da função de onda dos primeiros e segundos vizinhos a qual se propaga através dos carbonos até gerar uma interferência entre a função de onda 


$$
\text { dyz Up }
$$
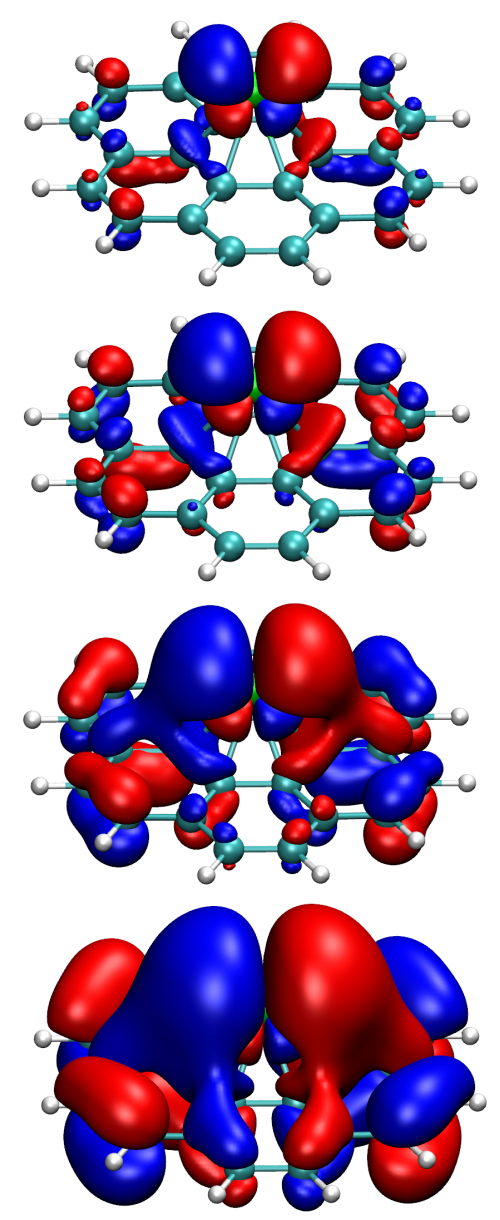

Figura 6.11: Comparação do comportamento entre o HOMO(up dyz) e o LUMO(down dyz) em relação à variação da isosuperfície usada para "plotar" a função de onda.

das diferentes supercélula. Esta interferência leva à existência de regiões com altas e baixas concentrações de estados. Entretanto, a disposição espacial das concentrações de estados eletrônicos e a forte interação do $\mathrm{Ru}$ com o grafeno gera uma dispersão na direção $\Gamma \rightarrow K$ que permite a formação dos cones de Dirac. Nesta visão, uma supercélula de tamanho $(3 n+1) X(3 n+1)$, além de ter picos nos baricentros, apresenta picos da densidade de estados correspondentes à interferência construtiva da função de onda em outras regiões. Ao mapear supercélulas da família 4x4 ao redor de um $R u$, na LDOS da supercélula $(3 n+1) X(3 n+1)$ observam-se picos na densidade de estados localizados nos baricentros das supercélulas mapeadas (3(n1) +1)X $(3(n-1)+1),(3(n-2)+1) X(3(n-2)+1), \ldots, 4 X 4$. Por exemplo, a supercélula de 
tamanho 10X10 apresenta picos correspondentes aos baricentros das geometrias dos tamanhos 4X4 e 7X7, esta última, por sua vez possui picos correspondentes aos baricentros de uma supercélula 4X4, tal como é mostrado na figura 6.12.
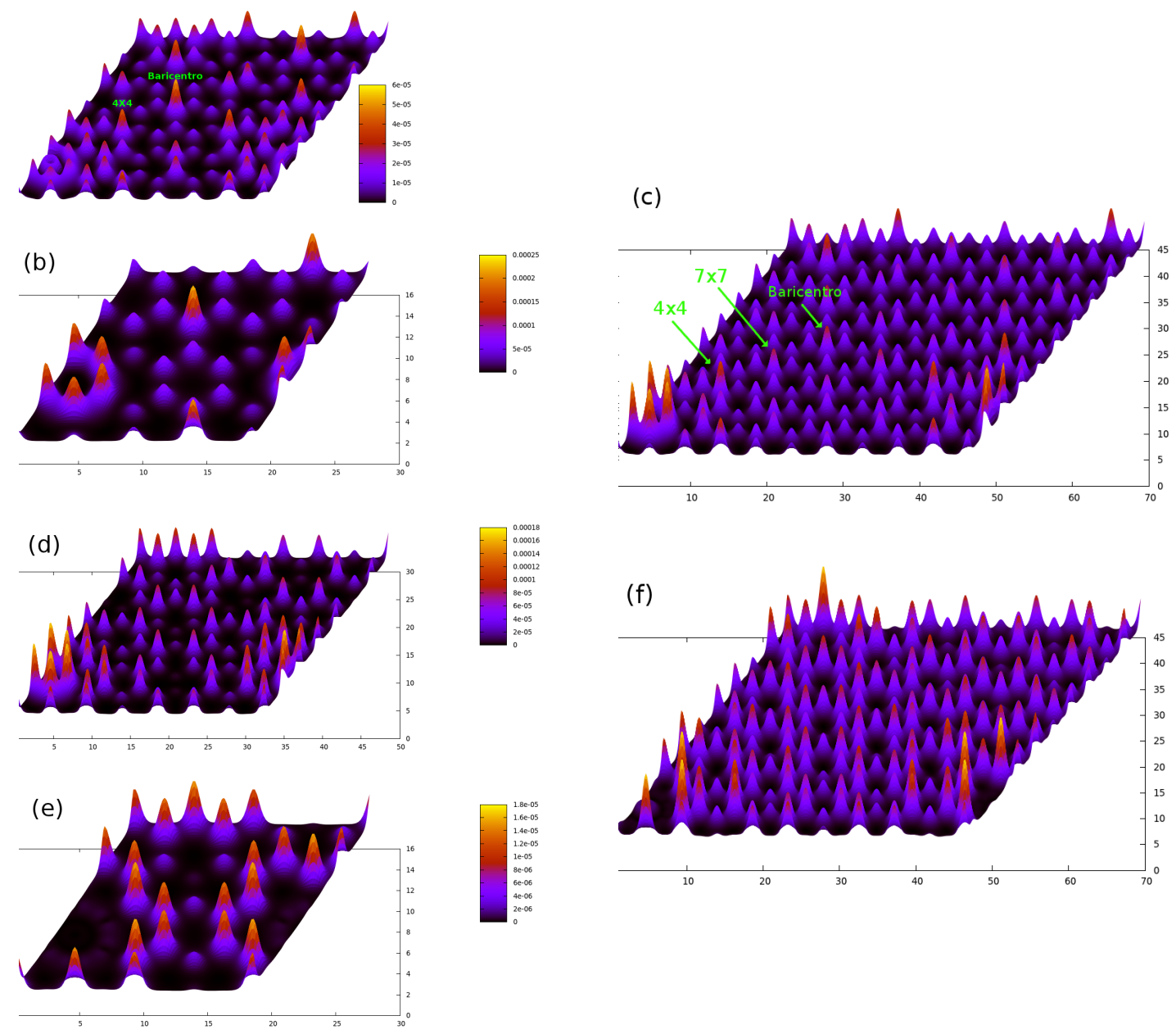

Figura 6.12: Em (a), (b) e (c) se apresentam as LDOS de spin up para as estruturas de bandas das supercélulas $7 \mathrm{X} 7,4 \mathrm{X} 4$ e 10X10 integrando $20 \mathrm{meV}$ envolta do primeiro cone desocupado. Em (d), (e) e (f) se apresentam as LDOS de spin dow para as estruturas de bandas das supercélulas 7X7, 4X4 e 10X10 integrando $20 \mathrm{meV}$ envolta do primeiro cone abaixo no nível de Fermi.

Como consequência da geometria hexagonal do sistema, as superposições das fases das funções de onda fixadas nos primeiros vizinhos resultam em potenciais de baricentros ou antibaricentros dependendo do tamanho da supercélula, mantendo um padrão para cada família de supercélulas. Deste modo, as fases dos primeiros vizinhos que resultam numa interferência construtiva no baricentro das supercélulas $(3 n+1) X(3 n+1)$, nas supercélulas $(3 n+2) X(3 n+2)$ resultam numa interferência 
destrutiva no baricentro. Analogamente, as fases fixadas nos segundos vizinhos (associadas com antibaricentros nas supercélulas $(3 n+1) X(3 n+1)$ ) resultam em uma superposição construtiva no baricentro para as supercélulas $(3 n+2) X(3 n+2)$. Contudo, embora a LDOS das supercélulas 3nX3n apresentem picos nos primeiros e segundos vizinhos (figura 6.6), nestas supercélulas o centro geométrico do triangulo de Ru situa-se no meio de um hexágono de carbonos, de modo que não é possível a formação de interferências construtivas (ou destrutivas) que gerem baricentros e antibaricentros.

O fato das supercéclulas 3nX3n não permitirem a existência de baricentros e das suas estruturas de bandas não possuírem cones induz a pensar que de forma efetiva os baricentros são responsáveis pela existência do comportamento de férmions de Dirac sem massa. Neste trabalho foram feitos os cálculos necessários para mostrar que os baricentros "de forma efetiva" geram os cones da estrutura de bandas. 


\subsection{1 Átomo de hidrogênio no baricentro}

Ao colocar um átomo de hidrogênio acima do átomo de carbono no qual situa-se o baricentro, os cones associados com a contribuição dos baricentros desaparecem da estrutura de bandas. Na figura 6.13 é apresentada a geometria 4X4 como um exemplo deste comportamento. Os cones que são gerados pelos picos da densidade de estados localizados nos antibaricentros são perturbados pela presença do H.
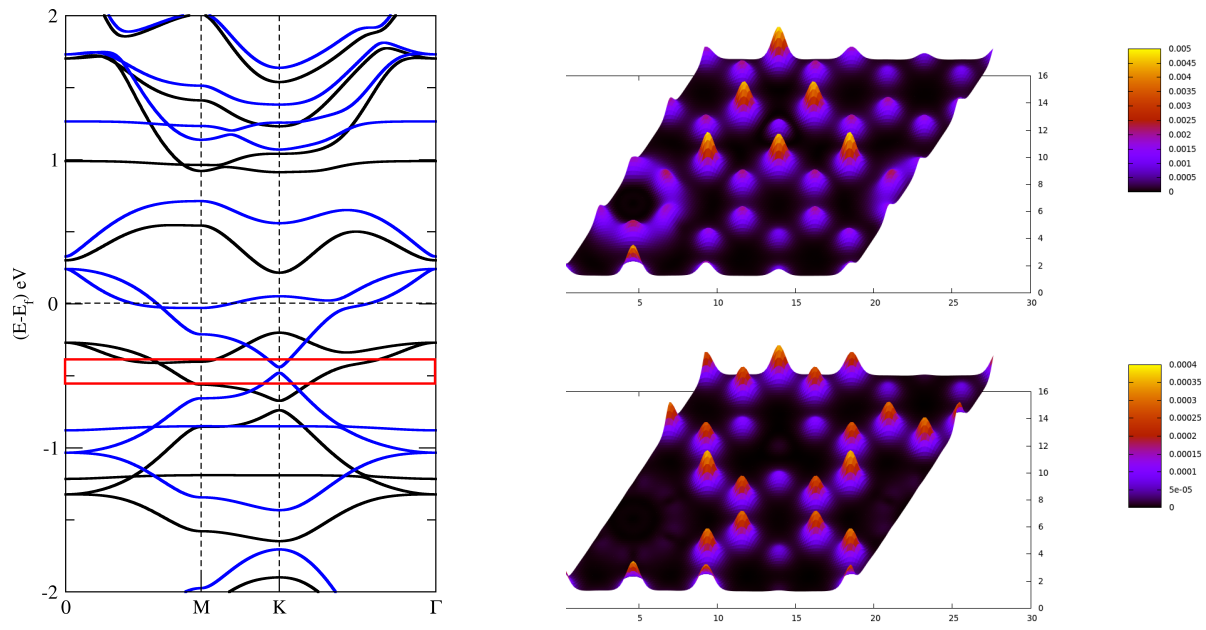

Figura 6.13: Estrutura de bandas do $\mathrm{Ru}$ quando adsorvido na supercélula 4X4 com um átomo de hidrogênio no carbono no qual situa-se o baricentro. As LDOS corresponde à região de integração representada no retângulo vermelho com a LDOS associada ao spin $\uparrow$ na parte superior e a LDOS associada ao spin $\downarrow$ na prte inferior.

\subsubsection{STM}

Embora estes resultados demonstrem a existência de uma distribuição de baricentros com simetria hexagonal, eles não permitem "visualizar uma rede"com vértices nos baricentros. Em princípio, fazer uma imagem de STM é a forma adequada de determinar se existe comunicação entre os baricentros via uma rede hexagonal, mas um limitador encontrado no código SIESTA é justamente que ele não permite fazer este tipo de imagens quando tem-se uma rede sem vetores primitivos de traslação ortogonais, como é o caso do sistema estudado. Usar outro código 
que leve em conta a interação spin-órbita e permita a construção destas imagens possibilita contornar esta dificuldade. Foi usado o código VASP, além de fazer-se as imagens de STM usando este código, foram confirmados os resultados obtidos usando a implementação SIESTA-SOC, obtendo-se uma concordância absoluta. A imagem de STM do sistema com spin-órbita a $0,2 \mathrm{eV}$ embaixo do nível de Fermi mostra claramente nas regiões vermelhas os átomos de Rutênio e coloca em evidência a rede hexagonal de baricentros.

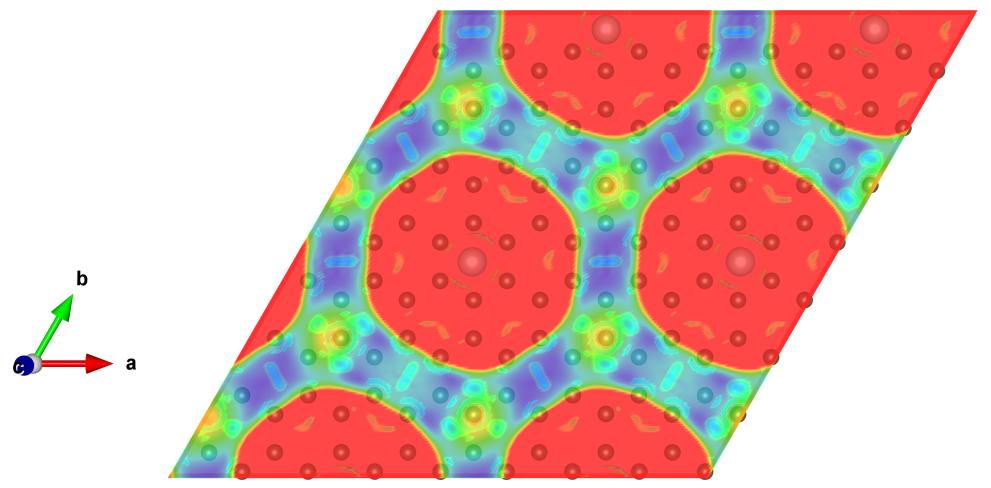

Figura 6.14: Imagen STM da rede hexagonal de baricentros para uma supercélula $4 \mathrm{X} 4$.

\subsubsection{Rede triangular de $\mathrm{Ru}$}

Espera-se que a quebra da simetria triangular da distribuição espacial dos átomos de $\mathrm{Ru}$ no grafeno leve a uma mudança espacial dos baricentros. De forma que, se a existência da rede hexagonal dos baricentros determina a presença dos férmions de Dirac sem massa, quando eliminada a configuração triangular dos $R u$, os baricentros deixem de formar uma rede hexagonal e portanto, os cones de Dirac desapareçam. Ao construir uma rede triangular de $\mathrm{Ru}$ acima do grafeno verifica-se que este sistema apresenta cones de Dirac. Enquanto que se os Ru formam uma rede que não tem simetria triangular, os "baricentros" descrevem uma estrutura com uma simetria diferente à associada a uma rede hexagonal e portanto, o comportamento de férmions de Dirac sem massa desaparece. 
Usando 3 átomos de $\mathrm{Ru}$ por célula unitária, construímos uma rede triangular de $\mathrm{Ru}$ acima do grafeno equivalente a uma célula 4x4 de grafeno (figura 6.15). Verifica-se a presença dos cones de Dirac no ponto $\Gamma$ da zona de Brillouin, da mesma configuração de spin mostrada no ponto $K$ da estrutura de bandas do Ru adsorvido numa supercélulas 4X4 de grafeno. Quando os Rutênios são
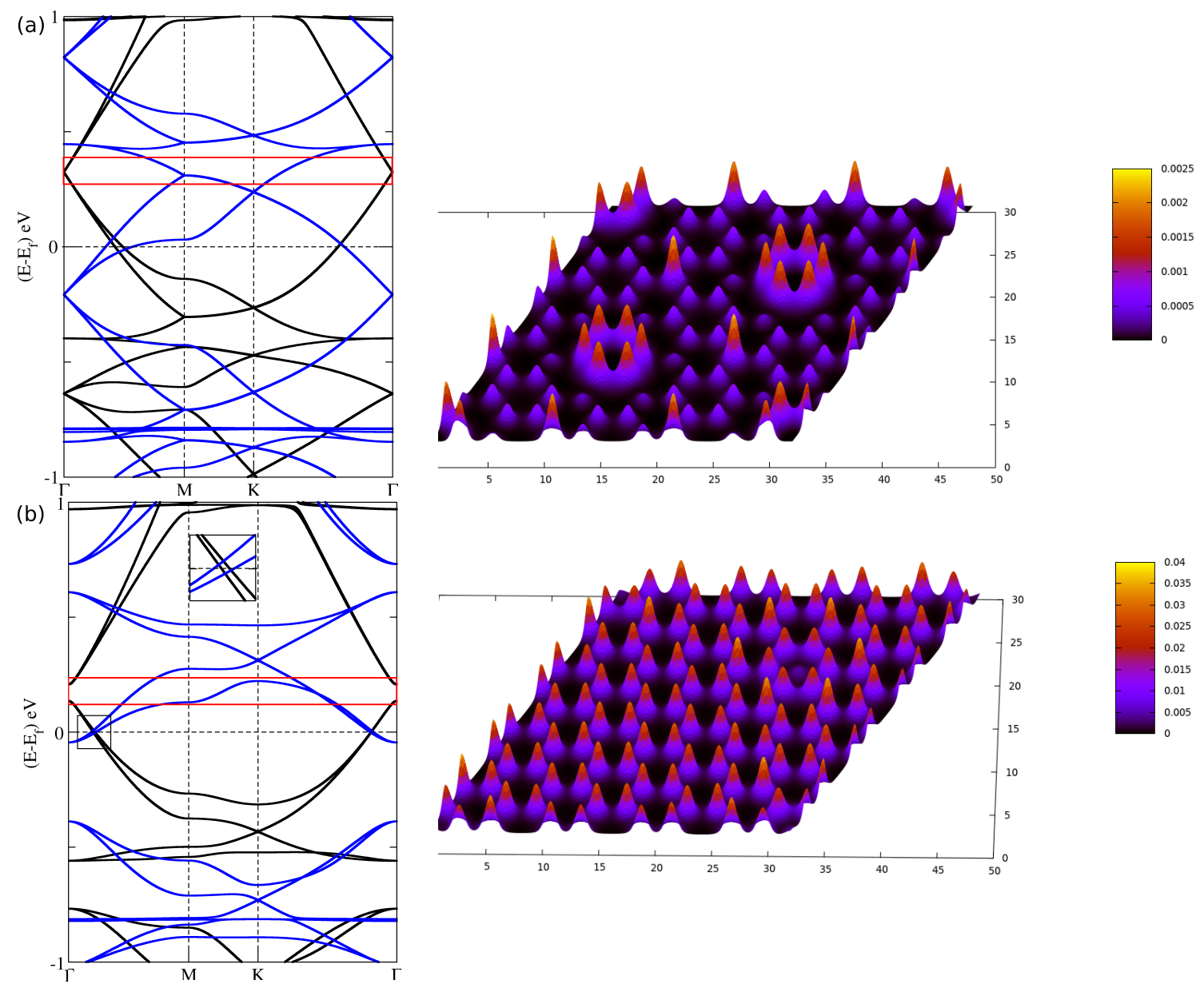

Figura 6.15: Estrutura de banda do Ru adsorvido em grafeno. (a) Quando os Ru são depositados em uma configuração triangular. A LDOS está associada aos estados com spin $\uparrow$ (cor preta) no retângulo vermelho. (b) Quando os Ru são depositados em uma configuração hexagonal. A LDOS está associada aos estados com spin $\uparrow$ (cor preta) no retângulo vermelho. O requadro é um zoom nos estados que cruzam no nível de Fermi. Este zomm evidencia que não há formação de cone de Dirac.

adsorvidos numa configuração hexagonal, os centros geométricos destes ficam em uma distribuição com simetria triangular e verifica-se que não há presença de cones. A presença dos baricentros é percebida quando se "plota" a densidade de estados localizada do arranjo de Ru com simetria triangular (figura 6.15). As densidades 
de estados localizadas deixam claro que os baricentros associados à distribuição triangular dos Ru existem e há uma relação entre a existência de baricentros com a distribuição hexagonal e a presença do comportamento de férmions de Dirac sem massa na estrutura de bandas.

\subsubsection{Rede retangular de $\mathrm{Ru}$}

Espera-se que os Ru quando adsorvidos em uma simetria retangular gerem uma estrutura de bandas sem a presença dos quatro cones de Dirac como acontece no sistema com $\mathrm{Ru}$ dispostos em uma configuração com simetria triangular. Após a construção e relaxação do sistema obtem-se o resultado esperado como é mostrado na figura 6.16. Ao construir a rede retangular de $\mathrm{Ru}$ inicialmente foi considerada a mesma distância Ru-Carbono usada nos testes anteriores. Entretanto, após a relaxação a distância Ru-Carbono aumenta aproximadamente 5\%, adicionalmente, a configuração mais estável para os $\mathrm{Ru}$ é a triangular dado que possui menor energia total. Portanto, dado que a presença de cones de Dirac é devida única e exclusivamente à existência de uma rede hexagonal de baricentros, espera-se que experimentalmente os $\mathrm{Ru}$ fiquem em uma disposição triangular e seja possível a observação do comportamento particular deste sistema.

Os testes feitos permitem afirmar que por meio da adsorção de Ru em uma configuração triangular no grafeno gera-se um sistema de baricentros e antibaricentros com simetria hexagonal que efetivamente geram cones de Dirac. Neste trabalho encontrou-se que estes sistemas apresentam sinais fortes de fases topológicas. 

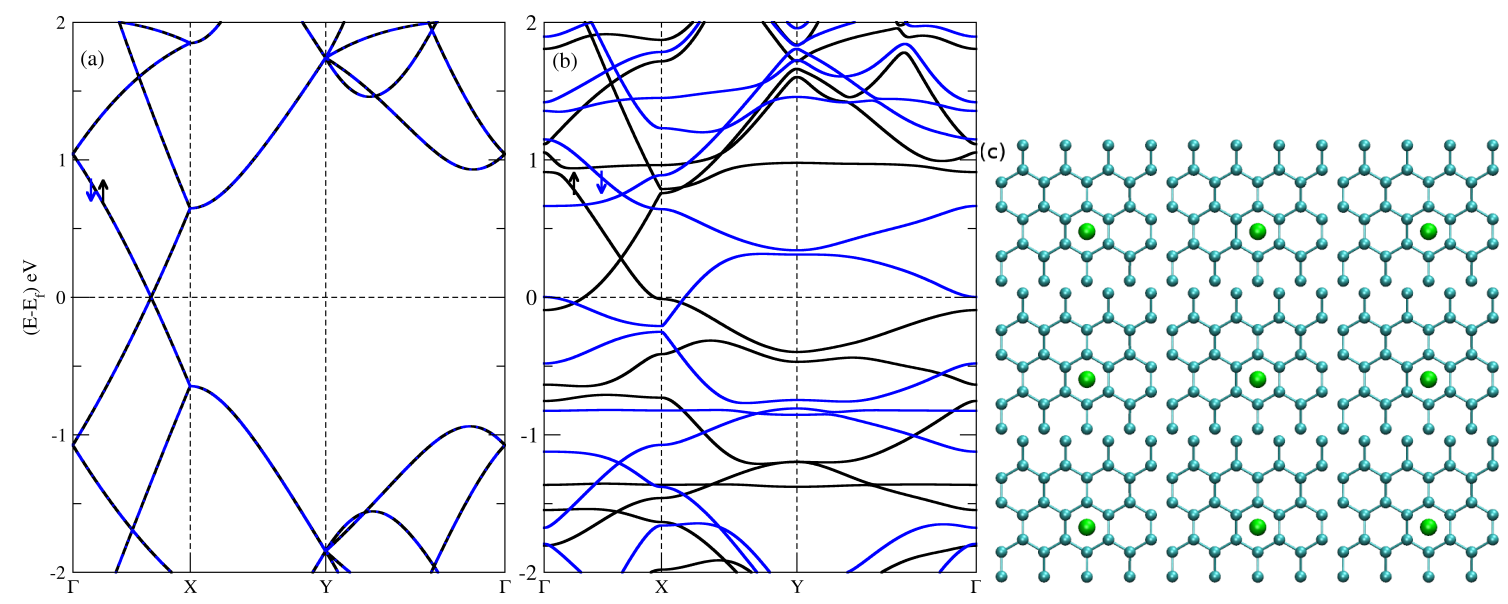

Figura 6.16: (a) Estrutura de bandas do grafeno pristino considerando uma célula unitária retangular. (b) Estrutura de bandas de uma distribuição retangular de Ru depositada no grafeno, esquematizada na figura (c).

\subsection{Efeito Hall quântico anômalo em baricentros}

No QSH o SOC intrínseco permite gerar uma corrente de spin transversal como resposta quantizada à presença de um campo elétrico. Este fenômeno requer a conservação da simetria RT quebrada por campos magnéticos externos ou momentos magnéticos internos tal como a contribuição SOC Rashba. Apesar do SOC Rashba prejudicar o efeito QSH, este ajuda a realizar outro fenômeno topológico importante denominado efeito Hall quântico anômalo (QHA). Este efeito é sinalizado por uma carga quantizada associada à condutância Hall para um estado isolante. De modo diferente ao QSH, o efeito QHA requer a quebra da simetria TR e ao contrário de efeito QH que é induzido por um campo magnético forte, o efeito QHA depende da magnetização interna e do SOC. O valor quantizado da condutância Hall está relacionada ao número topológico do "bulk"denominado número de Chern, sua robustez à desordem e outras perturbações[2].

No QHA abre-se um gap no "bulk"quando o SOC Rashba e o potencial de troca estão presentes. A presença deste gap indica um estado isolante. Segundo Qiao et al.[75], este estado isolante é topologicamente não trivial dado que possui estados de borda quirais que exibem uma condutância Hall de carga quantizada. 
De modo que, a curvatura Berry culmina em um pico nas esquinas da primeira zona de Brillouin como o mesmo sinal. Por exemplo, em uma rede hexagonal a curvatura de Berry tem o mesmo sinal nos pontos não equivalentes $K$ e $K^{\prime}$ já que a rede hexagonal preserva a simetria de inversão bidimensional. Em contraste com o "valley Hall effect" encontrado no grafeno com quebra de simetria entre as subredes A e B, onde a curvatura de Berry nos pontos $K$ e $K^{\prime}$ tem sinais opostos[76].

Qiao et al.[75], predisseram que o efeito Hall quântico anômalo podia ser realizado em grafeno introduzindo SOC Rashba e um potencial de troca e mostraram a evolução das estruturas de bandas com a presença do potencial de troca e SOC Rashba, característica de um QHA (figura(6.17)). O aparecimento de estados de borda quirais no gap do "bulk" está intimamente relacionado com propriedades topológicas caracterizadas pela condutância Hall de carga. No artigo de Qiao et al.[75], é reportado um valor de $\sigma_{y x}=C e / h$, neste caso o número de Chern $C$ é igual a 2 dado que existem dois canais de condução cada um com diferente spin .

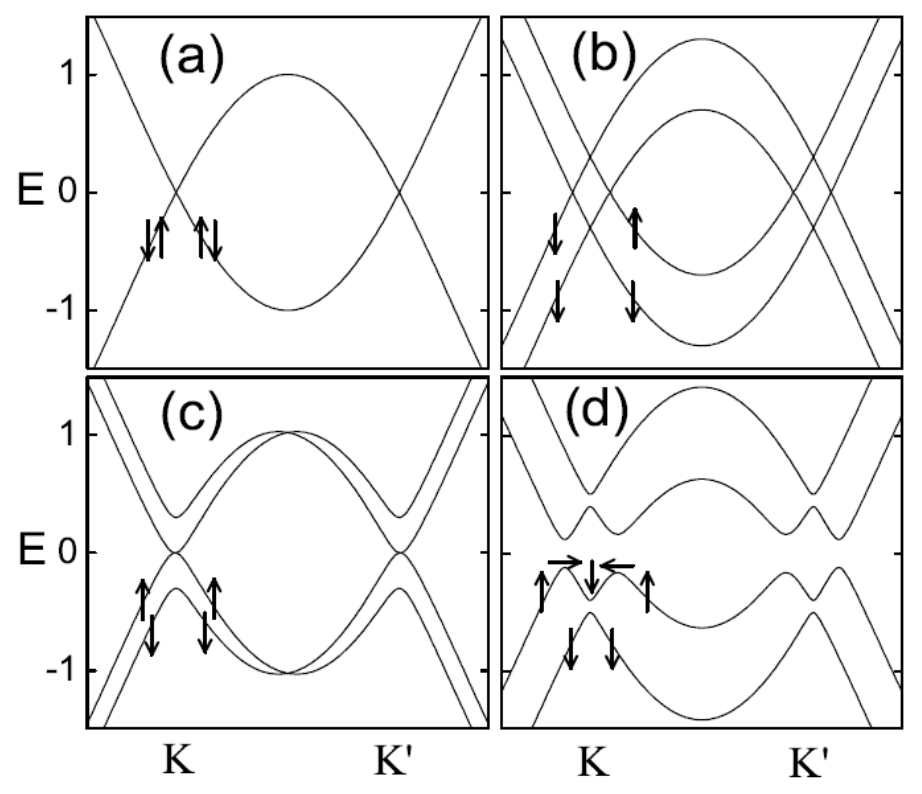

Figura 6.17: (a)estrutura de banda do grafeno pristino com cones de Dirac centralizados nos pontos $K$ e $K^{\prime}$ no espaço recíproco. (b)Quando unicamente o potencial de troca é aplicado. (c) Quando só o SOC Rashba é aplicado, os estados de spin são misturados e a degenerescência de spin é levantada. (d) Um gap de "bulk" é aberto quando tanto o SOC Rashba e o campo de intercâmbio estão presentes. 
Qiao et al.[75], mostraram que para um dado nível de Fermi, existem quatro diferentes estados de borda marcados como $A, B, C$ e $D$ figura a. Dado que $v(k)=\frac{1}{\hbar} \frac{\partial E(\kappa)}{\partial \kappa}$, os estados $A$ e $B(C$ e $D)$ propagam-se ao longo da mesma direção $-x(+x)$. Na figura 6.18b, são representadas as distribuições de onda $|\psi|^{2}$ dos quatro estados a través da amplitude, pode-se observar que as funções de onda dos estados $A$ e $B$ estão localizados no limite esquerdo enquanto que as funções de onda dos estados $C$ e $D$ são essencialmente zero nesta região. Em outras palavras, o efeito QHA é caracterizado por apresentar dois canais do mesmo spin em cada borda. Deste modo, o efeito QHA apresenta uma configuração de spin nas proximidades do nível de Fermi da estrutura de bandas como a encontrada no sistema composto por um átomo de $\mathrm{Ru}$ depositado em uma supercélula de grafeno com tamanho $(3 n+1) X(3 n+1)$ e $(3 n+2) X(3 n+2)$, como é ilustrado na figura 6.18d.
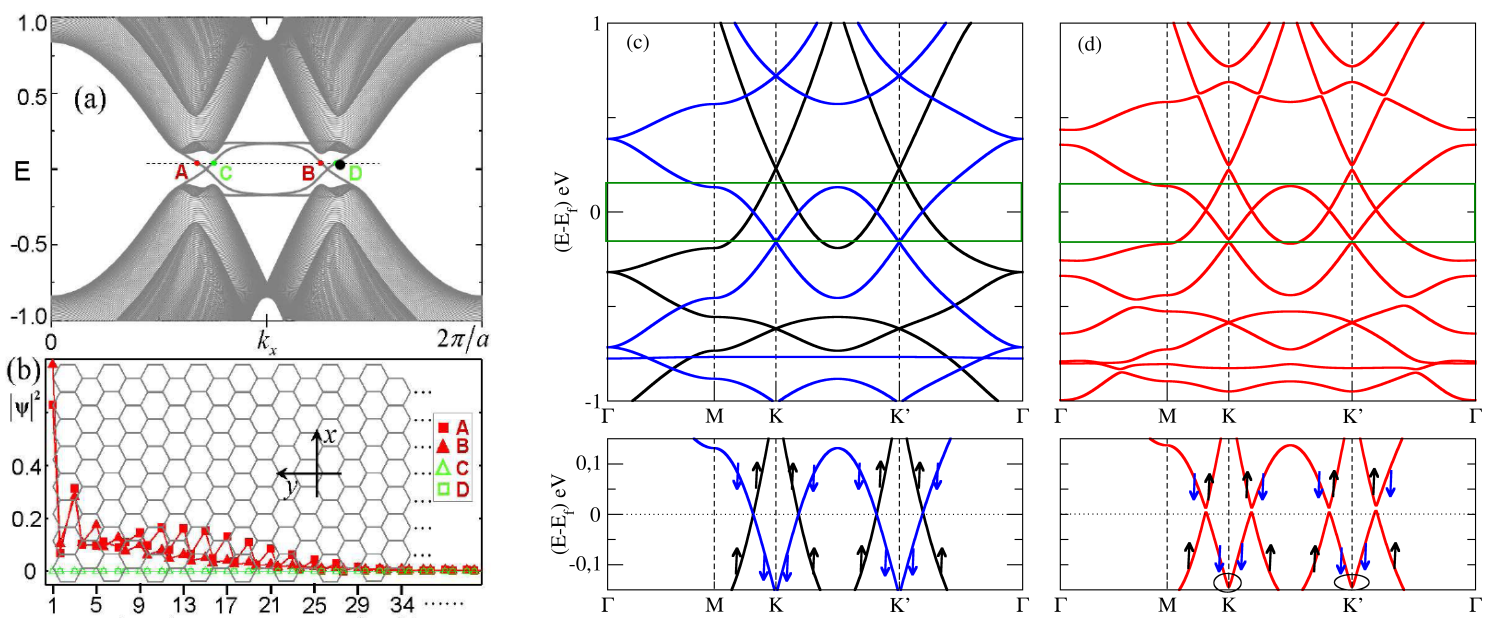

Figura 6.18: (a) Estados de borda marcados como $A, B, C$ e $D$ (a). (b) Distribuições de onda $|\psi|^{2}$ dos quatro estados a través da amplitude (só parte das fitas com uma borda zigzag são observáveis). (c) Estrutura de bandas levando em conta a polarização de spin, são especificadas as direções do spin no retângulo. A mudança da estrutura de bandas com SOC (d) é caraterística do QHA e a disposição do spin mas proximidades do nível de Fermi.

A estrutura de bandas da rede hexagonal de baricentros e antibaricentros, quando se considera o SOC, apresenta uma evolução característica de um QHA e as componentes do spin seguem a mesma sequência apresentada na figura 6.17. Além do mais, a magnetização interna do sistema é diferente de zero de forma que verifica- 
se a quebra da simetria de reversão temporal característica principal do QHA. Em outras palavras, a estrutura de bandas da rede hexagonal de baricentros evidencia a presença de características de uma fase topológica associada com estados topológicos quirais de um QHA.

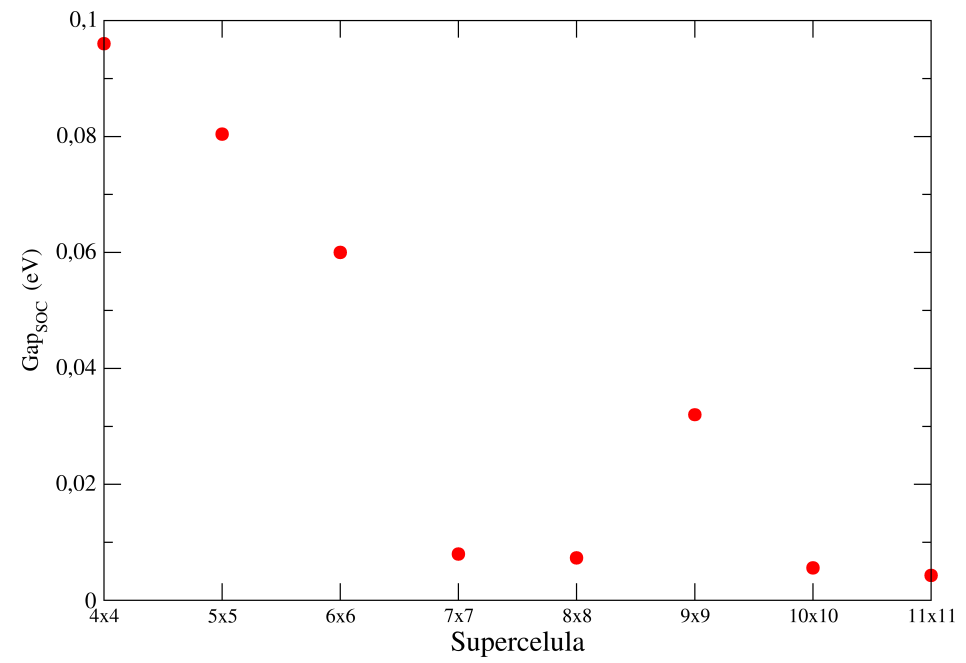

Figura 6.19: Variação do Gap SOC em relação ao tamanho das supercélulas.

O gap gerado pelo SOC foi calculado usando o SIESTA-SOC e encontrouse o valor de $90 \mathrm{meV}$ na supercélula $5 \times 5$. O gap diminui conforme decresce a concentração de $\mathrm{Ru}$ no sistema mantendo uma magnetização diferente de zero. Entretanto, a pertubação do SOC Rashba do Ru gera um gap no sistema, de forma que incluso para baixas concentrações, a estrutura de bandas apresenta um pequeno gap (figura 6.19). Isto pode ser entendido como uma variação adiabática do sistema de baricentros ao grafeno. Para baixas concentrações de $\mathrm{Ru}$ o sistema atinge o limite estudado por Qiao et al.[75], no qual o grafeno é perturbado somente pelo SOC do metal de transição com momento magnético diferente de zero. O aumento da distância Ru-Ru resulta na diminuição da superposição das fases, de modo que a característica do sistema muda até perder o caráter de um sistema de baricentros, de forma que, o comportamento do grafeno predomina, porém sem fechar o gap nessa transição. Dado que fases topológicas da mesma classe podem ser conectadas mediante transições adiabáticas sem fechar o gap espectral, pode-se afirmar que a 
rede hexagonal de baricentros e antibaricentors é fase topológica QHA.

Por outra parte, dado que o grafeno pristino e a rede hexagonal de baricentros fazem parte de duas classes topológicas diferentes, espera-se que ao fazer uma variação adiabática para levar o sistema de baricentros ao grafeno o gap feche. Consequentemente, neste trabalho foi afastado o Ru lentamente do plano do grafeno, observando a variação da estrutura de bandas foi verificada a existência de um gap nulo nessa transição. A aparição deste estado metálico pode ser associada com a transição de uma fase topológica QHA a uma fase trivial. Para desenvolver este estudo foi variada a distância do $\mathrm{Ru}$ em relação ao plano do grafeno, sem relaxar a estrutura, iniciando da posição de equilíbrio 1.683 Ang. Na figura 6.20 é mostrado que quando o Ru está afastado do plano do grafeno $1.983 \AA{ }^{3}$ aparece um gap nulo ${ }^{4}$. O argumento exposto de transições adiabáticas tem sido usado para a caraterização da fase topológica de outros materiais.
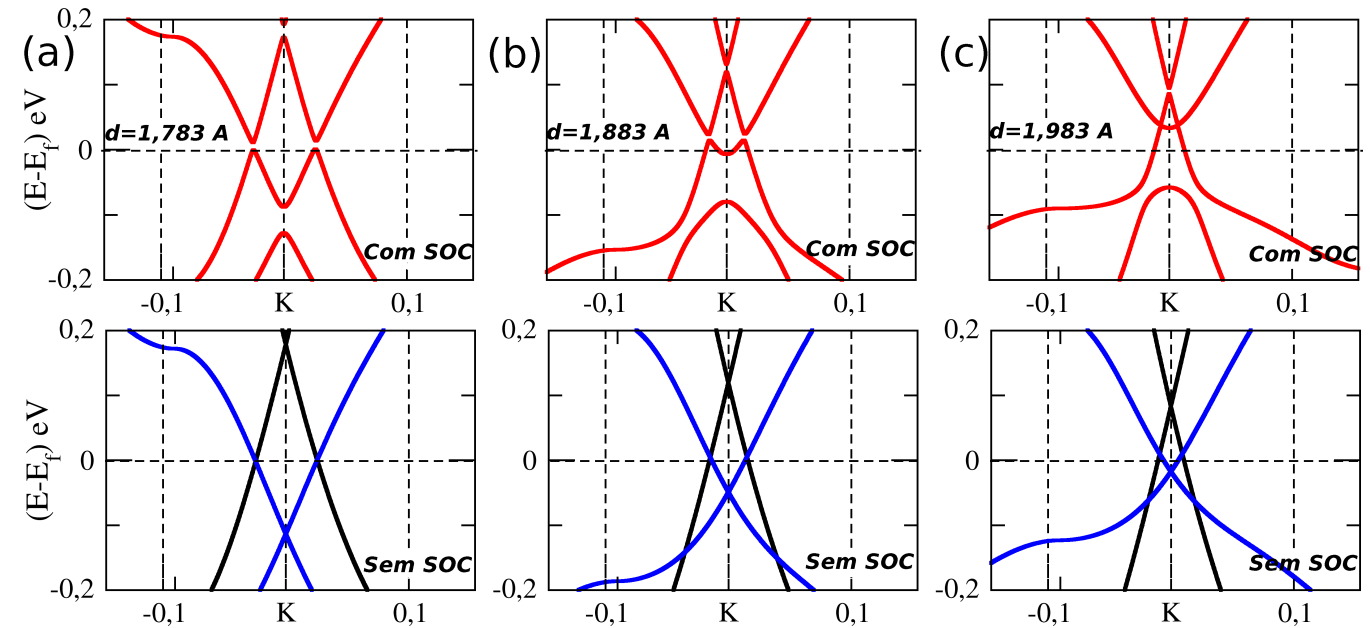

Figura 6.20: Em (a), (b) e (c) são apresentadas a estruturas de bandas para as distância Ru-grafeno de 1.783, 1.883 e 1.983 Ang. Embaixo da estrutura de bandas levando em consideração o SOC (cor vermelhar) é apresentada a estrutura de bandas sem SOC, especificando o spin $\downarrow$ pela cor azul e o spin $\uparrow$ pela cor preta.

É importante notar que a proposta original do QHA associa-se ao comportamento do grafeno na presença de um forte SOC e um potencial de troca,

\footnotetext{
${ }^{3} \mathrm{o}$ qual corresponde com uma distância de $17.83 \%$ vezes maior à do sistema em equilíbrio

${ }^{4}$ É importante notar que existe um efeito de transferência de carga, de forma que o nível de fermi calculado é errado. No entanto, isto não afeta a análises qualitativa feita.
} 
enquanto que neste trabalho a proposta é baseada em um sistema com caraterísticas fundamentalmente diferentes às do grafeno pristino e que intrinsecamente apresenta características dos sistemas com estados QHA tais como um forte potencial de troca, forte SOC e magnetização liquida diferente de zero.

\subsection{QSH em baricentros}

Foi mostrado que o Ru hibridiza fortemente quando é adsorvido no sítio B do grafeno, de modo que fixa as fases da função de onda de seus primeiros e segundo vizinhos. A propagação destas fases via grafeno resulta na superposição construtiva das funções de onda de cada supercélula, gerando redes de baricentros e antibaricentros com simetria hexagonal. Cada uma desta redes apresenta um cone Dirac para cada componente do spin. Desta forma, a estrutura de bandas deste sistema possui quatro cones de Dirac. Na presença do SOC a estrutura de bandas fica em um formato próprio de um sistema QHA. Adicionalmente, para gerar um estado QSH no grafeno com átomos adsorvidos, tem-se que perturbar o grafeno somente pela transferência de SOC do átomo depositado. Em outras palavras, a formação de baricentros e antibaricentros precisa de uma forte perturbação das propriedades eletrônicas do grafeno, enquanto que a fase QSH neste material é construída somente pela transferência do SOC sem modificar fortemente as propriedades eletrônicas. Assim, gerar uma fase QSH em um sistema de baricentros pela deposição de átomos pesados em grafeno é fisicamente contraditório. Além do mais, a partir de uma intensa procura determinou-se que muitos átomos quando adsorvidos no grafeno induzem um momento magnético no sistema, de forma que a simetria de TR é quebrada.

Por outra parte, a posição B no grafeno é energeticamente mais favorável para a adsorção de átomos de $\mathrm{Ba}$ e $\mathrm{Sr}$ no grafeno ${ }^{5}$. Os estados destes metais

\footnotetext{
${ }^{5}$ De acordo com o modelo de Kane e Mele[3] átomos adsorvidos na posição B no grafeno
} 
pesados não hibridizam fortemente com o grafeno, de modo que não permitem a formação de redes de baricentros e antibaricentros com simetria hexagonal. Entretanto, as propriedades eletrônicas são perturbadas única e exclusivamente pelo SOC transferido na adsorção do átomo. Foram feitos cálculos de estrutura eletrônica de um único átomo adsorvido numa supercélula de grafeno dos tamanhos 4x4, 5x5 e 6x6. O cálculo de estrutura eletrônica tanto para o Ba quanto para o Sr evidencia que o formato da banda não foi modificadas pela adsorção do átomo. Entretanto, o gap aumenta 1000 vezes o valor do gap no grafeno pristino. No caso do Sr o gap SOC é de $0,8 \mathrm{meV}$, enquanto que a estrutura de bandas $\mathrm{Ba} / \mathrm{Gr}$ exibe um gap de 1,2 meV como é observado na figura 6.21e , já que o gap é subestimado pelo uso do potencial de troca e correlação LDA, esperam-se que os valores sejam maiores com o uso de outras abordagens. Como foi explicado, a figura $6.21 \mathrm{f}$ evidencia que a formação do cone de Dirac neste sistema não está associada com a presença de picos na densidade de estados localizados nos baricentros ou antibaricentros.

Embora neste trabalho não foi calculado o invariante topológico $Z_{2}$, podese usar o argumento da variação adiabática para determinar a classificação topológica de um sistema. Se ao variar adiabaticamente o hamiltoniano do sistema o gap espectral é diferente de zero para todos os hamiltonianos resultantes da variação, estes representam sistemas que fazem parte da mesma classe topológica. Este argumento permite determinar a classificação topológica de um sistema se é possível conectá-lo adiabaticamente a outro sistema com uma fase topológica conhecida. Motivados por este princípio foram feitos dois tipos de variações no hamiltoniano; o átomo adsorvido foi afastado lentamente e foi mudada a intensidade do SOC deste átomo. Nos dois casos observa-se que o gap não fecha, o qual prova cabalmente que o Ba e o Sr quando adsorvidos no grafeno apresentam estados QSH. Weeks et al.[64], reportaram dois átomos que quando adsorvidos no grafeno apresentam QSH, em permitem mediar a dependência do termo de "hopping" com os segundos vizinhos em relação ao SOC. 

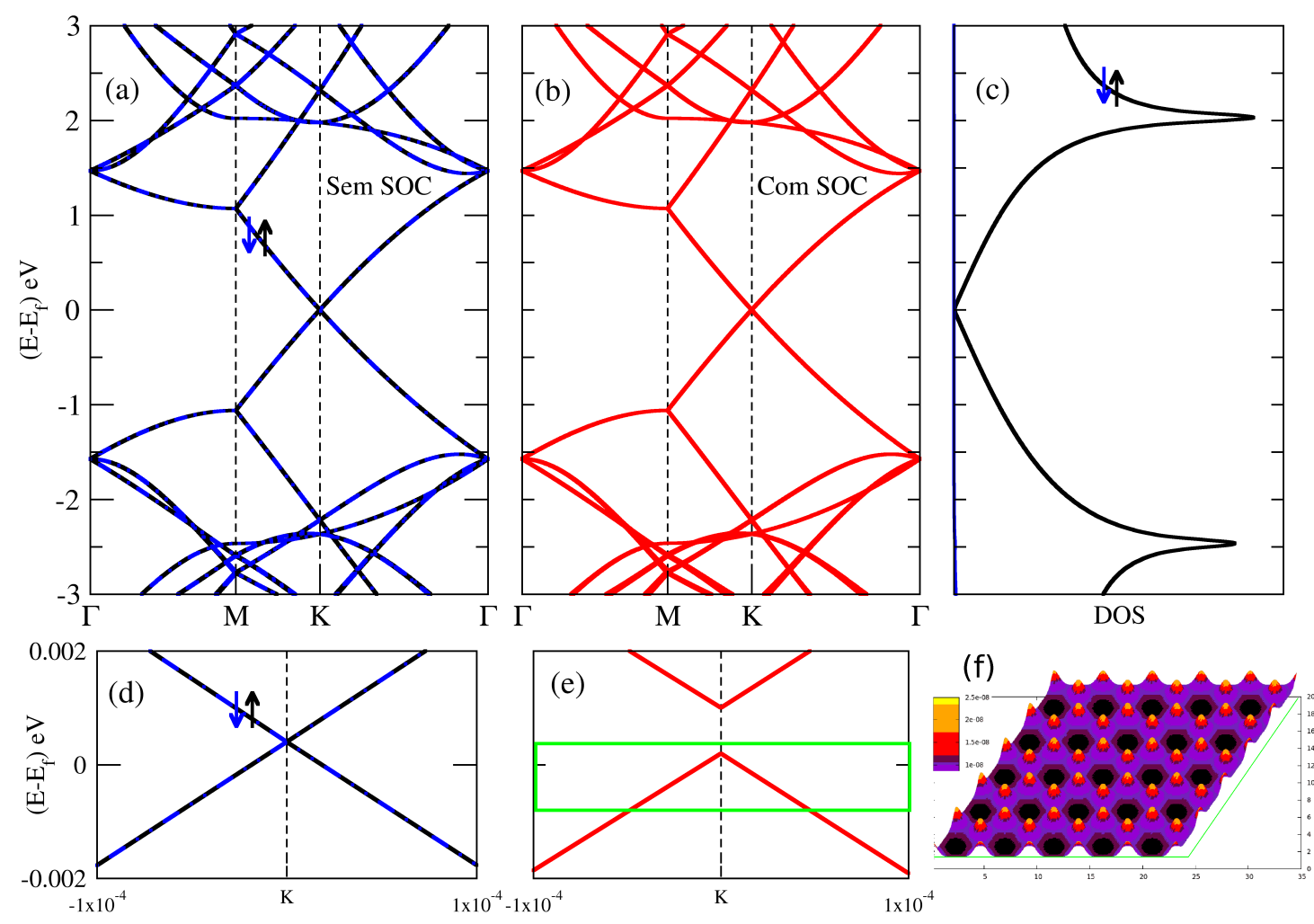

Figura 6.21: Transferência do SOC do Ba ao grafeno. (a) Estrutura de bandas sem considerar SOC. A cor azul representa o spin down e a cor preta o spin up. Em (b) é mostrado que a estrutura de bandas do não apresenta fortes perturbações associadas com a inclusão do SOC representado pela cor vermelha. Entretanto, quando se observa a estrutura de bandas nas proximidades do cone sem considerar o SOC (d), evidenciam-se os efeito do SOC como apresentado em (e). (f) LDOS na região de integração representada esquematicamente pelo retângulo verde em (e).

princípio, mais notório do que no grafeno pristino: o Indio e o Talio. Entretanto, não tem sido reportado que que o Ba e o Sr também apresentam esta propriedade, como foi mostrado neste trabalho. A título de exemplo, serão mostrados os resultados da variação adiabática para o caso do átomos de Ba.

Foi aumentado o SOC do carbono ao dobro para conseguir um gap de 0,4 meV no grafeno pristino, de forma que a variação do hamiltoniano é feita até atingir este valor no gap. Entretanto, esta abordagem não afeta muito o valor inicial do gap com os metais adsorvidos. 

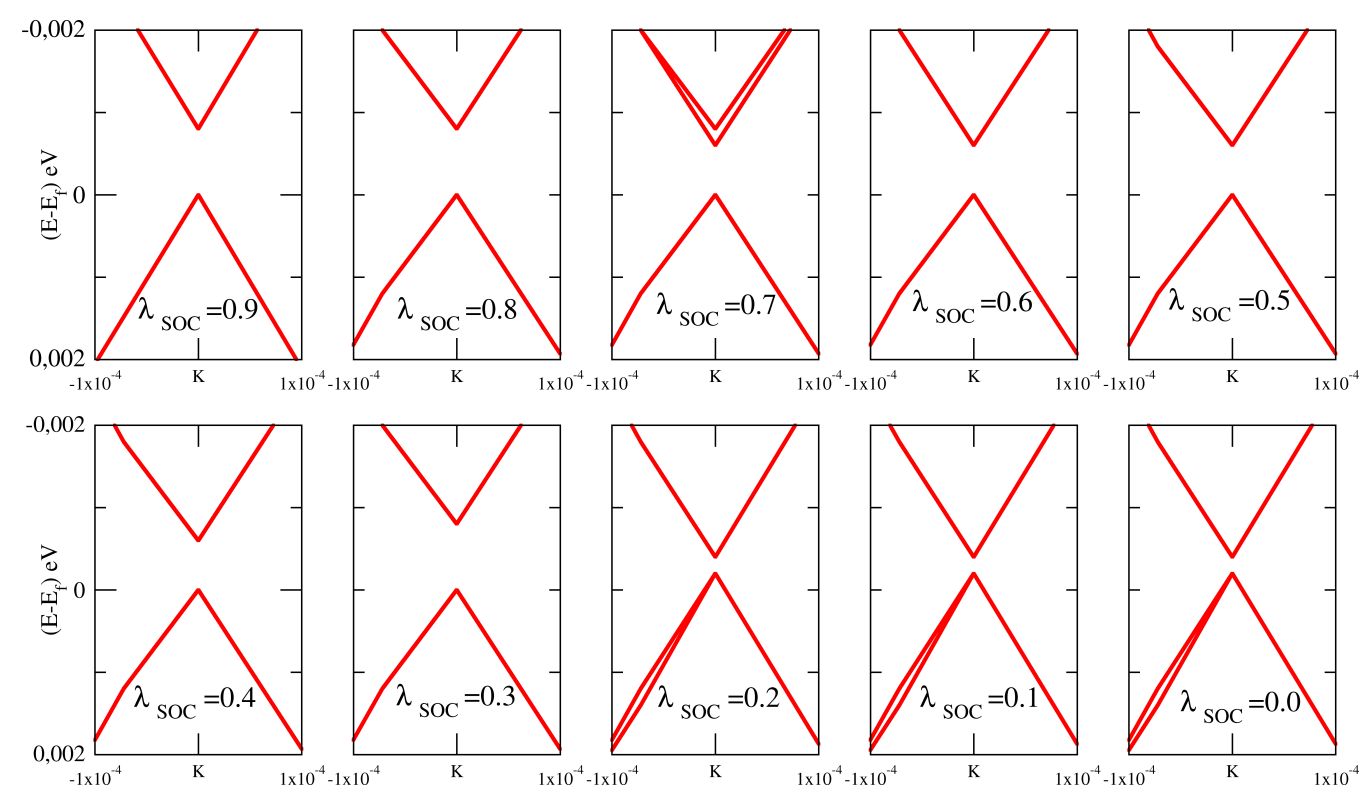

Figura 6.22: Mudança da estrutura de bandas do Ba adsorvido no grafeno em relação à variação da intensidade do SOC do átomo de Ba. 


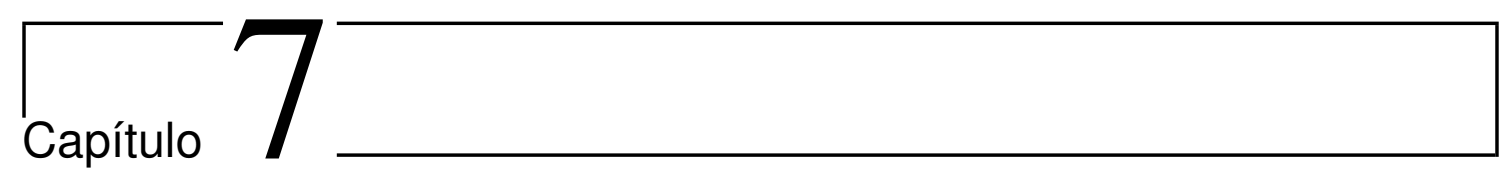

\section{Conclusões}

A partir do formalismo de pseudopotenciais relativísticos de norma conservada, foi implementada a aproximação "on-site" do SOC no código SIESTA. Para verificar o bom funcionamento desta implementação, foram estudados sistemas já conhecidos na literatura, que apresentam fortes perturbações associadas ao SOC. Os resultados obtidos concordam com os dados experimentais, e com outros códigos. Estes testes foram enfocados no estudo dos efeitos do SOC em átomos adsorvidos no grafeno, e de fases topológicas não-triviais em diferentes sistemas. Por exemplo, a formação de estados QSH no poço quântico de $\mathrm{HgTe} / \mathrm{CdTe}$ e a formação de estados metálicos protegidos pela simetria de reversão temporal na superfície do $\mathrm{Bi}_{2} \mathrm{Se}_{3}$. O estudo deste último fornece um resultado novo: Quando o Mn é adsorvido na superfície de $\mathrm{Bi}_{2} \mathrm{Se}_{3}$ com uma dopagem de $66 \%$ é possível a existência de ordem magnética (ferromagnética e antiferromagnética). A fase antiferromagnética é a mais estável, de modo que mesmo na presença de dopagem preserva-se a simetria de reversão temporal e o comportamento de férmions de Dirac sem massa é mantido.

A presente tese teve como foco principal o estudo de fases topológicas em uma rede hexagonal de baricentros. Mostramos que os estados do Ru quando adsorvidos no grafeno hibridizam fortemente com os estados dos carbonos vizinhos, 
de modo que este metal fixa as fases da função de onda de seus primeiros e segundos vizinhos. Quando os Ru são depositados numa configuração triangular e o centro geométrico dos triângulos de Ru coincide com a posição de um átomo de carbono, a propagação destas fases via grafeno resulta na interferência construtiva das funções de onda, gerando picos na densidade eletrônica nos centros dos triângulos formados pelos Ru. Como consequência é formada uma rede de "baricentros" e "antibaricentros" com simetria hexagonal. A estrutura de bandas do sistema apresenta uma dispersão tal que formam-se quatro cones de Dirac nos pontos $\mathrm{K}$ e K'. Foi mostrado que de forma efetiva os baricentros são responsáveis por esta particular estrutura eletrônica. Portanto, neste trabalho predizemos que a partir da deposição de átomos de Ru em uma configuração triangular acima do grafeno podese formar uma rede hexagonal de baricentros e antibaricentros que de forma efetiva geram quatro cones de Dirac nos pontos K e K' da primeira zona de Brillouin.

Mostramos que a singular estrutura de bandas do sistema de baricentros está associada com a formação de estados QHA. Adicionalmente, o forte SOC do Ru e o momento magnético resultante, caraterísticas do QHA, são necessários para a formação da rede de baricentros com simetria hexagonal. Consequentemente, a existência de baricentros que de forma efetiva geram o comportamento de férmions de Dirac sem massa são uma condição suficiente para a formação de estados topológicos associados com o efeito QHA. Entretanto, gerar uma fase QSH em um sistema de baricentros pela deposição de átomos pesados em grafeno é fisicamente contraditório. A partir de uma intensa procura determinou-se que o Ba e Sr quando adsorvidos no grafeno fornecem um momento magnético nulo de forma que preservam a simetria de reversão temporal, o qual resulta na estabilização de uma fase topológica associada com estados QSH. Os cones de Dirac nos pontos K e K’ do grafeno são perturbados somente pelo SOC destes materiais. A transferência de SOC dos metais ao grafeno resulta no aumento do gap de energia em mais de 1000 vezes. De forma que, a deposição de Ba e Sr em grafeno pode ser um caminho para possíveis aplicações do 
efeito QSH em spintrônica.

Neste trabalho foi mostrado que mediante a engenharia de superfícies podem-se gerar estruturas artificiais a partir do grafeno que apresentem efeitos do SOC maiores do que o sistema pristino, de forma a favorecer a existência de fases topológicas não triviais muito mais notórias, aumentando a possibilidade de aplicações práticas destas fases em spintrônica.

A possibilidade de induzir ou controlar as fases topológicas em materiais através da deposição de átomos é um tema de atual interesse. A implementação desenvolvida neste trabalho permite o estudo de sistemas compostos por muitos átomos, de forma que, o estudo da ordem magnética em superfícies de TI induzida pela deposição de metais é viável. Adicionalmente, é de fundamental interesse o estudo da interação spin-órbita em metais quando adsorvidos em sistemas com simetria hexagonal tal como o siliceno. O estudo de estados topológicos não-triviais e sua caraterização exige o cálculo do invariante topológico $Z_{2}$ e a inclusão do SOC no código de transporte. A implementação do SOC no código para o cálculo de transporte eletrônico TRANSAMPA está em andamento. 


\section{Bibliografia}

1. R. L. Laughlin, Phys. Rev. Lett. 50, 1395 (1983)

2. Thouless, Kohmoto, Nighting e Nijs, Phys. Rev. Lett. 49, 6 (1982)

3. C. L. Kane e E. J. Mele, Phys. Rev. Lett. 95, 226805 (2005)

4. H. Zhang et al., Nature Physics, 5 (2009)

5. M. König et al., Science 318, 766 (2007)

6. Hsieh et al., Nature 460, 1101 (2009)

7. M. Houssa et al., Appl. Phys. Lett. 98, 223107 (2011)

8. G. Dresselhaus Phys. Rev. 100, 580 (1955)

9. L. M. Roth, B. Lax, S. Zwerdling, Phys. Rev. 114, 90 (1959)

10. D. Malko et al., Phys. Rev. Lett. 108, 086804 (2012)

11. P.O Löwdin, Adv. Chem. Phys. 2, 207 (1959)

12. L. Thomas e E. Fermi, Proc. Camb. Phil. Soc. 23 (1927)

13. P. Hoenberg e W. Kohn, Phys. Rev. 136, B864 (1964)

14. W. Khan e L. Sham, Phys. Rev. 140, A1133 (1965)

15. J. C. Philips e L. Kleinman, Phys. Rev. 116, 257 (1959)

16. C. Hering, Phys. Rev. 57, 1169 (1940)

17. L. R. Kann, P. Baybuit e D. G. Trublar, J. Chem. Phys. 65, 3826 (1976)

18. L. R. Kahn e W. A. Goddard III, J. Chem. Phys. 56, 2685 (1972)

19. C. F. Mellus e W. A. Goddard III, Phys. Rev. A 10, 1528 (1974) 
20. P. A. Christiansen, Y. S. Lee e k. S. Pitzer, J. Chem. Phys. 71, 4445 (1979)

21. J. Callaway. Quantum Theory of the Solid States, student edition (1970)

22. A. Zunger e M. Cohen, Phys. Rev. Lett. 41, 53 (1978)

23. L. Kleinman, Phys. Rev. B 59, 3314 (1999)

24. L. Fernandez-Seivane et al., J. Phys.: Condens. Matter 18, 7999 (2006)

25. G. B Bachelet, D. R. Hamann e D. G Schlüter, Phys. Rev. B 26, 4199 (1982)

26. D. R. Hamman, M. Schluter e C. Chiang, Phys. Rev. Lett, 43, 194 (1979)

27. A. J. Silva Oliveira. Instituto de Física - UNICAMP- Tese de doutorado (1997)

28. Soler et al., J. Phys.: Condens. Matter 14 (2002)

29. G. G. Wepfer, T. C. Collins, e R. N. Euwema, Phys. Rev B, 4, 1296 (1971)

30. L. D. Landau e G. M. Lisfshitz, Statistical Physics UK (1980)

31. K. V. Klitzing, G. Dorda e M. Pepper, Phys. Rev. Lett 45, 6 (1980)

32. D. J. Thouless et al., Phys. Rev. Lett 49, 405 (1982)

33. M. C. Chang e Q. Niu, Phys. Rev. B 53, 7010 (2010)

34. Y. G. Yao et al., Phys. Rev. Lett 92, 037204 (2004)

35. S. Murakami, N. Nagaosa e S. C. Zhang, Science 301, 1348 (2003)

36. J. Sinova et al., Phys. Rev. Lett 92, 126603 (2004)

37. Y. K. Kato et al., Science 306, 1910 (2004)

38. J. Wunderlich et al., Condens. Matter, 0410295 (2004)

39. F. D. Haldane, Phys. Rev. Lett 93, 206602 (2004)

40. X. L. Qi, T. L. Hughes e S. C. Zhang, Phys. Rev. B 78, 195424 (2008)

41. B. A. Bernevig, T. L. Hughes, S. C. Zhang Science 314, 1757 (2006)

42. L. Fu e C. L. Kane, Phys. Rev. B 76, 045302 (2007)

43. H. Groves, R. N. Brown e C. R. Pidgeon, Phys. Rev 161, 779 (1967)

44. S. Chadov et al., Physico Status Solidi, 28 (2012)

45. J. W. Luo e A. Zunger. Phys. Rev. Lett 105, 176805 (2010) 
46. O. A. Pankratov, S. V. Pakhomov e B. A. Volkov, Sol. Stat. Comm 61, 2 (1987)

47. C. Liu et al., Phys. Rev. Lett 100, 236601 (2008)

48. L. Fu, C. L. Kane e E. J. Mele, Phys. Rev. Lett 98, 1066803 (2007)

49. J. E. Moore e L. Balents, Phys. Rev. B 75, 121306 (2007)

50. R. Roy, Phys. Rev. B 79, 195321 (2009)

51. Y. Yan, Y. Zhang e A. Vishwanath, Nature Physics 5, 298 (2009)

52. Y. L Chen et al., Science 239 (2010)

53. Y. S. Hor et al., Phys. Rev. B 81, 19503 (2010)

54. F. Kuemmeth et al., Nature 452, 7186 (2008)

55. Yao et al., Phys. ev. B 75, 4 (2007)

56. M. Gmitra et al., Phys. Rev. B 80, 23 (2009)

57. D. Huertas, F. Guinea, A. Brataas, Phys. Rev. B, 74, 15 (2006)

58. A . K. Geim, K. S. Novoselov, Nature Matter 6, 3 (2007)

59. A. H. Castro Neto et al., Rev. Mod. Phys 81, 1 (2009)

60. I. Zutic, J. Fabian e S. Das Sarma, Rev. Mod. Phys 76, 2 (2004)

61. D. D. Awschalom e M. E. Flatte, Nature Physics 3, 3 (2007)

62. Z. Zhang, C. Chen e W. Guo, Phys. Rev. Lett 103, 18 (2009)

63. Y. S. Dedkov et al., Phys. Rev. Lett 100, 10 (2008)

64. O. Rader et al., Phys. Rev. Lett 102, 057602 (2009)

65. Z. Y. Li et al., J. Phys. Condens Matter 23, 225502 (2011)

66. M. P. Lima, A. J. Da Silva e A. Fazzio, Phys. Rev. B 84, 245411 (2011)

67. K. Glantsching e C. Ambrosch, New J. Phy 12, 103048 (2010)

68. G. Kenjiro et al., Nature Letter 483, (2012)

69. C. Weeks et al., Phys. Rev. X 1, 021001 (2011)

70. J. Ding et al., Phys. Rev. B 84, 195444 (2011)

71. H. Min et al., Phys. Rev. B 74, 165310 (2006)

72. J. C. Boettger e S. B Trickey, Phy. Rev. B 75121402 (2007) 
73. F. Guinea et al., Phys. Rev. B 81, 035408 (2010)

74. D. Ma, Z. Li e Z. Yang, Carbon 50, 1 (2012)

75. Z. Qiao et al., Phys. Rev. B 82, 161414 (2010)

76. D. Xiao et al., Phys. Rev. Lett 99, 236809 (2007)

77. J. Maciejko, T. L. Hughes e S. C. Zhang, Annu. Rev. Condens. Matter Phys. 2, 31-53 (2011) 